\title{
The cyclopropane ring as a reporter of radical leaving-group reactivity for Ni-catalyzed $\mathbf{C}\left(\mathrm{sp}^{3}\right)-\mathrm{O}$ arylation
}

\author{
L. Reginald Mills, ${ }^{1}$ John J. Monteith, ${ }^{1}$ Gabriel dos Passos Gomes, ${ }^{1,2}$ Alán Aspuru-Guzik, $, 2,3,4$ \\ and Sophie A. L. Rousseaux ${ }^{1 *}$ \\ *sophie.rousseaux@utoronto.ca

\begin{abstract}
${ }^{1}$ Department of Chemistry, University of Toronto, 80 St. George Street, Toronto, ON M5S 3H6, Canada ${ }^{3}$ Department of Computer Science, University of Toronto, 214 College St., Toronto, Ontario M5T 3A1, Canada ${ }^{4}$ Vector Institute for Artificial Intelligence, 661 University Ave. Suite 710, Toronto, Ontario M5G 1M1, Canada ${ }^{5}$ Lebovic Fellow, Canadian Institute for Advanced Research (CIFAR), 661 University Ave, Toronto, ON M5G 1M1, Canada
\end{abstract}

\section{Supporting Information 1: Experimental Data}

\section{Table of Contents}

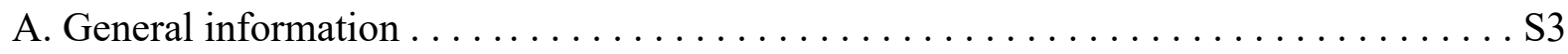

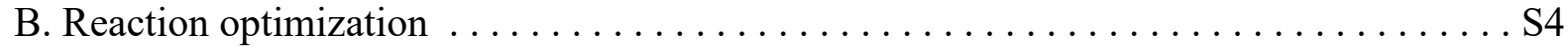

Table $\mathrm{S} 1$. Evaluation of cyclopropanol leaving group . . . . . . . . . . S4

Table S2. Evaluation of Ni sources and ligands . . . . . . . . . . S5

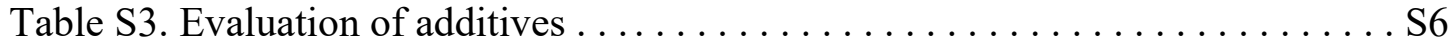

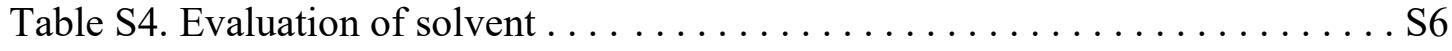

Table S5. Evaluation of arylzinc stoichiometry $\ldots \ldots \ldots \ldots \ldots \ldots \ldots \ldots \ldots \ldots$

Table S6. Evaluation of arylzinc reagent . . . . . . . . . . . . . S8

Table S7. Ring-opening of 1-phenylcyclopropyl tosylate $\ldots \ldots \ldots \ldots \ldots \ldots$ S8

C. Preparation of cyclopropane products $\ldots \ldots \ldots \ldots \ldots \ldots \ldots \ldots \ldots \ldots \ldots$

General Procedure A: Synthesis of cyclopropanes ... . . . . . . . . . S9

General Procedure B: Synthesis of cyclopropanes from thiobenzoates . . . . . . . S9

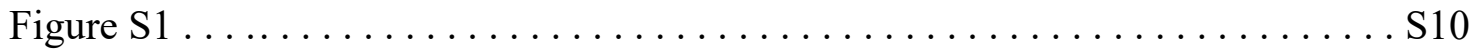

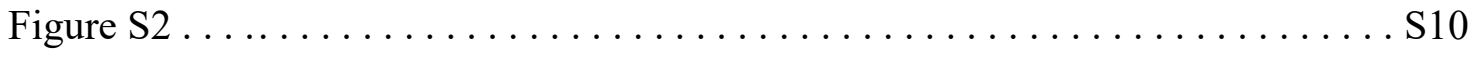


Table S8. Complete scope table .............................. S11

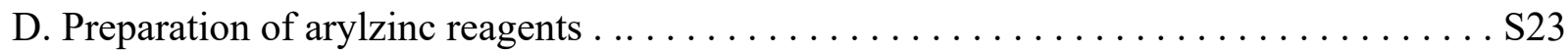

D.1. Procedure for preparation of arylzinc reagents . . . . . . . . . . . . S23

General Procedure C: Preparation of arylzinc reagents ............... S23

Table S9. Scale and concentration of arylzinc reagents prepared for this study . . . S24

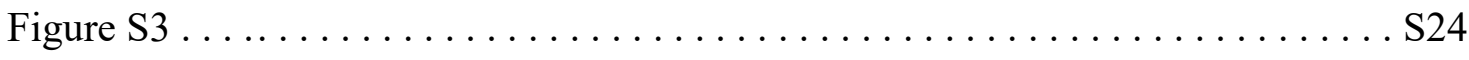

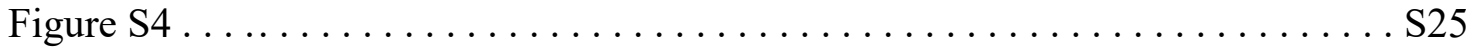

D.2. Preparation of aryl bromide starting materials . . . . . . . . . . . S26

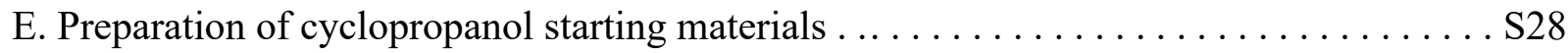

E.1. Preparation of cyclopropanols with alternate leaving groups . . . . . . . . S28

E.2. Preparation of standard thiocarbamate substrates . . . . . . . . . . . . S33

General Procedure D: Preparation of thiocarbamate starting materials . . . . . . . . S33

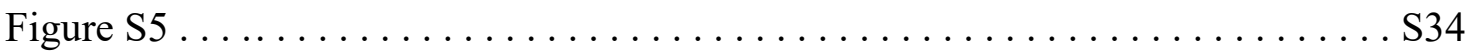

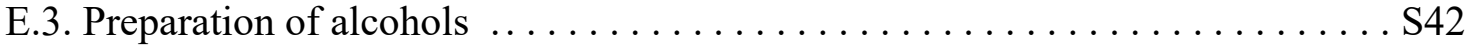

General Procedure E: Simmons-Smith sequence . .................. S42

E.4. Preparation of thiobenzoates . . . . . . . . . . . . . . . . . . . S 48

F. Mechanistic experiments..................................... S49

F.1. Barton-McCombie deoxygenation of $1 \mathbf{k} \ldots \ldots \ldots \ldots \ldots \ldots \ldots \ldots$. . . . . . . 49

Equation S1. Barton-McCombie deoxygenation of $1 \mathbf{k} \ldots \ldots \ldots \ldots \ldots \ldots$. . . . . . 49

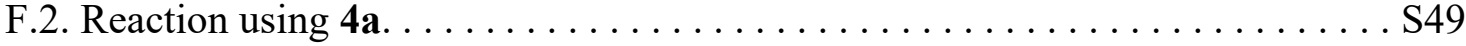

F.3. Crossover experiments $\ldots \ldots \ldots \ldots \ldots \ldots \ldots \ldots \ldots \ldots \ldots \ldots \ldots$. . . . . . . . . . . 49

F.4. Loss of enantiomeric excess . . . . . . . . . . . . . . . . . S51

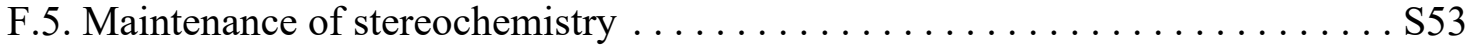

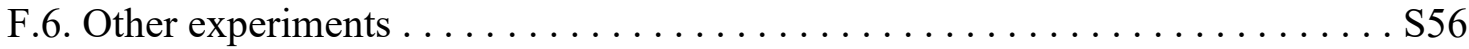

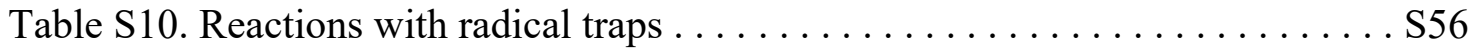

Table S11. Yield of cyclopropane and thioester with other leaving groups $\ldots . \ldots$ S56

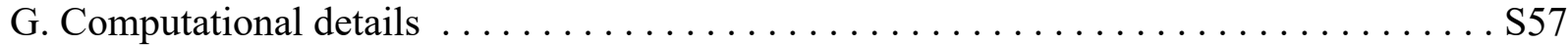

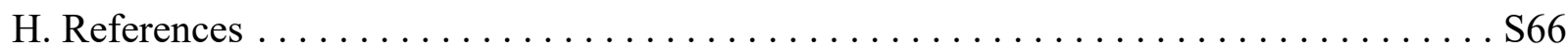




\section{A. General information}

Unless otherwise noted, all reactions were set up on the benchtop and run under an atmosphere of Ar or $\mathrm{N}_{2}$ using flame-dried glassware and anhydrous solvents. DCM, $\mathrm{Et}_{2} \mathrm{O}, \mathrm{MeCN}, \mathrm{PhMe}$, and THF were purchased as HPLC-grade (inhibitor-free) from Caledon or Sigma-Aldrich, and were dried using a PureSolv MD 5 solvent purification system and used without further manipulation. 1,4-Dioxane was purchased as anhydrous from Sigma-Aldrich in a Sure/Seal bottle and was degassed by sonicating under vacuum for $15 \mathrm{~min}$ prior to use. $\mathrm{Ni}(\mathrm{acac})_{2} \bullet \mathrm{xH}_{2} \mathrm{O}$ was purchased from Sigma-Aldrich as $\mathrm{Ni}(\mathrm{acac})_{2}$ and was stored in a dessicator open to air, so was assumed to have a small amount of hydration and was denoted as " $\mathrm{Ni}(\text { acac })_{2} \bullet \mathrm{xH}_{2} \mathrm{O}$ ". $\mathrm{Ni}(\mathrm{acac})_{2}$ was purchased from Strem and was stored in a glovebox. $\mathrm{Mg}(\mathrm{II})$ and $\mathrm{Zn}$ (II) salts were purchased as anhydrous-grade and were stored and weighed in a glovebox. $\mathrm{Zn}(\mathrm{OMe})_{2}$ for the formation of arylzinc reagents was prepared in situ from LiOMe and $\mathrm{ZnCl}_{2}$ by stirring for $1 \mathrm{~h}$ in THF (see Section D). ${ }^{1}$ All other commercial reagents were used as received. Compounds were purified by flash column chromatography using SiliCycle SilicaFlash P60 silica gel. The 8- and 16-mL threaded culture tubes used for reactions were purchased from Fisher (catalogue nos. 14-95776A and 14-959-35A) and were sealed using size 19 rubber septa and electrical tape.

Thiocarbamate starting materials were stored at r.t. on the benchtop with no special precautions for air, moisture, or light. Arylzinc reagents were stored in culture tubes under $\mathrm{N}_{2}$ at r.t. Electronrich cyclopropanes are known to be unstable to air. ${ }^{2}$ As a precaution, all crude residues were stored under $\mathrm{N}_{2}$ at $-20{ }^{\circ} \mathrm{C}$, and all electron-rich cyclopropane products were kept under $\mathrm{N}_{2}$ at $-20^{\circ} \mathrm{C}$ for long-term storage.

GC-MS data was obtained on a Shimadzu GCMS-QP2010 SE; yields represent peak areas calibrated against each compound's response factor relative to $n$-dodecane internal standard. ${ }^{1} \mathrm{H}$ and ${ }^{13} \mathrm{C}$ NMR spectra were recorded on Varian MercuryPlus $400 \mathrm{MHz}$, Agilent DD2 $500 \mathrm{MHz}$, or Bruker AvanceIII $400 \mathrm{MHz}$ spectrometers. TLC samples were run on EMD Millipore TLC Silica gel $60 \mathrm{~F}_{254}$ plates and were visualized by UV or by staining with standard $\mathrm{KMnO}_{4}$, phosphomolybdic acid (PMA), $p$-anisaldehyde, or vanillin stains. IR spectra were obtained on a Perkin-Elmer Spectrum 100 instrument equipped with a single-bounce diamond/ZnSe ATR accessory as solids or thin films. Melting points were obtained on a Fisher-Johns Melting Point Apparatus. High-resolution mass spectra (HRMS) were recorded on a JEOL AccuTOF JMST1000LV mass spectrometer equipped with a Direct Analysis in Real Time (DART) ion source. Chiral HPLC analysis was performed on a Shimadzu 20A series system using a Daicel Chiralpak column (IA).

The following non-standard abbreviation is used in this SI:

"DCM" $=\mathrm{CH}_{2} \mathrm{Cl}_{2}$, dichloromethane. 


\section{B. Reaction optimization}

Table S1. Evaluation of cyclopropanol leaving group

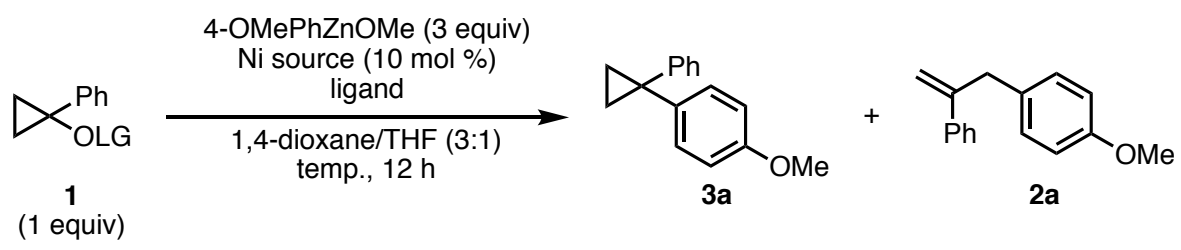

\begin{tabular}{|c|c|c|c|c|c|c|c|}
\hline Entry & -OLG (leaving group) & & Ni source & ligand (mol \%) & $\begin{array}{c}\text { Temp } \\
\left({ }^{\circ} \mathrm{C}\right)\end{array}$ & $\begin{array}{c}\text { Yield } \\
\mathbf{3 a} \\
(\%)^{a}\end{array}$ & $\begin{array}{c}\text { Yield } \\
\mathbf{2 a} \\
(\%)^{a}\end{array}$ \\
\hline 1 & & \multirow{3}{*}{ (1a) } & $\mathrm{Ni}(\text { acac })_{2} \cdot \times \mathrm{H}_{2} \mathrm{O}$ & bathocuproine (20) & 23 & 0 & 9 \\
\hline 2 & & & $\mathrm{NiCl}_{2}\left(\mathrm{PPh}_{3}\right)_{2}$ & none & 110 & 0 & 96 \\
\hline 3 & & & $\mathrm{NiCl}_{2}\left(\mathrm{PCy}_{3}\right)_{2}$ & none & 110 & 0 & 41 \\
\hline 4 & & \multirow{3}{*}{ (1b) } & $\mathrm{Ni}(\mathrm{acac})_{2} \times \mathrm{H}_{2} \mathrm{O}$ & bathocuproine (20) & 23 & 0 & 3 \\
\hline 6 & & & $\mathrm{NiCl}_{2}\left(\mathrm{PPh}_{3}\right)_{2}$ & none & 110 & 0 & 96 \\
\hline 6 & & & $\mathrm{NiCl}_{2}\left(\mathrm{PCy}_{3}\right)_{2}$ & none & 110 & 0 & 52 \\
\hline 7 & & \multirow{2}{*}{ (S6) } & $\mathrm{Ni}(\mathrm{acac})_{2} \times \mathrm{H}_{2} \mathrm{O}$ & bathocuproine (20) & 23 & 0 & 0 \\
\hline 8 & & & $\mathrm{NiCl}_{2}\left(\mathrm{PCy}_{3}\right)_{2}$ & none & 110 & 0 & 0 \\
\hline 9 & & \multirow{2}{*}{ (1d) } & $\mathrm{Ni}(\mathrm{acac})_{2} \times \mathrm{H}_{2} \mathrm{O}$ & bathocuproine $(20)$ & 23 & 0 & 0 \\
\hline 10 & & & $\mathrm{NiCl}_{2}\left(\mathrm{PCy}_{3}\right)_{2}$ & none & 110 & 0 & trace \\
\hline 11 & & \multirow{2}{*}{ (1c) } & $\mathrm{Ni}(\mathrm{acac})_{2} \times \mathrm{H}_{2} \mathrm{O}$ & bathocuproine (20) & 23 & 0 & 0 \\
\hline 12 & & & $\mathrm{NiCl}_{2}\left(\mathrm{PCy}_{3}\right)_{2}$ & none & 110 & 0 & 3 \\
\hline 13 & ? & \multirow{3}{*}{ (1f) } & $\mathrm{Ni}(\mathrm{acac})_{2} \times \mathrm{H}_{2} \mathrm{O}$ & bathocuproine (20) & 23 & 0 & 0 \\
\hline 14 & & & $\mathrm{NiCl}_{2}\left(\mathrm{PPh}_{3}\right)_{2}$ & none & 110 & 0 & trace \\
\hline 15 & & & $\mathrm{NiCl}_{2}\left(\mathrm{PCy}_{3}\right)_{2}$ & none & 110 & 1 & 0.6 \\
\hline 16 & & \multirow{4}{*}{ (1g) } & $\mathrm{Ni}(\mathrm{acac})_{2} \times \mathrm{H}_{2} \mathrm{O}$ & bathocuproine (20) & 23 & 0 & 0 \\
\hline 17 & & & $\mathrm{NiCl}_{2}\left(\mathrm{PCy}_{3}\right)_{2}$ & none & 23 & 0.3 & 0.2 \\
\hline 18 & & & $\mathrm{NiCl}_{2}\left(\mathrm{PPh}_{3}\right)_{2}$ & none & 110 & trace & 0 \\
\hline 19 & & & $\mathrm{NiCl}_{2}\left(\mathrm{PCy}_{3}\right)_{2}$ & none & 110 & 1 & 0.4 \\
\hline 20 & & \multirow{3}{*}{ (1e) } & $\mathrm{Ni}(\mathrm{acac})_{2} \times \mathrm{H}_{2} \mathrm{O}$ & bathocuproine (20) & 23 & 0 & 0 \\
\hline $21^{b}$ & & & $\mathrm{NiCl}_{2}(\mathrm{dme})$ & dtbbpy (20) & 23 & 1 & 0 \\
\hline 22 & & & $\mathrm{NiCl}_{2}\left(\mathrm{PPh}_{3}\right)_{2}$ & none & 110 & 0 & 0 \\
\hline 23 & & \multirow{2}{*}{ (1i) } & $\mathrm{Ni}(\mathrm{acac})_{2} \cdot \mathrm{xH}_{2} \mathrm{O}$ & bathocuproine $(20)$ & 23 & 29 & 3 \\
\hline 24 & & & $\mathrm{NiCl}_{2}\left(\mathrm{PCy}_{3}\right)_{2}$ & none & 110 & 14 & 10 \\
\hline 25 & & \multirow{3}{*}{$(1 \mathrm{j})$} & $\mathrm{Ni}(\mathrm{acac})_{2} \times \mathrm{H}_{2} \mathrm{O}$ & bathocuproine (20) & 23 & 54 & 0 \\
\hline 26 & & & $\mathrm{NiCl}_{2}\left(\mathrm{PPh}_{3}\right)_{2}$ & none & 110 & 40 & 6 \\
\hline 27 & & & $\mathrm{NiCl}_{2}\left(\mathrm{PCy}_{3}\right)_{2}$ & none & 110 & 37 & 5 \\
\hline 28 & & \multirow{6}{*}{ (1h) } & $\mathrm{Ni}(\mathrm{acac})_{2} \cdot \mathrm{xH}_{2} \mathrm{O}$ & bathocuproine (20) & 23 & 0 & 0 \\
\hline 29 & S & & $\mathrm{NiCl}_{2}(\mathrm{dme})$ & bathocuproine (20) & 23 & 0.5 & 0 \\
\hline 30 & & & $\mathrm{NiCl}_{2}(\mathrm{dme})$ & Bphen (20) & 23 & 0.6 & 0 \\
\hline 31 & & & $\mathrm{NiCl}_{2}(\mathrm{dme})$ & neocuproine (20) & 23 & 1 & 0 \\
\hline 32 & $\mathrm{Me}$ & & $\mathrm{NiCl}_{2}\left(\mathrm{PCy}_{3}\right)_{2}$ & none & 23 & 1 & 2 \\
\hline 33 & & & $\mathrm{NiCl}_{2}\left(\mathrm{PPh}_{3}\right)_{2}$ & none & 110 & 0 & trace \\
\hline 34 & & \multirow[b]{2}{*}{ (1k) } & $\mathrm{Ni}(\mathrm{acac})_{2} \cdot \mathrm{xH}_{2} \mathrm{O}$ & bathocuproine (20) & 23 & 77 & 0 \\
\hline 35 & $\mathrm{Bz}$ & & $\mathrm{NiCl}_{2}\left(\mathrm{PPh}_{3}\right)_{2}$ & none & 110 & 2 & 0 \\
\hline
\end{tabular}

aGC-MS yield using $n$-dodecane as internal standard; bUsing Ru(bpy) ${ }_{3}\left(\mathrm{PF}_{6}\right)_{2}(1 \mathrm{~mol} \%)$, blue LEDs, $\mathrm{ArZnCl}$ ( 2 equiv) and acetone/THF (1:1). 
Table S2. Evaluation of Ni sources and ligands

\begin{tabular}{|c|c|c|c|c|c|c|}
\hline & 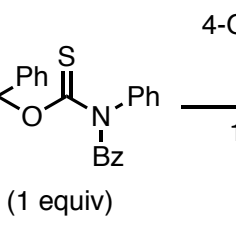 & $\begin{array}{l}\text { MePhZnOMe (3 equiv) } \\
\text { Ni source, ligand } \\
\text { additive }\end{array}$ & 3a & $4 a$ & $B=$ & $3 a^{\prime}$ \\
\hline Entry & Ni source & Ligand (mol \%) & Additive (equiv) & Yield 3a (\%) & Yield 4a (\%) & Yield 3a' (\%) \\
\hline 1 & $\mathrm{Ni}(\text { acac })_{2} \bullet \mathrm{xH}_{2} \mathrm{O}$ & bathocuproine (20) & none & 78 & 12 & 13 \\
\hline 2 & $\mathrm{Ni}(\mathrm{acac})_{2} \cdot \mathrm{xH}_{2} \mathrm{O}$ & bpy (20) & $\mathrm{MgBr}_{2}(2)$ & $<1$ & 29 & 43 \\
\hline 3 & $\mathrm{Ni}(\text { acac })_{2} \cdot \mathrm{xH}_{2} \mathrm{O}$ & dtbbpy (20) & $\mathrm{MgBr}_{2}(2)$ & 2 & 45 & 41 \\
\hline 4 & $\mathrm{Ni}(\mathrm{acac})_{2} \cdot \mathrm{xH}_{2} \mathrm{O}$ & phen (20) & $\mathrm{MgBr}_{2}(2)$ & 0 & 7 & 71 \\
\hline 5 & $\mathrm{Ni}(\text { acac })_{2} \cdot x_{2} \mathrm{O}$ & Bphen (20) & $\mathrm{MgBr}_{2}(2)$ & 0 & 21 & 49 \\
\hline 6 & $\mathrm{Ni}(\mathrm{acac})_{2} \cdot \mathrm{xH}_{2} \mathrm{O}$ & 2,9-dmphen (20) & $\mathrm{MgBr}_{2}(2)$ & 3 & 40 & 23 \\
\hline 7 & $\mathrm{Ni}(\mathrm{acac})_{2} \cdot \mathrm{xH}_{2} \mathrm{O}$ & tmphen (20) & $\mathrm{MgBr}_{2}(2)$ & $<1$ & 22 & 14 \\
\hline 8 & $\mathrm{Ni}(\text { acac })_{2} \cdot \mathrm{xH}_{2} \mathrm{O}$ & $\mathrm{Bn}-\mathrm{BIOX}(20)$ & $\mathrm{MgBr}_{2}(2)$ & $<1$ & $<1$ & 61 \\
\hline 9 & $\mathrm{Ni}(\text { acac })_{2} \cdot \mathrm{xH}_{2} \mathrm{O}$ & terpy (20) & $\mathrm{MgBr}_{2}(2)$ & $<1$ & 0 & 5 \\
\hline 10 & $\mathrm{Ni}(\text { acac })_{2} \cdot x_{2} \mathrm{O}$ & bpp (20) & $\mathrm{MgBr}_{2}(2)$ & 1 & 0 & 36 \\
\hline 11 & $\mathrm{Ni}(\text { acac })_{2} \cdot \mathrm{xH}_{2} \mathrm{O}$ & L1 (20) & $\mathrm{MgBr}_{2}(2)$ & 10 & 55 & 17 \\
\hline 12 & $\mathrm{Ni}(\mathrm{acac})_{2} \cdot \mathrm{xH}_{2} \mathrm{O}$ & L2 (20) & $\mathrm{MgBr}_{2}(2)$ & $<1$ & 41 & 10 \\
\hline 13 & $\mathrm{Ni}(\mathrm{acac})_{2}$ & none & none & 2 & 0 & 44 \\
\hline 14 & $\mathrm{Ni}(\text { acac })_{2}$ & bathocuproine (20) & none & 46 & 21 & 9 \\
\hline 15 & $\mathrm{Ni}(\mathrm{acac})_{2}$ & bathocuproine (15) & none & 42 & 26 & 10 \\
\hline 16 & $\mathrm{Ni}(\text { acac })_{2}$ & bathocuproine (12) & none & 37 & 45 & 8 \\
\hline 17 & $\mathrm{Ni}(\text { acac })_{2}$ & bathocuproine (10) & none & 33 & 38 & 14 \\
\hline 18 & $\mathrm{NiCl}_{2}(\mathrm{dme})$ & bathocuproine (20) & none & 51 & 17 & 9 \\
\hline 19 & $\mathrm{NiBr}_{2}(\mathrm{dme})$ & bathocuproine (20) & none & 39 & 38 & 11 \\
\hline 20 & $\mathrm{Ni}(\mathrm{OAc})_{2} \cdot 4 \mathrm{H}_{2} \mathrm{O}$ & bathocuproine (20) & none & 14 & 29 & 27 \\
\hline 21 & $\mathrm{NiCl}_{2} \cdot 6 \mathrm{H}_{2} \mathrm{O}$ & bathocuproine (20) & none & 29 & 50 & 11 \\
\hline 22 & $\mathrm{Ni}(\mathrm{dpm})_{2}$ & bathocuproine (20) & none & 14 & 2 & 37 \\
\hline 23 & $\mathrm{Ni}(\mathrm{acac})_{2} \cdot \mathrm{xH}_{2} \mathrm{O}$ & $\mathrm{SIPr} \cdot \mathrm{HCl}(12)$ & none & 1 & 0 & 27 \\
\hline 24 & $\mathrm{Ni}(\text { acac })_{2} \cdot \mathrm{xH}_{2} \mathrm{O}$ & $\mathrm{IPr} \cdot \mathrm{HCl}(12)$ & none & 1 & 0 & 32 \\
\hline 25 & $\mathrm{Ni}(\text { acac })_{2} \cdot \mathrm{xH}_{2} \mathrm{O}$ & IMes•HCl (12) & none & 1 & 2 & 27 \\
\hline 26 & $\mathrm{Ni}(\text { acac })_{2} \cdot x_{2} \mathrm{O}$ & dppf (12) & none & 0 & 3 & 5 \\
\hline 27 & $\mathrm{Ni}(\text { acac })_{2} \cdot \mathrm{xH}_{2} \mathrm{O}$ & CyJohnPhos (12) & none & 1 & 0 & 17 \\
\hline 28 & $\mathrm{NiCl}_{2}\left(\mathrm{PPh}_{3}\right)_{2}$ & none & none & 0 & 0 & 3 \\
\hline 29 & $\mathrm{NiCl}_{2}\left(\mathrm{PC}_{3}\right)_{2}$ & none & none & $<1$ & 0 & 15 \\
\hline 30 & $\mathrm{NiCl}_{2}$ (dppe) & none & none & 0 & 0 & 0 \\
\hline 31 & none & none & none & 0 & 0 & 0 \\
\hline
\end{tabular}

GC-MS yields using $n$-dodecane as internal standard.

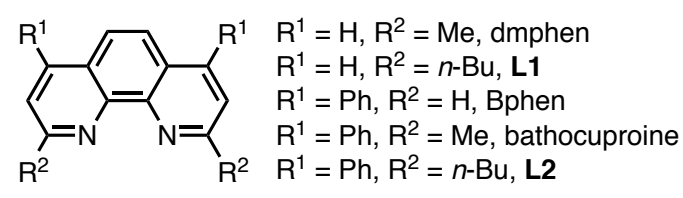<smiles>Cc1cnc2c(ccc3c(C)c(C)cnc32)c1C</smiles> 
Table S3. Evaluation of additives

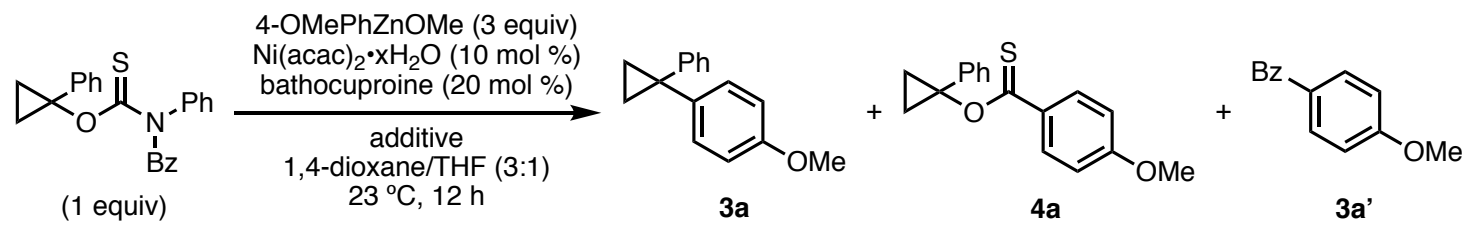

\begin{tabular}{|c|c|c|c|c|}
\hline Entry & Additive & Yield 3a (\%) & Yield 4a (\%) & Yield 3a' (\%) \\
\hline 1 & none & 77 & 12 & 13 \\
\hline 2 & $\mathrm{MgCl}_{2}(2)$ & 73 & 12 & 5 \\
\hline 3 & $\mathrm{MgBr}_{2}(2)$ & 61 & 14 & 5 \\
\hline 4 & $\mathrm{MgBr}_{2}(3)$ & 57 & 7 & 12 \\
\hline 5 & $\mathrm{Mgl}_{2}(1)$ & 0 & 0 & 0 \\
\hline 6 & $\mathrm{LiCl}(1)$ & 50 & 9 & 12 \\
\hline 7 & $\operatorname{LiBr}(1)$ & 50 & 10 & 11 \\
\hline 8 & LiOAc (1) & 47 & 5 & 12 \\
\hline 9 & $\mathrm{ZnCl}_{2}(1)$ & 2 & 0 & 36 \\
\hline 10 & $\mathrm{ZnBr}_{2}(1)$ & 3 & 0 & 59 \\
\hline 11 & $\mathrm{Znl}_{2}(1)$ & 2 & 0 & 50 \\
\hline 12 & $\mathrm{Zn}(\mathrm{OAc})_{2}(1)$ & 13 & 26 & 26 \\
\hline 13 & $\mathrm{Zn}(\mathrm{OMe})_{2}(1)$ & 30 & 0 & 14 \\
\hline 14 & $\mathrm{ZnCl}_{2}(1)$ and $\mathrm{MgBr}_{2}$ (1) & 5 & 0 & 51 \\
\hline
\end{tabular}

GC-MS yields using $n$-dodecane as an internal standard.

Table S4. Evaluation of solvent

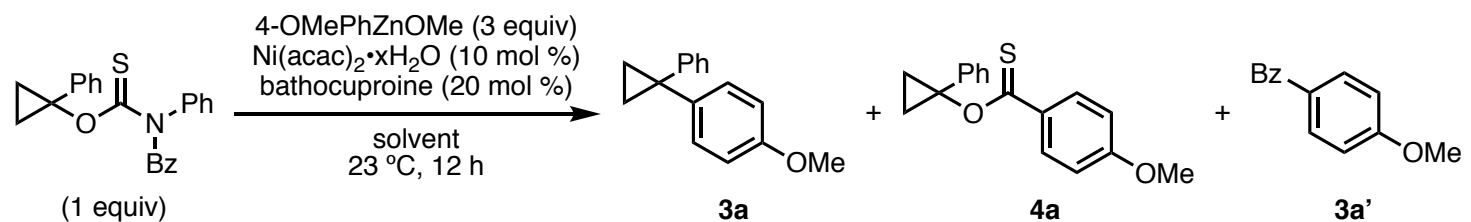

\begin{tabular}{ccccc}
\hline Entry & solvent & Yield 3a (\%) & Yield 4a (\%) & Yield 3a' (\%) \\
\hline 1 & 1,4-dioxane/THF (3:1) & 77 & 12 & 13 \\
2 & PhMe/THF (3:1) & 21 & 16 & 36 \\
3 & THF & 1 & 66 & 34 \\
4 & DME/THF (3:1) & 3 & 17 & 49 \\
5 & CMPE/THF (3:1) & 0 & 0 & 0 \\
6 & MTBE/THF (3:1) & 36 & 24 & 21 \\
7 & DMF/THF (3:1) & 0 & 0 & 0 \\
8 & MeCN/THF (3:1) & 0 & 34 & 16 \\
9 & DCM/THF (3:1) & 2 & 24 & 7 \\
10 & Et ${ }_{2}$ O/THF (3:1) & 1 & & \\
\hline
\end{tabular}

GC-MS yields using $n$-dodecane as an internal standard. 
Table S5. Evaluation of arylzinc stoichiometry

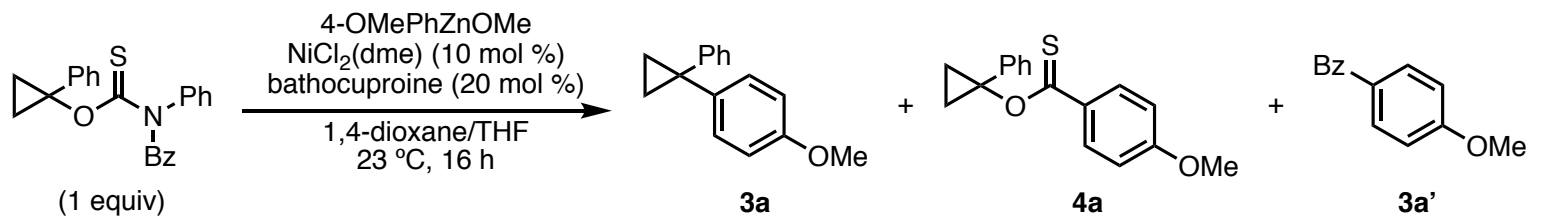

\begin{tabular}{cccccc}
\hline Entry & Ratio 1,4-dioxane/THF & equiv ArZnOMe & Yield 3a $(\%)^{b}$ & ${\text { Yield 4a }(\%)^{b}}$ & ${\text { Yield } \mathbf{3 a} \mathbf{a}^{\prime}(\%)^{b}}^{b}$ \\
\hline 1 & $2.9: 1$ & 1.4 & 1 & 71 & 8 \\
2 & $2.5: 1$ & 1.6 & 1 & 90 & 10 \\
3 & $1.0: 1$ & 2.3 & 6 & 79 & 15 \\
4 & $1.8: 1$ & 2.3 & 9 & 50 & 18 \\
5 & $1.9: 1$ & 2.3 & 10 & 53 & 18 \\
6 & $3.0: 1$ & 2.3 & 32 & 40 & 13 \\
7 & $4.0: 1$ & 2.3 & 36 & 36 & 14 \\
8 & $1.3: 1$ & 3.0 & 37 & $<5$ & 13 \\
9 & $1.5: 1$ & 3.0 & 40 & $<5$ & 14 \\
10 & $2.0: 1$ & 3.0 & 41 & 0 & 12 \\
11 & $2.7: 1$ & 3.0 & 44 & 0 & 9 \\
12 & $3.0: 1$ & 3.0 & 51 & 17 & 13 \\
13 & $4.0: 1$ & 3.0 & 47 & $<5$ & 17 \\
14 & $1.2: 1$ & 3.4 & 38 & 0 & 14 \\
15 & $1.0: 1$ & 4.0 & 33 & 0 & 28 \\
16 & $2.0: 1$ & 4.0 & 43 & 0 & 0 \\
17 & $0.9: 1$ & 4.4 & 37 & 0 & 17 \\
\hline
\end{tabular}

The reaction concentration depended on the concentration of arylzinc, which was generally $0.30-0.40 \mathrm{M}$ in THF. ${ }^{b}$ GC-MS yields using $n$-dodecane as an internal standard. 
Table S6. Evaluation of arylzinc reagents

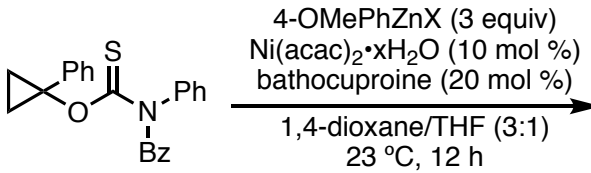

(1 equiv)

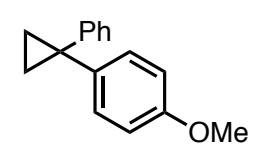

3a<smiles>COc1ccc(C(=S)OC2([PH3+])C[C@@H]2C)cc1</smiles>

$4 a$<smiles>COc1ccc(Br)cc1</smiles>

3a'

\begin{tabular}{|c|c|c|c|c|}
\hline Entry & $-\mathrm{Zn} \mathrm{X}^{a}$ & Yield 3a $(\%)^{b}$ & Yield 4a $(\%)^{b}$ & Yield 3a' (\%) \\
\hline 1 & $-\mathrm{ZnOMe} \cdot \mathrm{MgX}_{2} \mathrm{c}$ & 77 & 12 & 13 \\
\hline 2 & $-\mathrm{ZnCl} \cdot \mathrm{MgX}_{2}$ & 68 & 5 & 11 \\
\hline 3 & $-\mathrm{ZnBr} \cdot \mathrm{MgX} \mathrm{X}_{2}$ & 61 & 0 & 8 \\
\hline 4 & $-\mathrm{Znl} \cdot \mathrm{MgX}_{2}$ & 65 & 0 & 7 \\
\hline 5 & $-\mathrm{ZnOAc} \cdot \mathrm{MgX}_{2}{ }^{d}$ & 0 & 0 & 0 \\
\hline 6 & $\mathrm{Ar}_{2} \mathrm{Zn}$ (1.5 equiv) & 35 & 2 & 15 \\
\hline 7 & $\mathrm{Ar}_{2} \mathrm{Zn}$ (3 equiv) & 7 & 0 & 10 \\
\hline 8 & $-\mathrm{ZnCl} \cdot \mathrm{LiCl}$ & 57 & 2 & 9 \\
\hline 9 & $-\mathrm{ZnOMe} \cdot \mathrm{LiCle}$ & trace & 0 & 7 \\
\hline $10^{f}$ & -ZnOMe•LiCle & trace & 31 & 3 \\
\hline 11 & $-\mathrm{MgBr}^{g}$ & 0 & 0 & 8 \\
\hline 12 & $-\mathrm{ZnO} t-\mathrm{Bu} \cdot \mathrm{MgX} \mathrm{X}_{2}$ & 3 & 81 & 8 \\
\hline 13 & $-\mathrm{ZnOPh} \cdot \mathrm{MgX}_{2}$ & 0 & 0 & 0 \\
\hline 14 & $-\mathrm{ZnSPh} \cdot \mathrm{MgX} \mathrm{X}_{2}$ & 0 & 0 & 0 \\
\hline
\end{tabular}

aFor arylzinc reagents denoted as $-\mathrm{ZnX} \cdot \mathrm{MgX} \mathrm{X}_{2}$, the reagent was prepared from the Grignard (ArMgBr$\left.\cdot \mathrm{LiCl}\right)$, which was made from 4-bromoanisole (1 equiv), $\mathrm{Mg}(0)$ turnings (2 equiv), and $\mathrm{LiCl}$ (1.2 equiv). For arylzinc reagents denoted as $-\mathrm{ZnX} \cdot \mathrm{LiCl}$, the reagent was prepared from the aryllithium, which was made from 4-bromoanisole (1 equiv) and $n$-BuLi (1.1 equiv) via lithium-halogen exchange.

${ }^{b} \mathrm{GC}-\mathrm{MS}$ yields using $n$-dodecane as an internal standard.

cUsing $\mathrm{Zn}(\mathrm{OMe})_{2}$ prepared in situ from $\mathrm{ZnCl}_{2}$ (1 equiv) and $\mathrm{LiOMe} \mathrm{(2} \mathrm{equiv).}$

dThe arylzinc solution was heterogenerous.

eUsing $\mathrm{Zn}(\mathrm{OMe})_{2}$ prepared from $\mathrm{MeOH}$ and $\mathrm{Et}_{2} \mathrm{Zn}$.

$\mathrm{MgCl}_{2}$ (2 equiv) was added to the reaction.

$g$ Using the corresponding Grignard reagent rather than the arylzinc reagent.

Table S7. Evaluation of arylzinc reagents for ring-opening of 1-phenylcyclopropyl tosylate

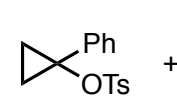

(1 equiv)<smiles></smiles>

(3 equiv)

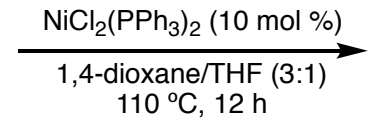

$110^{\circ} \mathrm{C}, 12 \mathrm{~h}$<smiles>C=C(Cc1ccc(OC)cc1)c1ccccc1</smiles>

$2 a$

\begin{tabular}{ccc}
\hline Entry & $-\mathrm{ZnX}$ & ${\text { Yield 2a }(\%)^{b}}^{b}$ \\
2 & $-\mathrm{ZnOMe} \cdot \mathrm{MgX}_{2}$ & 96 \\
3 & $-\mathrm{ZnCl} \cdot \mathrm{MgX}{ }_{2}$ & 63 \\
4 & $-\mathrm{ZnCl} \cdot \mathrm{LiX}$ & 11 \\
\hline
\end{tabular}

GC-MS yields using $n$-dodecane as internal standard. 


\section{Preparation of cyclopropane products}

General Procedure A: Synthesis of cyclopropanes

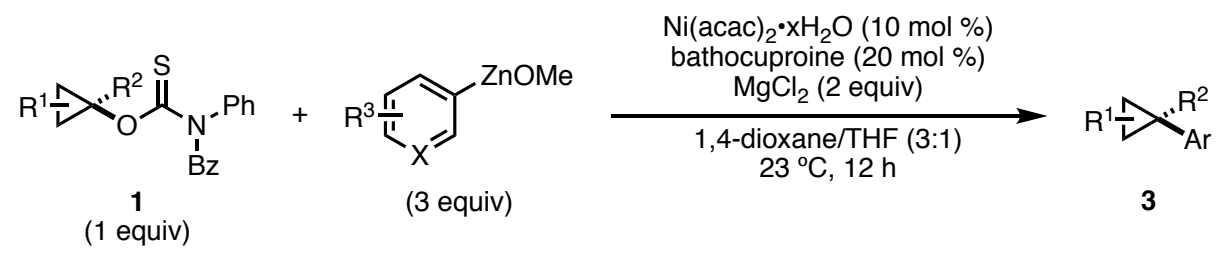

Representative procedure for 0.20 - $\mathrm{mmol}$ scale reaction: A $16-\mathrm{mL}$ threaded culture tube was equipped with a stir bar, sealed with a size 19 septum, and was flame-dried under vacuum and cooled under $\mathrm{N}_{2}$. Thiocarbamate substrate $(0.20 \mathrm{mmol}, 1.0$ equiv), bathocuproine (14 $\mathrm{mg}, 0.040$ mmol, $20 \mathrm{~mol} \%)$, and $\mathrm{Ni}(\mathrm{acac})_{2} \bullet \mathrm{xH}_{2} \mathrm{O}(5.1 \mathrm{mg}, 0.020 \mathrm{mmol}, 10 \mathrm{~mol} \%)$ were added. The tube was sealed, evacuated and backfilled with $\mathrm{N}_{2}(\times 3)$, and brought into a glovebox. Magnesium(II) chloride (38 mg, $0.40 \mathrm{mmol}, 2.0$ equiv) was added. The tube was re-sealed with a septum and electrical tape and was removed from the glovebox. 1,4-Dioxane (anhydrous, degassed; an amount to reach a 3:1 1,4-dioxane/THF ratio, according to the volume of titrated arylzinc(II) methoxide reagent required for $0.60 \mathrm{mmol}$ ) was added, and the solution was stirred at r.t. for 5 min. After 5 min at r.t., the solution turns pink-orange (Figure S1). While stirring, arylzinc solution ( $0.60 \mathrm{mmol}$ of a solution in THF, 3.0 equiv) was added at once, and the reaction was stirred at r.t. $\left(23^{\circ} \mathrm{C}\right)$ for $12 \mathrm{~h}$. The reaction was opened to air and quenched with sat. aq. $\mathrm{NH}_{4} \mathrm{Cl}$. If analyzing by GC-MS, $n$-dodecane was added as internal GC-MS standard. The solution was extracted with EtOAc $(\times 3)$ and the organic fractions were combined, washed with $\mathrm{H}_{2} \mathrm{O}(\times 1)$ and brine ( $\times 1$ ), dried over $\mathrm{MgSO}_{4}$, and concentrated. (The sulfur-containing aqueous waste was treated with bleach and disposed of appropriately.) If analyzing by ${ }^{1} \mathrm{H} N \mathrm{NR}$, dibromomethane was added as internal ${ }^{1} \mathrm{H}$ NMR standard. The crude residue was purified by flash column chromatography to yield the desired cyclopropane.

General Procedure B: Synthesis of cyclopropanes from thiobenzoates

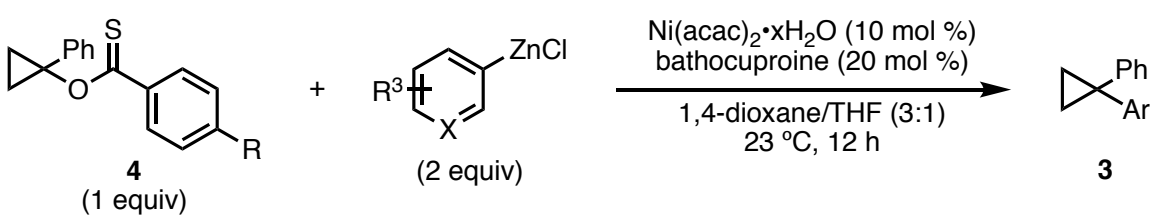

Reactions using thiobenzoates (4) as starting materials were performed as described in General Procedure A with the following modifications: thiobenzoate (4) (1.0 equiv) instead of thiocarbamate substrate, arylzinc(II) chloride (2.0 equiv) instead of arylzinc(II) methoxide, and no $\mathrm{MgCl}_{2}$ was added. 

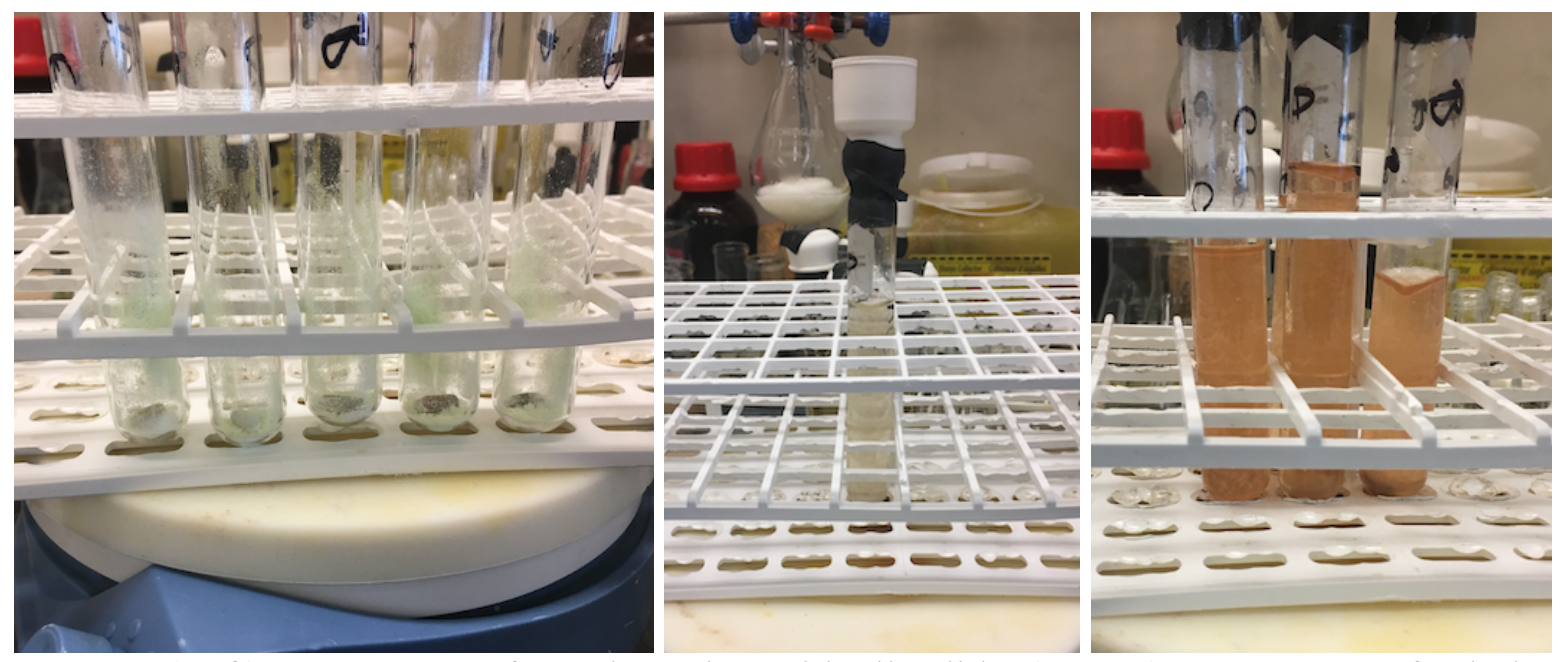

Figure S1. (Left) Appearance of reaction tubes with all solids; (Centre) Appearance of solution immediately after adding 1,4-dioxane; (Right) Appearance after stirring in 1,4-dioxane at r.t. for 5 min.
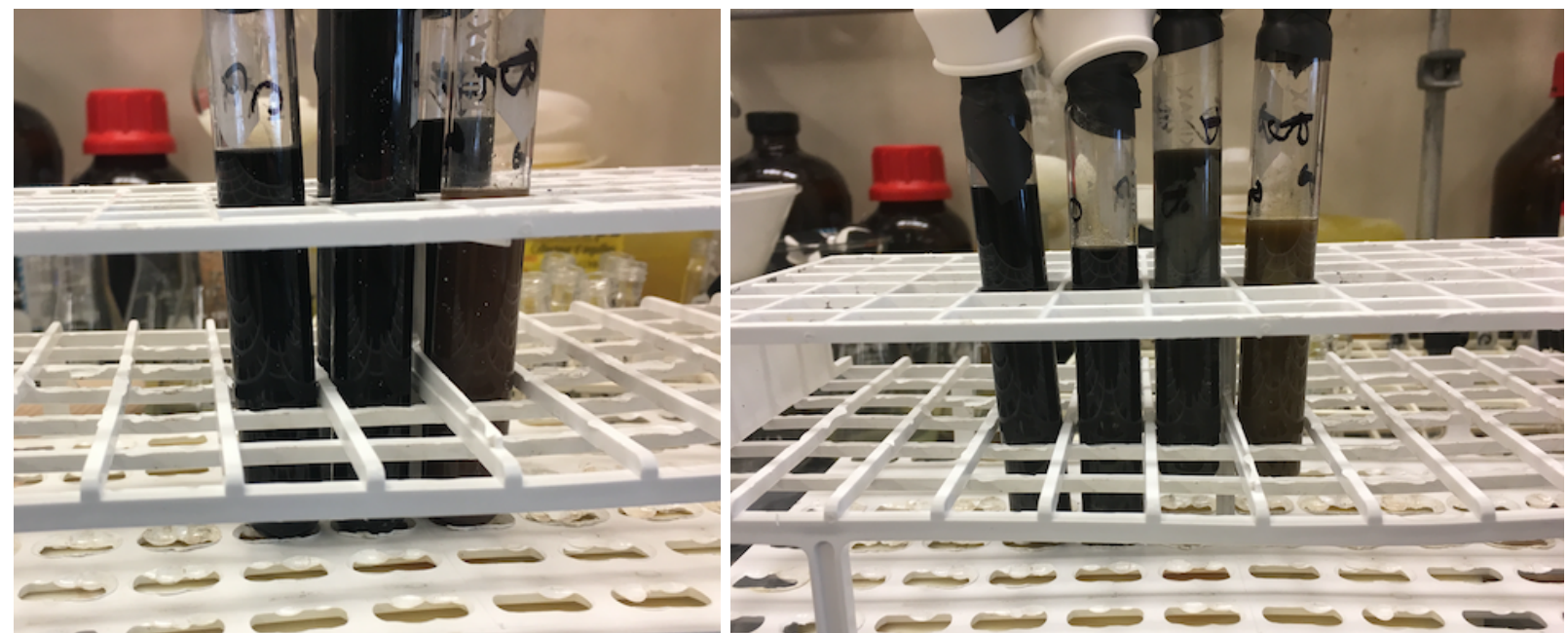

Figure S2. (Left) Appearance of reactions after stirring with arylzinc reagent for ca. $1 \mathrm{~min}$; (Right) Appearance of reactions after stirring for $12 \mathrm{~h}$. 
Table S8. Complete scope table

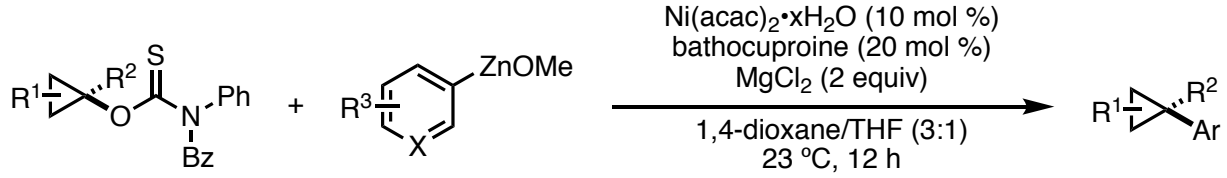

$$
\begin{aligned}
& \begin{array}{c}
1 \\
\text { (1 equiv) }
\end{array}
\end{aligned}
$$

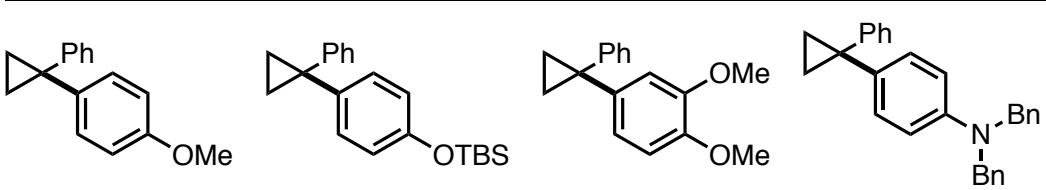

3a, $67 \%$

3n, $60 \%$

3o, $60 \%$

3p, $53 \%$<smiles>Cn1c2ccccc2c2ccc(C3(c4ccccc4)CC3)cc21</smiles>

$3 \mathbf{q}, 32 \%$

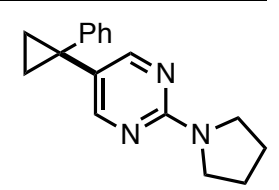

$3 r, 57 \%$<smiles>c1ccc(CN2CCN(c3ncc(C4(c5ccccc5)CC4)cn3)CC2)cc1</smiles><smiles>OC(c1ccccc1)(c1ccccc1)c1ccc(C2(c3ccccc3)CC2)cc1</smiles><smiles>CCCOc1ccc(C2(c3ccccc3)CC2)cc1</smiles><smiles>CCCOc1ccc(C2(c3ccccc3)CC2)cc1</smiles><smiles></smiles>

$3 z, 42 \%$

3y, $48 \%$<smiles>c1ccc(C2(c3ccc4sccc4c3)CC2)cc1</smiles>

$3 u, 36 \%$ *<smiles>CC(C)C1CC[C@@H](C)C[C@H]1Oc1ccc(C2(c3ccccc3)CC2)cc1</smiles>
3ab, $39 \%$<smiles>c1ccc(Oc2ccc(C3(c4ccccc4)CC3)cc2)cc1</smiles>

3ac, 19\%

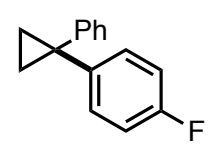

3b, $49 \%$ *

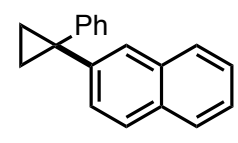

3t, $74 \%{ }^{\star}$

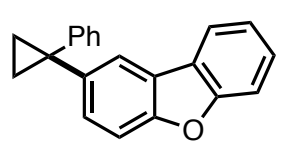

$3 v, 49 \% *$

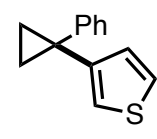

$3 w, 43 \% *$<smiles>c1ccc(C2(c3ccc(N4CCOCC4)cc3)CC2)cc1</smiles>

3ad, $40 \%$<smiles>COc1ccc(C2(c3ccco3)CC2)cc1</smiles>

3c, $66 \%$

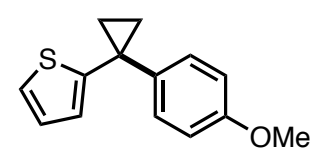

3d, $72 \%$

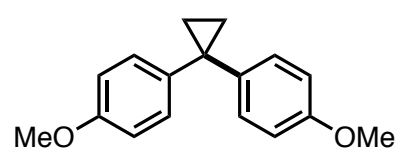

3e, $53 \%$

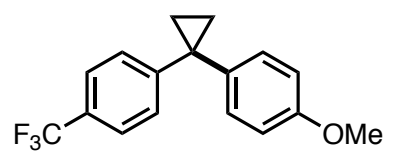

3f, $45 \%$<smiles>COc1ccc(C2(c3ccc(F)cc3)CC2)cc1</smiles>

3ae, $44 \%$<smiles>COc1ccc(C2(c3ccc(Cl)cc3)CC2)cc1</smiles>

3g, $39 \%$<smiles>COc1ccc(C2(/C=C/c3ccccc3)CC2)cc1</smiles>

3h, $96 \%$<smiles>COc1ccc(C2(c3ccc(-c4ccccc4)cc3)CC2)cc1</smiles>

3af, $44 \%$<smiles>COc1ccc(C23CC2COc2ccccc23)cc1</smiles><smiles>COc1ccc(C23CC2(C)CCc2ccccc23)cc1</smiles><smiles>COc1ccc(C23CC2Cc2ccccc23)cc1</smiles>

3I, $43 \%$<smiles>COc1ccc(C2(c3ccc(C(C)(C)C)cc3)CC2)cc1</smiles>

3ag, $45 \%$<smiles>COc1ccc(C2(/C=C/C3=C(C)CCCC3(C)C)CC2)cc1</smiles>

3i, $73 \%$<smiles>c1csc(C2(c3ccc4c(c3)OCO4)CC2)c1</smiles>

3ah, $43 \%$<smiles>c1ccc(C2(c3cccs3)CC2)cc1</smiles>

3ai, $43 \%$<smiles>COc1ccc(C2(Br)CC2)cc1</smiles>

$3 \mathrm{~m}, 67 \%$

1k. See experimental data for details. 


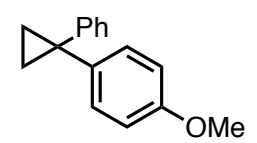

1-Methoxy-4-(1-phenylcyclopropyl)benzene (3a): Prepared on 0.30-mmol scale. The crude residue was purified by flash column chromatography (gradient of $0-40 \% \mathrm{PhMe} /$ hexanes) to yield the product as a colourless oil (44 mg, $0.200 \mathrm{mmol}, 67 \%)$. Analytical data: ${ }^{1} \mathbf{H}$ NMR (400 $\left.\mathrm{MHz}, \mathrm{CDCl}_{3}, 298 \mathrm{~K}\right): \delta_{\mathrm{H}} 7.31-7.13(\mathrm{~m}, 7 \mathrm{H}), 6.88-6.80(\mathrm{~m}, 2 \mathrm{H}), 3.80(\mathrm{~s}, 3 \mathrm{H}), 1.34-1.20(\mathrm{~m}, 4 \mathrm{H})$ ppm; ${ }^{13} \mathbf{C ~ N M R}\left(100 \mathrm{MHz}, \mathrm{CDCl}_{3}, 298 \mathrm{~K}\right): \delta_{\mathrm{C}} 157.9,146.3,137.8,129.9,128.2,127.9,125.7$, 113.7, 55.3, 29.2, 16.3 ppm; HRMS $m / z$ (DART): calcd for $\mathrm{C}_{16} \mathrm{H}_{20} \mathrm{NO}\left(\mathrm{M}+\mathrm{NH}_{4}\right)$ : 242.1539; found: 242.1542; IR (neat): 3081, 3003, 2835, 1604, 1511, 1496, 1458, 1243, 1173, 1028, 825, $756 \mathrm{~cm}^{-1}$; GC-MS m/z (\% relative intensity): 224[M+1 (86), 193 (66), 165 (43), 152 (45), 147 (26), 115 (100), 91 (26); $\mathbf{R}_{\mathbf{f}}(50 \% \mathrm{PhMe} /$ hexanes; UV/p-anisaldehyde): 0.51.

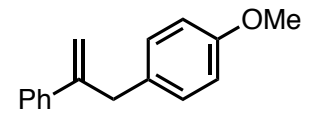

1-Methoxy-4-(2-phenylallyl)benzene (2a): The product was prepared on 0.10-mmol scale. To a 16- $\mathrm{mL}$ threaded culture tube with a stir bar was added $1 \mathbf{a}(91 \mathrm{mg}, 0.30 \mathrm{mmol}, 1.0$ equiv) and $\mathrm{NiCl}_{2}\left(\mathrm{PPh}_{3}\right)_{2}(20 \mathrm{mg}, 0.030 \mathrm{mmol}, 10 \mathrm{~mol} \%)$, and the tube was sealed and evacuated and backfilled with $\mathrm{N}_{2}(\times 3)$. 1,4-Dioxane $(8.7 \mathrm{~mL})$ was added and the reaction was stirred at r.t. for 5 min. 4-Methoxyphenylzinc(II) methoxide $(2.9 \mathrm{~mL}$ of a $0.31 \mathrm{M}$ solution in THF, $0.90 \mathrm{mmol}$, 3.0 equiv) was added and the reaction was stirred at $110^{\circ} \mathrm{C}$ for $16 \mathrm{~h}$. The reaction was cooled to r.t., quenched with sat. aq. $\mathrm{NH}_{4} \mathrm{Cl}$, extracted with EtOAc $(\times 2)$, and the organic fractions were combined, dried over $\mathrm{MgSO}_{4}$, and concentrated. The crude residue was purified by flash column chromatography (gradient of $0-10 \%$ EtOAc/hexanes) to yield the product as a colourless oil (55 $\mathrm{mg}, 0.246 \mathrm{mmol}, 82 \%) .{ }^{1} \mathbf{H}$ NMR $\left(400 \mathrm{MHz}, \mathrm{CDCl}_{3}, 298 \mathrm{~K}\right): \delta_{\mathrm{H}} 7.50-7.43(\mathrm{~m}, 2 \mathrm{H}), 7.36-7.24$ $(\mathrm{m}, 3 \mathrm{H}), 7.21-7.14(\mathrm{~m}, 2 \mathrm{H}), 6.89-6.81(\mathrm{~m}, 2 \mathrm{H}), 5.50(\mathrm{app} \mathrm{d}, J=1.4 \mathrm{~Hz}, 1 \mathrm{H}), 5.04$ (app dd, $J=$ 2.8, $1.4 \mathrm{~Hz}, 1 \mathrm{H}), 3.84-3.78(\mathrm{~m}, 5 \mathrm{H}) \mathrm{ppm} ;{ }^{13} \mathbf{C} \mathbf{N M R}\left(100 \mathrm{MHz}, \mathrm{CDCl}_{3}, 298 \mathrm{~K}\right): \delta_{\mathrm{C}} 158.1,147.5$, 141.0, 131.7, 130.0, 128.4, 127.5, 126.3, 114.4, 113.9, 55.4, 40.9 ppm; GC-MS $\mathrm{m} / z$ (\% relative

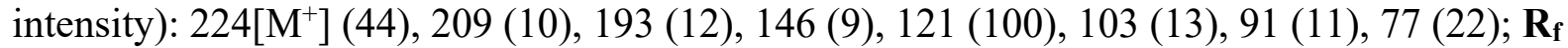
(9:1 hexanes/EtOAc; UV/KMnO 4$): 0.79$.

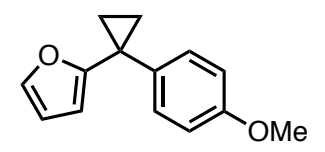

2-(1-(4-Methoxyphenyl)cyclopropyl)furan (3c): Prepared on 0.20-mmol scale. The crude residue was purified by flash column chromatography $(\mathrm{PhMe})$ to yield the product as a colourless oil (28 mg, $0.131 \mathrm{mmol}, 66 \%) .{ }^{1} \mathbf{H}$ NMR $\left(500 \mathrm{MHz}, \mathrm{CDCl}_{3}, 298 \mathrm{~K}\right): \delta_{\mathrm{H}} 7.30-7.26(\mathrm{~m}$, 2H), 7.25-7.23 (m, 1H), 6.88-6.82 (m, 2H), 6.25-6.20 (m, 1H), 5.81-5.76 (m, 1H), 3.80 (s, 3H), 1.39-1.35 (m, 2H), 1.20-1.16 (m, 2H) ppm; ${ }^{13} \mathbf{C}$ NMR (125 MHz, $\left.\mathrm{CDCl}_{3}, 298 \mathrm{~K}\right): \delta_{\mathrm{C}} 159.4$, $158.5,140.8,135.0,130.4,113.8,110.3,105.3,55.4,24.1,14.9$ ppm; HRMS $m / z$ (DART): calcd for $\mathrm{C}_{14} \mathrm{H}_{15} \mathrm{O}_{2}(\mathrm{M}+\mathrm{H}): 215.1067$; found: 215.1067; IR (neat): 3002, 2836, 1611, 1581, 1512, 1462, 1293, 1243, 1159, 1032, 1007, 832, 802, 728, $582 \mathrm{~cm}^{-1} ; \mathbf{R}_{\mathbf{f}}(40 \% \mathrm{PhMe} / \mathrm{hexanes;} \mathrm{UV/p-}$ anisaldehyde): 0.59 . 


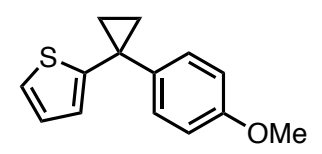

2-(1-(4-Methoxyphenyl)cyclopropyl)thiophene (3d): Prepared on 0.20-mmol scale. The crude residue was purified by flash column chromatography (gradient of $0-40 \% \mathrm{PhMe} / \mathrm{hexanes}$ ) to yield the product as a colourless oil (33 mg, $0.143 \mathrm{mmol}, 72 \%) .{ }^{1} \mathbf{H} \mathbf{~ N M R}\left(500 \mathrm{MHz}, \mathrm{CDCl}_{3}\right.$, $298 \mathrm{~K}): \delta_{\mathrm{H}} 7.35-7.27(\mathrm{~m}, 2 \mathrm{H}), 7.10-7.03(\mathrm{~m}, 1 \mathrm{H}), 6.91-6.82(\mathrm{~m}, 3 \mathrm{H}), 6.71-6.65(\mathrm{~m}, 1 \mathrm{H}), 3.80$ $(\mathrm{s}, 3 \mathrm{H}), 1.38-1.30(\mathrm{~m}, 4 \mathrm{H}) \mathrm{ppm} ;{ }^{13} \mathbf{C}$ NMR $\left(125 \mathrm{MHz}, \mathrm{CDCl}_{3}, 298 \mathrm{~K}\right): \delta_{\mathrm{C}} 158.4,152.6,137.1$, 130.2, 126.7, 123.7, 123.0, 113.8, 55.4, 25.8, 18.4 ppm; HRMS $m / z$ (DART): calcd for $\mathrm{C}_{14} \mathrm{H}_{15} \mathrm{OS}(\mathrm{M}+\mathrm{H}): 231.0838$; found: 231.0842; IR (neat): 3004, 2943, 2834, 1161, 1511, 1441, 1242, 1176, 1032, 827, $691 \mathrm{~cm}^{-1}$; $\mathbf{R}_{\mathbf{f}}$ (40\% PhMe/hexanes; UV/p-anisaldehyde): 0.61.<smiles>COc1ccc(C2(c3ccc(OC)cc3)CC2)cc1</smiles>

4,4'-(Cyclopropane-1,1-diyl)bis(methoxybenzene) (3e): Prepared on 0.074-mmol scale. The crude residue was purified by flash column chromatography (gradient of 20-80\% $\mathrm{PhMe} /$ hexanes) to yield the product as a white solid (10 mg, $0.039 \mathrm{mmol}, 53 \%)$. Analytical data: ${ }^{2} \mathbf{H}$ NMR $\left(500 \mathrm{MHz}, \mathrm{CDCl}_{3}, 298 \mathrm{~K}\right): \delta_{\mathrm{H}} 7.17-7.13(\mathrm{~m}, 4 \mathrm{H}), 6.82-6.78(\mathrm{~m}, 4 \mathrm{H}), 3.78(\mathrm{~s}$, $6 \mathrm{H}), 1.21$ (s, 4H) ppm; ${ }^{13} \mathbf{C}$ NMR (125 MHz, $\left.\mathrm{CDCl}_{3}, 298 \mathrm{~K}\right): \delta_{\mathrm{C}} 157.7,138.3,129.3,113.6,55.3$, 28.5, $16.0 \mathrm{ppm} ; \mathbf{R}_{\mathbf{f}}(40 \% \mathrm{PhMe} /$ hexanes; UV/p-anisaldehyde): 0.42 .<smiles>COc1ccc(C2(c3ccc(C(F)(F)F)cc3)CC2)cc1</smiles>

1-Methoxy-4-(1-(4-(trifluoromethyl)phenyl)cyclopropyl)benzene (3f): Prepared on 0.20mmol scale. The crude residue was purified by flash column chromatography (gradient of $0-40 \%$ $\mathrm{PhMe} /$ hexanes) to yield the product as a colourless oil (26 mg, $0.089 \mathrm{mmol}, 45 \%)$. Analytical data: ${ }^{2} \mathbf{H}$ NMR $\left(400 \mathrm{MHz}, \mathrm{CDCl}_{3}, 298 \mathrm{~K}\right): \delta_{\mathrm{H}} 7.56-7.48(\mathrm{~m}, 2 \mathrm{H}), 7.32-7.20(\mathrm{~m}, 4 \mathrm{H}), 6.92-6.85$ (m, 2H), $3.83(\mathrm{~s}, 3 \mathrm{H}), 1.43-1.25(\mathrm{~m}, 4 \mathrm{H}) \mathrm{ppm} ;{ }^{19} \mathbf{F}$ NMR $\left(376 \mathrm{MHz}, \mathrm{CDCl}_{3}, 298 \mathrm{~K}\right): \delta_{\mathrm{F}}-62.3$ ppm; ${ }^{13} \mathbf{C}$ NMR $\left(125 \mathrm{MHz}, \mathrm{CDCl}_{3}, 298 \mathrm{~K}\right): \delta_{\mathrm{C}} 158.4,150.7(\mathrm{q}, J=1.1 \mathrm{~Hz}), 136.7,130.3,127.9$ $(\mathrm{q}, J=32.2 \mathrm{~Hz}), 127.9,125.3(\mathrm{q}, J=15.2 \mathrm{~Hz}), 124.5(\mathrm{q}, J=270.4 \mathrm{~Hz}), 114.0,55.4,29.3,16.9$ ppm; IR (neat): 3007, 2838, 1615, 1513, 1461, 1410, 1323, 1246, 1164, 1117, 1077, 828, 672 $\mathrm{cm}^{-1} ; \mathbf{R}_{\mathbf{f}}(40 \% \mathrm{PhMe} /$ hexanes; UV/p-anisaldehyde): 0.62 .<smiles>COc1ccc(C2(c3ccc(Cl)cc3)CC2)cc1</smiles>

1-Chloro-4-(1-(4-methoxyphenyl)cyclopropyl)benzene (3g): Prepared on 0.20-mmol scale. The crude residue was purified by flash column chromatography (gradient of $0-30 \%$ $\mathrm{PhMe} /$ hexanes) to yield the product as a colourless oil that solidified upon standing to form a white solid (20 mg, $0.077 \mathrm{mmol}, 39 \%) .{ }^{1} \mathbf{H} \mathbf{N M R}\left(500 \mathrm{MHz}, \mathrm{CDCl}_{3}, 298 \mathrm{~K}\right)$ : $\delta_{\mathrm{H}} 7.23-7.15$ (m, 4H), 7.14-7.09 (m, 2H), 6.85-6.80 (m, 2H), 3.79 (s, 3H), 1.30-1.20 (m, 4H) ppm; ${ }^{13} \mathbf{C ~ N M R}$ (125 MHz, $\left.\mathrm{CDCl}_{3}, 298 \mathrm{~K}\right): \delta_{\mathrm{C}} 158.2,145.0,137.3,131.6,129.9,129.4,128.4,113.9,55.4,28.9$, 16.5 ppm; HRMS m/z (DART): calcd for $\mathrm{C}_{16} \mathrm{H}_{16} \mathrm{OCl}(\mathrm{M}+\mathrm{H})$ : 259.0884; found: 259.0887; IR 
(neat): $3005,2997,2835,1609,1511,1489,1461,1290,1242,1176,1033,1011,818 \mathrm{~cm}^{-1}$; m.p.: $33-36{ }^{\circ} \mathrm{C} ; \mathbf{R}_{\mathbf{f}}(40 \% \mathrm{PhMe} / \mathrm{hexanes;} \mathrm{UV} / p$-anisaldehyde): 0.67.

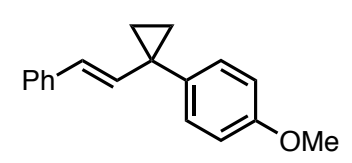

(E)-1-Methoxy-4-(1-styrylcyclopropyl)benzene (3h): Prepared on 0.20-mmol scale. The crude residue was purified by flash column chromatography (gradient of $0-60 \% \mathrm{PhMe} /$ hexanes) to yield the product as a white solid (48 mg, $0.192 \mathrm{mmol}, 96 \%) .{ }^{1} \mathbf{H} \mathbf{~ N M R}\left(500 \mathrm{MHz}, \mathrm{CDCl}_{3}, 298\right.$ $\mathrm{K}): \delta_{\mathrm{H}} 7.31-7.27(\mathrm{~m}, 2 \mathrm{H}), 7.26-7.22(\mathrm{~m}, 4 \mathrm{H}), 7.17-7.12(\mathrm{~m}, 1 \mathrm{H}), 6.91-6.85(\mathrm{~m}, 2 \mathrm{H}), 6.07(\mathrm{~d}, J$ $=15.8 \mathrm{~Hz}, 1 \mathrm{H}), 5.92(\mathrm{~d}, J=15.8 \mathrm{~Hz}, 1 \mathrm{H}), 3.83(\mathrm{~s}, 3 \mathrm{H}), 1.18-1.13(\mathrm{~m}, 2 \mathrm{H}), 1.09-1.04(\mathrm{~m}, 2 \mathrm{H})$ ppm; ${ }^{13} \mathbf{C}$ NMR $\left(125 \mathrm{MHz}, \mathrm{CDCl}_{3}, 298 \mathrm{~K}\right): \delta_{\mathrm{C}} 158.3,138.6,137.7,135.4,131.3,128.6,127.8$, 126.8, 125.9, 113.8, 55.4, 28.0, 15.4 ppm; HRMS $m / z$ (DART): calcd for $\mathrm{C}_{18} \mathrm{H}_{19} \mathrm{O}(\mathrm{M}+\mathrm{H})$ : 251.1430; found: 251.1434; IR (neat): 3085, 3029, 2998, 2953, 1642, 1611, 1511, 1494, 1444, 1291, 1237, 1169, 1029, 966, 932, 834, 747, $693 \mathrm{~cm}^{-1}$; m.p.: $39-41{ }^{\circ} \mathrm{C}$; $\mathbf{R}_{\mathbf{f}}(40 \% \mathrm{PhMe} / \mathrm{hexanes}$; $\mathrm{UV} / p$-anisaldehyde): 0.56 .

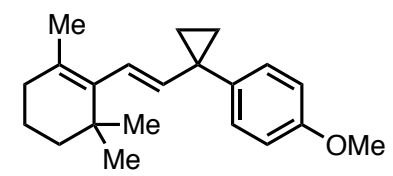

(E)-1-Methoxy-4-(1-(2-(2,6,6-trimethylcyclohex-1-en-1-yl)vinyl)cyclopropyl)benzene (3i): Prepared according to General Procedure A on $0.30-\mathrm{mmol}$ scale. The crude residue was purified by flash column chromatography (gradient of $10-15 \% \mathrm{PhMe} /$ hexanes) to yield the desired product as a clear colourless oil (64 mg, $0.22 \mathrm{mmol}, 73 \%) .{ }^{1} \mathbf{H}$ NMR (400 $\mathrm{MHz}, \mathrm{CDCl}_{3}, 298 \mathrm{~K}$ ): $\delta_{\mathrm{H}} 7.25-7.21(\mathrm{~m}, 2 \mathrm{H}), 6.88-6.78(\mathrm{~m}, 2 \mathrm{H}), 5.47(\mathrm{dtt}, J=15.8,1.9,1.0 \mathrm{~Hz}, 1 \mathrm{H}), 5.31(\mathrm{~d}, J=15.8$ $\mathrm{Hz}, 1 \mathrm{H}), 3.80(\mathrm{~s}, 3 \mathrm{H}), 1.97-1.89(\mathrm{~m}, 2 \mathrm{H}), 1.62(\mathrm{q}, J=1.0 \mathrm{~Hz}, 3 \mathrm{H}), 1.60-1.51(\mathrm{~m}, 3 \mathrm{H}), 1.44-1.36$ $(\mathrm{m}, 2 \mathrm{H}), 1.06-1.00(\mathrm{~m}, 2 \mathrm{H}), 0.97-0.92(\mathrm{~m}, 2 \mathrm{H}), 0.90(\mathrm{~s}, 6 \mathrm{H}) \mathrm{ppm} ;{ }^{13} \mathbf{C} \mathbf{N M R}\left(100 \mathrm{MHz}, \mathrm{CDCl}_{3}\right.$, $298 \mathrm{~K}): \delta_{\mathrm{C}} 157.9,140.6,137.5,136.2,130.4,127.4,125.8,113.5,55.2,39.4,34.1,32.6,28.7$, 27.4, 21.3, 19.3, 14.6 ppm; HRMS $m / z$ (DART): calcd for $\mathrm{C}_{14} \mathrm{H}_{23} \mathrm{O}(\mathrm{M}+\mathrm{H}): 207.1743$; found: 207.1748; IR (neat): 3019, 3000, 2960, 2926, 2863, 2833, 1641, 1612, 1514, 1456, 1241, 1172, 1037, 830, $556 \mathrm{~cm}^{-1} ; \mathbf{R}_{\mathbf{f}}$ (8:2 hexanes/toluene; UV, $p$-anisaldehyde): 0.60 .

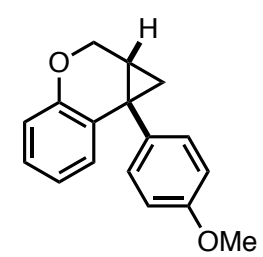

(cis)-7b-(4-Methoxyphenyl)-1,1a,2,7b-tetrahydrocyclopropachromene (3j): Prepared on $0.20-\mathrm{mmol}$ scale. The crude residue was purified by flash column chromatography (gradient of 0-30\% EtOAc/hexanes) to yield the product as a white solid (trial 1: $32 \mathrm{mg}, 0.127 \mathrm{mmol}, 64 \%$; trial 2: $31 \mathrm{mg}, 0.123 \mathrm{mmol}, 62 \%) .{ }^{1} \mathbf{H}$ NMR $\left(500 \mathrm{MHz}, \mathrm{CDCl}_{3}, 298 \mathrm{~K}\right): \delta_{\mathrm{H}} 7.36-7.31(\mathrm{~m}, 2 \mathrm{H})$, 7.04 (ddd, $J=8.0,7.0,2.0 \mathrm{~Hz}, 1 \mathrm{H}), 6.94-6.90(\mathrm{~m}, 2 \mathrm{H}), 6.85(\mathrm{ddd}, J=8.0,1.2,0.4 \mathrm{~Hz}, 1 \mathrm{H}), 6.78$ (ddd, $J=7.7,7.0,1.2 \mathrm{~Hz}, 1 \mathrm{H}), 6.75$ (ddd, $J=7.7,2.0,0.4 \mathrm{~Hz}, 1 \mathrm{H}), 4.42$ (dd, $J=10.6,1.4 \mathrm{~Hz}$, $1 \mathrm{H}), 4.13-4.08(\mathrm{~m}, 1 \mathrm{H}), 3.84(\mathrm{~s}, 3 \mathrm{H}), 1.85$ (ddt, $J=8.6,5.7,1.5 \mathrm{~Hz}, 1 \mathrm{H}), 1.53-1.45(\mathrm{~m}, 2 \mathrm{H})$ ppm; ${ }^{13} \mathrm{C}$ NMR $\left(125 \mathrm{MHz}, \mathrm{CDCl}_{3}, 298 \mathrm{~K}\right): \delta_{\mathrm{C}} 158.7,152.4,135.0,131.8,130.8,128.5,126.3$, 121.4, 117.2, 114.0, 62.8, 55.4, 26.9, 25.5, $16.2 \mathrm{ppm}$; HRMS $m / z$ (DART): calcd for $\mathrm{C}_{17} \mathrm{H}_{17} \mathrm{O}_{2}$ 
$(\mathrm{M}+\mathrm{H})$ : 253.1223; found: 253.1220; IR (neat): 2958, 2838, 2005, 1610, 1578, 1513, 1484, 1451, 1241, 1210, 1036, 968, 820, $765 \mathrm{~cm}^{-1}$; m.p.: $62-64{ }^{\circ} \mathrm{C}$; $\mathbf{R}_{\mathbf{f}}(40 \% \mathrm{PhMe} /$ hexanes; $\mathrm{UV} / p$ anisaldehyde): 0.29 .

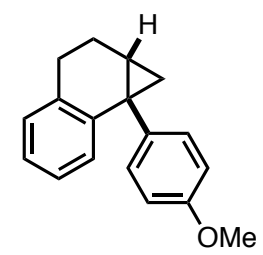

(cis)-7b-(4-Methoxyphenyl)-1a,2,3,7b-tetrahydro-1H-cyclopropanaphthalene (3k): Prepared on $0.20-\mathrm{mmol}$ scale. The crude residue was purified by flash column chromatography (gradient of $0-60 \% \mathrm{PhMe} /$ hexanes) to yield the product as a colourless oil (trial 1: $26 \mathrm{mg}, 0.104 \mathrm{mmol}$, 52\%; trial 2: $28 \mathrm{mg}, 0.112 \mathrm{mmol}, 56 \%) .{ }^{1} \mathbf{H} \mathbf{~ N M R}\left(500 \mathrm{MHz}, \mathrm{CDCl}_{3}, 298 \mathrm{~K}\right): \delta_{\mathrm{H}} 7.33-7.28(\mathrm{~m}$, 2H), 7.09-6.87 (m, 3H), 6.92-6.87 (m, 2H), 6.80-6.76 (m, 1H), 3.84 (s, 3H), 2.79-2.71 (m, 1H), 2.68-2.58 (m, 1H), 2.23-2.14 (m, 1H), 2.06-1.97 (m, 1H), 1.74-1.68 (m, 1H), $1.38(\mathrm{dd}, J=8.6$, $5.0 \mathrm{~Hz}, 1 \mathrm{H}), 1.27-1.24(\mathrm{~m}, 1 \mathrm{H}) \mathrm{ppm} ;{ }^{13} \mathbf{C ~ N M R}\left(125 \mathrm{MHz}, \mathrm{CDCl}_{3}, 298 \mathrm{~K}\right): \delta_{\mathrm{C}} 158.3,142.2$, 137.7, 134.2, 131.8, 128.6, 128.5, 126.0, 124.8, 113.8, 55.4, 27.7, 26.7, 24.1, 19.7, 15.2 ppm; HRMS $m / z$ (DART): calcd for $\mathrm{C}_{18} \mathrm{H}_{19} \mathrm{O}(\mathrm{M}+\mathrm{H}): 251.1430$; found: 251.1430; IR (neat): 3066, 3005, 2925, 2854, 2834, 1746, 1610, 1512, 1489, 1460, 1286, 1242, 1174, 1033, 835, 758, 738 $\mathrm{cm}^{-1} ; \mathbf{R}_{\mathbf{f}}(40 \%$ PhMe/hexanes; UV/KMnO 4$): 0.55$.

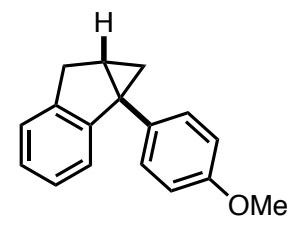

(cis)-1a-(4-Methoxyphenyl)-1,1a,6,6a-tetrahydrocyclopropaindene (3l): Prepared on 0.16mmol scale. The crude residue was purified by flash column chromatography (gradient of $0-40 \%$ $\mathrm{PhMe} /$ hexanes) to yield the product as a white solid (16 mg, $0.068 \mathrm{mmol}, 43 \%)$. ${ }^{\mathbf{1}} \mathbf{H}$ NMR (500 $\left.\mathrm{MHz}, \mathrm{CDCl}_{3}, 298 \mathrm{~K}\right): \delta_{\mathrm{H}} 7.35-7.29(\mathrm{~m}, 2 \mathrm{H}), 7.22-7.16(\mathrm{~m}, 1 \mathrm{H}), 7.13-7.06(\mathrm{~m}, 2 \mathrm{H}), 7.05-7.01$ (m, 1H), 6.90-6.84 (m, 2H), $3.82(\mathrm{~s}, 3 \mathrm{H}), 3.41-3.33(\mathrm{~m}, 1 \mathrm{H}), 3.00$ (dd, $J=17.0 \mathrm{~Hz}, 1 \mathrm{H}), 1.97-$ $1.91(\mathrm{~m}, 1 \mathrm{H}), 1.65(\mathrm{dd}, J=8.3,4.4 \mathrm{~Hz}, 1 \mathrm{H}), 0.52(\mathrm{dd}, J=4.5,4.5 \mathrm{~Hz}, 1 \mathrm{H}) \mathrm{ppm} ;{ }^{13} \mathbf{C} \mathbf{N M R}(125$ $\left.\mathrm{MHz}_{2} \mathrm{CDCl}_{3}, 298 \mathrm{~K}\right): \delta_{\mathrm{C}} 158.4,149.6,141.9,133.6,130.5,126.1,125.7,125.5,123.8,113.8$, 55.4, 39.1, 35.7, 26.0, 21.9 ppm; HRMS $m / z$ (DART): calcd for $\mathrm{C}_{17} \mathrm{H}_{17} \mathrm{O}(\mathrm{M}+\mathrm{H}): 237.1274$; found: 237.1277; IR (neat): 3037, 2995, 2907, 2836, 1611, 1513, 1476, 1459, 1440, 1243, 1176, 1031, 845, 757, $722 \mathrm{~cm}^{-1}$; m.p.: $42-43{ }^{\circ} \mathrm{C}$; $\mathbf{R}_{\mathbf{f}}(40 \%$ PhMe/hexanes; UV/KMnO 4$): 0.63$.

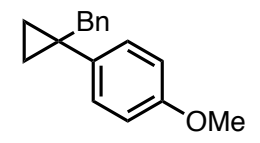

1-(1-Benzylcyclopropyl)-4-methoxybenzene (3m): Prepared on 0.20-mmol scale using General Procedure A with the modifications that 4 equiv 4-methoxyphenylzinc(II) chloride was used (1.8 $\mathrm{mL}$, of a $0.44 \mathrm{M}$ solution in THF, $0.80 \mathrm{mmol}, 4.0$ equiv), $20 \mathrm{~mol} \% \mathrm{Ni}(\mathrm{acac})_{2} \bullet \mathrm{xH}_{2} \mathrm{O}$ was used (10 mg, $0.040 \mathrm{mmol}, 0.20$ equiv), and $40 \mathrm{~mol} \mathrm{\%} \mathrm{bathocuproine} \mathrm{was} \mathrm{used} \mathrm{(} 29 \mathrm{mg}, 0.080 \mathrm{mmol}$, 0.40 equiv). The crude residue was purified by flash column chromatography (gradient of $0-20 \%$ EtOAc/hexanes) to yield the product as a colourless oil (16 mg, $0.066 \mathrm{mmol}, 33 \%) .{ }^{\mathbf{1}} \mathbf{H} \mathbf{~ N M R}$ $\left(500 \mathrm{MHz}, \mathrm{CDCl}_{3}, 298 \mathrm{~K}\right): \delta_{\mathrm{H}} 7.21-7.12(\mathrm{~m}, 3 \mathrm{H}), 7.07-7.03(\mathrm{~m}, 2 \mathrm{H}), 7.00-6.95(\mathrm{~m}, 2 \mathrm{H}), 6.75-$ 
$6.71(\mathrm{~m}, 2 \mathrm{H}), 3.76(\mathrm{~s}, 3 \mathrm{H}), 2.87$ (s, 2H), 0.86-0.75 (m, 4H) ppm; ${ }^{13} \mathbf{C} \mathbf{N M R}\left(125 \mathrm{MHz}, \mathrm{CDCl}_{3}\right.$, $298 \mathrm{~K}): \delta_{\mathrm{C}} 157.8,140.0,137.3,130.4,129.6,128.0,126.0,113.4,55.3,46.2,26.0,12.7 \mathrm{ppm}$; HRMS $m / z$ (DART): calcd for $\mathrm{C}_{17} \mathrm{H}_{22} \mathrm{NO}\left(\mathrm{M}+\mathrm{NH}_{4}\right)$ : 256.1696; found: 256.1697 ; IR (neat): 3029, 3001, 2912, 2836, 1611, 1514, 1454, 1293, 1242, 1174, 1035, 830, 745, $700 \mathrm{~cm}^{-1} ; \mathbf{R}_{\mathbf{f}}(9: 1$ hexanes/EtOAc; UV/p-anisaldehyde): 0.55.

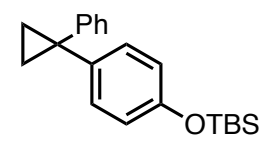

tert-Butyldimethyl(4-(1-phenylcyclopropyl)phenoxy)silane (3n): Prepared on 0.20-mmol scale. The crude residue was purified by flash column chromatography (gradient of 0-20\% $\mathrm{PhMe} /$ hexanes) to yield the product as a colourless oil (39 $\mathrm{mg}, 0.120 \mathrm{mmol}, 60 \%) .{ }^{\mathbf{1}} \mathbf{H} \mathbf{~ N M R}$ $\left(500 \mathrm{MHz}, \mathrm{CDCl}_{3}, 298 \mathrm{~K}\right): \delta_{\mathrm{H}} 7.30-7.22(\mathrm{~m}, 2 \mathrm{H}), 7.22-7.13(\mathrm{~m}, 3 \mathrm{H}), 7.13-7.07(\mathrm{~m}, 2 \mathrm{H}), 6.78-$ $6.71(\mathrm{~m}, 2 \mathrm{H}), 1.30-1.21(\mathrm{~m}, 4 \mathrm{H}), 0.98(\mathrm{~s}, 9 \mathrm{H}), 0.18(\mathrm{~s}, 6 \mathrm{H}) \mathrm{ppm} ;{ }^{13} \mathbf{C} \mathbf{N M R}\left(125 \mathrm{MHz}, \mathrm{CDCl}_{3}\right.$, $298 \mathrm{~K}): \delta_{\mathrm{C}} 153.9,146.4,138.4,129.8,128.3,128.1,125.8,119.8,29.3,25.8,18.3,16.6,-4.3$ ppm; HRMS $m / z$ (DART): calcd for $\mathrm{C}_{21} \mathrm{H}_{29} \mathrm{OSi}(\mathrm{M}+\mathrm{H}): 325.1982$; found: 325.1990 ; IR (neat): 3026, 2956, 2930, 2858, 2006, 1606, 1510, 1472, 1462, 1255, 912, 833, 779, $697 \mathrm{~cm}^{-1} ; \mathbf{R}_{\mathbf{f}}(40 \%$ $\mathrm{PhMe} /$ hexanes; $\mathrm{UV} / p$-anisaldehyde): 0.77.<smiles>COc1ccc(C2(c3ccccc3)CC2)c(OC)c1</smiles>

1,2-Dimethoxy-4-(1-phenylcyclopropyl)benzene (3o): Prepared on 0.10-mmol scale. The crude residue was purified by flash column chromatography on silica gel (gradient of 10-20\% toluene/hexanes) to yield the desired product as a white solid $(15 \mathrm{mg}, 0.060 \mathrm{mmol}, 60 \%) .{ }^{1} \mathbf{H}$ NMR (400 MHz, $\left.\mathrm{CDCl}_{3}, 298 \mathrm{~K}\right): \delta_{\mathrm{H}} 7.26-7.22(\mathrm{~m}, 2 \mathrm{H}), 7.20-7.14(\mathrm{~m}, 3 \mathrm{H}), 6.87-6.77(\mathrm{~m}, 3 \mathrm{H})$, $3.86(\mathrm{~s}, 3 \mathrm{H}), 3.83(\mathrm{~s}, 3 \mathrm{H}), 1.29-1.25(\mathrm{~m}, 4 \mathrm{H}) \mathrm{ppm} ;{ }^{13} \mathbf{C ~ N M R}\left(100 \mathrm{MHz}, \mathrm{CDCl}_{3}, 298 \mathrm{~K}\right): \delta_{\mathrm{C}}$ 148.8, 147.6, 146.3, 138.3, 128.3, 127.7, 125.8, 121.1, 112.6, 111.1, 56.0, 55.9, 29.7, 16.6 ppm; HRMS $m / z$ (DART): calcd for $\mathrm{C}_{17} \mathrm{H}_{19} \mathrm{O}_{2}(\mathrm{M}+\mathrm{H}): 255.1380$; found: 255.1384 ; IR (neat): 3006, 2997, 2969, 2839, 1599, 1498, 1445, 1218, 1138, 1021, 871, 757, 697, $554 \mathrm{~cm}^{-1}$; m.p.: = 43$45^{\circ} \mathrm{C} ; \mathbf{R}_{\mathbf{f}}(7: 3$ hexanes/EtOAc; UV, p-anisaldehyde): 0.67.<smiles>BrCc1ccccc1</smiles>

$\boldsymbol{N}, \boldsymbol{N}$-Dibenzyl-4-(1-phenylcyclopropyl)aniline (3p): Prepared on 0.20-mmol scale. The crude residue was purified by flash column chromatography (gradient of $0-40 \% \mathrm{PhMe} /$ hexanes) to yield the product as a white solid (41 mg, $0.105 \mathrm{mmol}, 53 \%) .{ }^{1} \mathbf{H} \mathbf{~ N M R}\left(500 \mathrm{MHz}, \mathrm{CDCl}_{3}, 298\right.$ $\mathrm{K}): \delta_{\mathrm{H}} 7.35-7.29(\mathrm{~m}, 4 \mathrm{H}), 7.28-7.20(\mathrm{~m}, 10 \mathrm{H}), 7.16-7.11(\mathrm{~m}, 1 \mathrm{H}), 7.06-7.01(\mathrm{~m}, 2 \mathrm{H}), 6.67-6.61$ (m, 2H), 4.62 (s, 4H), 1.21 (app s, 4H) ppm; $\left.{ }^{13} \mathbf{C ~ N M R ~ ( 1 2 5 ~ M H z , ~ C D C l ~} 3,298 \mathrm{~K}\right): \delta_{\mathrm{C}} 147.6$, 146.7, 138.9, 133.9, 129.5, 128.7, 128.4, 128.3, 127.0, 126.8, 125.8, 112.4, 54.5, 29.2, 16.2 ppm; HRMS $m / z$ (DART): calcd for $\mathrm{C}_{29} \mathrm{H}_{28} \mathrm{~N}(\mathrm{M}+\mathrm{H}): 390.2216$; found: 390.2214 ; IR (neat): 3080 , 3023, 2863, 1614, 1602, 1520, 1493, 1386, 1351, 1197, 959, 805, $723 \mathrm{~cm}^{-1}$; m.p.: 88-90 ${ }^{\circ} \mathrm{C}$; $\mathbf{R}_{\mathbf{f}}$ (40\% PhMe/hexanes; UV/p-anisaldehyde/KMnO 4$): 0.53$. 


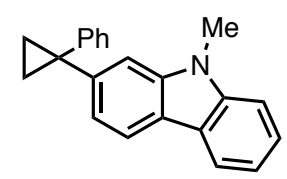

9-Methyl-2-(1-phenylcyclopropyl)-9H-carbazole (3q): Prepared on 0.20-mmol scale. The crude residue was purified by flash column chromatography (gradient of $0-60 \% \mathrm{PhMe} / \mathrm{hexanes}$ ) to yield the product as a white solid (19 mg, $0.064 \mathrm{mmol}, 32 \%) .{ }^{1} \mathbf{H} \mathbf{~ N M R}\left(500 \mathrm{MHz}, \mathrm{CDCl}_{3}\right.$, $298 \mathrm{~K}): \delta_{\mathrm{H}} 8.08-8.04(\mathrm{~m}, 1 \mathrm{H}), 8.03-7.98(\mathrm{~m}, 1 \mathrm{H}), 7.52-7.40(\mathrm{~m}, 1 \mathrm{H}), 7.35-7.31(\mathrm{~m}, 1 \mathrm{H}), 7.30-$ $7.15(\mathrm{~m}, 7 \mathrm{H}), 3.91(\mathrm{~s}, 3 \mathrm{H}), 1.50-1.37(\mathrm{~m}, 4 \mathrm{H}) \mathrm{ppm} ;{ }^{13} \mathbf{C ~ N M R}\left(125 \mathrm{MHz}, \mathrm{CDCl}_{3}, 298 \mathrm{~K}\right): \delta_{\mathrm{C}}$ 146.6, 143.7, 141.5, 141.3, 128.3, 127.9, 125.8, 125.5, 122.8, 121.2, 120.5, 120.3, 120.2, 119.0, 109.1, 108.5, 30.7, 29.2, 17.0 ppm; HRMS $m / z$ (DART): calcd for $\mathrm{C}_{22} \mathrm{H}_{20} \mathrm{~N}(\mathrm{M}+\mathrm{H}): 298.1590$; found: 298.1590; IR (neat): 3050, 1599, 1495, 1466, 1448, 1422, 1322, 1245, 822, 744, 723, 702 $\mathrm{cm}^{-1}$; m.p.: $111-113{ }^{\circ} \mathrm{C} ; \mathbf{R}_{\mathbf{f}}(40 \%$ PhMe/hexanes; UV/p-anisaldehyde): 0.43 .<smiles>c1ccc(C2(c3cnc(N4CCCC4)nc3)CC2)cc1</smiles>

5-(1-Phenylcyclopropyl)-2-(pyrrolidin-1-yl)pyrimidine (3r): Prepared on 0.20-mmol scale. The crude residue was purified by flash column chromatography (gradient of $0-100 \%$ EtOAc/hexanes) to yield the product as a tan solid (30 mg, $0.113 \mathrm{mmol}, 57 \%$ ), which was inseparable from the homodimer side-product. ${ }^{1} \mathbf{H}$ NMR $\left(500 \mathrm{MHz}, \mathrm{CDCl}_{3}, 298 \mathrm{~K}\right)$ : $\delta_{\mathrm{H}} 8.28$ (s, 2H), 7.27-7.22 (m, 2H), 7.19-7.13 (m, 3H), 3.58-3.52 (m, 4H), 2.01-1.94 (m, 4H), 1.29-1.25 (m, 2H), 1.20-1.17 (m, 2H) ppm; ${ }^{13} \mathbf{C}$ NMR (125 MHz, $\left.\mathrm{CDCl}_{3}, 298 \mathrm{~K}\right): \delta_{\mathrm{C}} 160.9,158.9,145.4$, $129.5,127.5,126.1,125.5,46.8,25.7,25.1,15.4 \mathrm{ppm}$; HRMS $m / z$ (DART): calcd for $\mathrm{C}_{17} \mathrm{H}_{20} \mathrm{~N}_{3}$ $(\mathrm{M}+\mathrm{H}): 266.1652$; found: 266.1651; IR (neat): 2969, 2868, 1594, 1524, 1514, 1480, 1281, 1018, 797, $700 \mathrm{~cm}^{-1}$; m.p.: 83-85 ${ }^{\circ} \mathrm{C}$; $\mathbf{R}_{\mathbf{f}}(7: 3$ hexanes/EtOAc; UV/KMnO 4$): 0.42$.

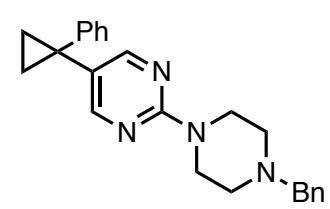

2-(4-Benzylpiperazin-1-yl)-5-(1-phenylcyclopropyl)pyrimidine (3s): Prepared on 0.20-mmol scale. The crude residue was purified by flash column chromatography (gradient of $0-100 \%$ EtOAc/hexanes) to yield the product as a white solid (35 mg, $0.094 \mathrm{mmol}, 47 \%$ ), which was inseparable from the homodimer side-product. ${ }^{1} \mathbf{H}$ NMR $\left(500 \mathrm{MHz}, \mathrm{CDCl}_{3}, 298 \mathrm{~K}\right)$ : $\delta_{\mathrm{H}} 8.25$ (s, 2H), 7.37-7.31 (m, 4H), 7.29-7.23 (m, 3H), 7.19-7.14 (m, 3H), 3.88-3.74 (br m, 4H), 3.56 (br s, 2H), 2.58-2.44 (br m, 4H), 1.31-1.25 (m, 2H), 1.20-1.16 (m, 2H) ppm; ${ }^{13}$ C NMR (125 MHz, $\left.\mathrm{CDCl}_{3}, 298 \mathrm{~K}\right): \delta_{\mathrm{C}} 160.6,158.7,145.1,137.8,129.4,128.6,128.5,127.7,127.4,126.6,126.2$, 63.3, 53.1, 43.9, 25.1, 15.3 ppm; HRMS $m / z$ (DART): calcd for $\mathrm{C}_{24} \mathrm{H}_{27} \mathrm{~N}_{4}(\mathrm{M}+\mathrm{H})$ : 371.2230; found: 371.2236; IR (neat): 3072, 3026, 3000, 2835, 2824, 1598, 1530, 1486, 1451, 1444, 1355 , 1253, 1239, 1006, 960, 931, 797, $734 \mathrm{~cm}^{-1}$; m.p.: $94-96^{\circ} \mathrm{C} ; \mathbf{R}_{\mathbf{f}}(7: 3$ hexanes/EtOAc;

$\left.\mathrm{UV} / \mathrm{KMnO}_{4}\right): 0.28$.

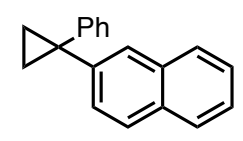


2-(1-Phenylcyclopropyl)naphthalene (3t): Prepared on $0.10-\mathrm{mmol}$ scale according to General Procedure B using 6a $(0.10 \mathrm{mmol})$. The crude residue was purified by flash column chromatography (gradient of $0-30 \% \mathrm{PhMe} /$ hexanes) to yield the product as a colourless oil that slowly solidified upon standing (18 mg, $0.074 \mathrm{mmol}, 74 \%) .{ }^{1} \mathbf{H} \mathbf{N M R}\left(500 \mathrm{MHz}, \mathrm{CDCl}_{3}, 298\right.$ $\mathrm{K}): \delta_{\mathrm{H}} 7.81-7.73(\mathrm{~m}, 3 \mathrm{H}), 7.70-7.63(\mathrm{~m}, 1 \mathrm{H}), 7.47-7.37(\mathrm{~m}, 3 \mathrm{H}), 7.30-7.24(\mathrm{~m}, 4 \mathrm{H}), 7.21-7.16$ $(\mathrm{m}, 1 \mathrm{H}), 1.45-1.35(\mathrm{~m}, 4 \mathrm{H}) \mathrm{ppm} ;{ }^{13} \mathbf{C} \mathbf{N M R}\left(125 \mathrm{MHz}, \mathrm{CDCl}_{3}, 298 \mathrm{~K}\right): \delta_{\mathrm{C}} 145.8,143.2,133.5$, 132.2, 128.4, 128.4, 128.0, 127.8, 127.7, 127.5, 127.0, 126.1, 126.1, 125.6, 30.2, 16.5 ppm; HRMS $m / z$ (DART): calcd for $\mathrm{C}_{19} \mathrm{H}_{17}(\mathrm{M}+\mathrm{H}): 245.1325$; found: 245.1321 ; IR (neat): 3077 , 3055, 3004, 1598, 1497, 1425, 1267, 1197, 1136, 1025, 863, 822, 756, 741, $696 \mathrm{~cm}^{-1}$; m.p.: 40$42{ }^{\circ} \mathrm{C} ; \mathbf{R}_{\mathbf{f}}(40 \%$ PhMe/hexanes; UV/p-anisaldehyde): 0.66.

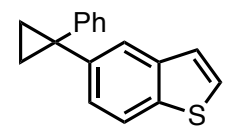

5-(1-Phenylcyclopropyl)benzothiophene (3u): Prepared on 0.20-mmol scale according to General Procedure B using 6a $(0.20 \mathrm{mmol})$. The crude residue was purified by flash column chromatography (gradient of $0-20 \% \mathrm{Et}_{2} \mathrm{O} /$ hexanes) to yield the product as a colourless semisolid (18 mg, $0.072 \mathrm{mmol}, 36 \%) .{ }^{1} \mathbf{H}$ NMR $\left(500 \mathrm{MHz}, \mathrm{CDCl}_{3}, 298 \mathrm{~K}\right): \delta_{\mathrm{H}} 7.78$ (app d, $J=8.5 \mathrm{~Hz}$, 1H), $7.72(\operatorname{app~d}, J=1.9 \mathrm{~Hz}, 1 \mathrm{H}), 7.41(\mathrm{~d}, J=5.4 \mathrm{~Hz}, 1 \mathrm{H}), 7.32-7.20(\mathrm{~m}, 6 \mathrm{H}), 7.19-7.15$ (m, 1H), 1.39-1.32 (m, 4H) ppm; ${ }^{13} \mathbf{C} \mathbf{N M R}\left(125 \mathrm{MHz}, \mathrm{CDCl}_{3}, 298 \mathrm{~K}\right): \delta_{\mathrm{C}} 146.2,142.1,139.9$, $137.7,128.4,128.2,126.8,126.0,125.9,123.9,123.7,122.4,30.1,16.6$ ppm; HRMS $\mathrm{m} / \mathrm{z}$ (DART): calcd for $\mathrm{C}_{17} \mathrm{H}_{15} \mathrm{~S}(\mathrm{M}+\mathrm{H})$ : 251.0889; found: 251.0891; IR (neat): 3077, 2924, 1599, 1494, 1435, 1189, 1044, 1023, 900, 754, $695 \mathrm{~cm}^{-1}$; $\mathbf{R}_{\mathbf{f}}(40 \%$ PhMe/hexanes; UV/KMnO 4$): 0.63$.

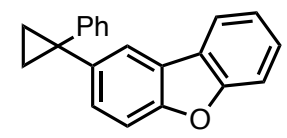

2-(1-Phenylcyclopropyl)dibenzofuran (3v): Prepared on 0.20 -mmol scale according to General Procedure B using 6a $(0.20 \mathrm{mmol})$. The crude residue was purified by flash column chromatography (gradient of $0-20 \% \mathrm{Et}_{2} \mathrm{O} /$ hexanes) to yield the product as a white solid (28 $\mathrm{mg}$, $0.098 \mathrm{mmol}, 49 \%)$. ${ }^{1} \mathbf{H}$ NMR $\left(500 \mathrm{MHz}, \mathrm{CDCl}_{3}, 298 \mathrm{~K}\right): \delta_{\mathrm{H}} 7.93-7.88(\mathrm{~m}, 2 \mathrm{H}), 7.55$ (app dt, $J=$ $8.3,0.8 \mathrm{~Hz}, 1 \mathrm{H}), 7.47$ (dd, $J=8.6,0.7 \mathrm{~Hz}, 1 \mathrm{H}), 7.46-7.40(\mathrm{~m}, 2 \mathrm{H}), 7.32$ (app td, $J=7.5,1.0 \mathrm{~Hz}$, 1H), 7.29-7.20 (m, 4H), 7.19-7.14 (m, 1H), 1.43-1.35 (m, 4H) ppm; ${ }^{13}$ C NMR (125 MHz, $\left.\mathrm{CDCl}_{3}, 298 \mathrm{~K}\right): \delta_{\mathrm{C}} 156.6,154.8,146.3,140.2,128.6,128.3,127.7,127.0,125.8,124.2,124.2$, 122.6, 121.0, 120.6, 111.6, 111.3, 30.0, $16.6 \mathrm{ppm}$; HRMS $\mathrm{m} / z$ (DART): calcd for $\mathrm{C}_{21} \mathrm{H}_{17} \mathrm{O}$ $(\mathrm{M}+\mathrm{H}):$ 285.1274; found: 285.1275; IR (neat): 3055, 2925, 1601, 1495, 1479, 1448, 1344, 1194 , 1116, 1022, 823, 810, 746, $697 \mathrm{~cm}^{-1}$; m.p.: 50-53 ${ }^{\circ} \mathrm{C}$; $\mathbf{R}_{\mathbf{f}}(40 \%$ PhMe/hexanes; UV; $p$ anisaldehyde): 0.68 .<smiles></smiles>

3-(1-Phenylcyclopropyl)thiophene (3w): Prepared on 0.20-mmol scale according to General Procedure B using $\mathbf{6 a}(0.20 \mathrm{mmol})$. The crude residue was purified by flash column chromatography (gradient of $0-20 \% \mathrm{Et}_{2} \mathrm{O} /$ hexanes) to yield the product as a colourless oil (17 mg, $0.085 \mathrm{mmol}, 43 \%) .{ }^{1} \mathbf{H}$ NMR $\left(400 \mathrm{MHz}, \mathrm{CDCl}_{3}, 298 \mathrm{~K}\right): \delta_{\mathrm{H}} 7.33-7.27(\mathrm{~m}, 4 \mathrm{H}), 7.24-7.19$ $(\mathrm{m}, 2 \mathrm{H}), 6.83(\mathrm{dd}, J=5.0,1.4 \mathrm{~Hz}, 1 \mathrm{H}), 6.76(\mathrm{dd}, J=3.0,1.4 \mathrm{~Hz}, 1 \mathrm{H}), 1.31-1.24(\mathrm{~m}, 4 \mathrm{H}) \mathrm{ppm}$; 
${ }^{13}$ C NMR (125 MHz, $\left.\mathrm{CDCl}_{3}, 298 \mathrm{~K}\right): \delta_{\mathrm{C}} 147.8,145.2,129.0,128.4,127.1,126.4,125.5,120.5$, 26.5, 16.8 ppm; HRMS m/z (DART): calcd for $\mathrm{C}_{13} \mathrm{H}_{13} \mathrm{~S}(\mathrm{M}+\mathrm{H}): 201.0733$; found: 201.0733; IR (neat): 3082, 3004, 1601, 1495, 1445, 1203, 1023, 949, 849, 775, 758, $698 \mathrm{~cm}^{-1} ; \mathbf{R}_{\mathbf{f}}(40 \%$ $\mathrm{PhMe} / \mathrm{hexanes;} \mathrm{UV} / p$-anisaldehyde): 0.67.<smiles>Oc1ccc(C2(P)CC2)cc1</smiles>

1-(Benzyloxy)-4-(1-phenylcyclopropyl)benzene (3x): Prepared on 0.30-mmol scale. The crude residue was purified by flash column chromatography on silica gel (gradient of 10-20\% $\mathrm{PhMe} /$ hexanes) to yield the product as a pale yellow solid (44 mg, $0.147 \mathrm{mmol}, 49 \%)$. ${ }^{1} \mathbf{H}$ NMR $\left(400 \mathrm{MHz}, \mathrm{CDCl}_{3}, 298 \mathrm{~K}\right): \delta_{\mathrm{H}} 7.45-7.29(\mathrm{~m}, 5 \mathrm{H}), 7.29-7.23(\mathrm{~m}, 2 \mathrm{H}), 7.23-7.12(\mathrm{~m}, 5 \mathrm{H}), 6.94$ $6.86(\mathrm{~m}, 2 \mathrm{H}), 5.04(\mathrm{~s}, 2 \mathrm{H}), 1.26(\mathrm{~m}, 4 \mathrm{H}) \mathrm{ppm} ;{ }^{13} \mathbf{C} \mathbf{N M R}\left(100 \mathrm{MHz}, \mathrm{CDCl}_{3}, 298 \mathrm{~K}\right): \delta_{\mathrm{C}} 157.2$, 146.3, 138.2, 137.3, 129.9, 128.7, 128.3, 128.1, 128.0, 127.6, 125.8, 114.7, 70.2, 29.3, 16.4 ppm; HRMS $m / z$ (DART): calcd for $\mathrm{C}_{22} \mathrm{H}_{24} \mathrm{NO}\left(\mathrm{M}+\mathrm{NH}_{4}\right)$ : 318.1852 ; found: 318.1851 ; IR (neat): 3075, 3024, 2920, 1605, 1510, 1496, 1455, 1387, 1237, 1174, 1012, 1919, 819, 756, $747 \mathrm{~cm}^{-1}$; m.p.: 59-61 ${ }^{\circ} \mathrm{C} ; \mathbf{R}_{\mathbf{f}}(7: 3$ hexanes/toluene; UV, p-anisaldehyde): 0.48.

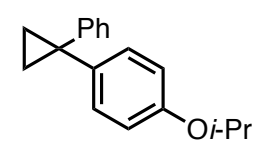

1-Isopropoxy-4-(1-phenylcyclopropyl)benzene (3y): Prepared on 0.40-mmol scale. The crude residue was purified by flash column chromatography (gradient of $0-50 \% \mathrm{PhMe} /$ hexanes) to yield the product as a colourless oil (48 $\mathrm{mg}, 0.190 \mathrm{mmol}, 48 \%) .{ }^{1} \mathbf{H} \mathbf{~ N M R}\left(400 \mathrm{MHz}, \mathrm{CDCl}_{3}\right.$, $298 \mathrm{~K}): \delta_{\mathrm{H}} 7.42-7.14(\mathrm{~m}, 7 \mathrm{H}), 6.92-6.80(\mathrm{~m}, 2 \mathrm{H}), 4.60-4.48(\mathrm{~m}, 1 \mathrm{H}), 1.48-1.25(\mathrm{~m}, 10 \mathrm{H}) \mathrm{ppm}$; ${ }^{13} \mathrm{C}$ NMR (100 MHz, $\left.\mathrm{CDCl}_{3}, 298 \mathrm{~K}\right): \delta_{\mathrm{C}} 156.3,146,4,137.7,129.9,128.3,128.1,125.8,115.7$, 70.0, 29.3, 22.3, $16.5 \mathrm{ppm}$; HRMS $\mathrm{m} / z$ (DART): calcd for $\mathrm{C}_{18} \mathrm{H}_{21} \mathrm{O}(\mathrm{M}+\mathrm{H}): 253.1587$; found: 253.1595; IR (neat): 3082, 2977, 1606, 1509, 1497, 1371, 1238, 1115, 953, 756, $697 \mathrm{~cm}^{-1}$; $\mathbf{R}_{\mathbf{f}}$ (20\% PhMe/hexanes; UV/KMnO 4$): 0.50$.

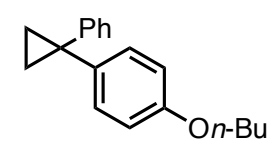

1-Butoxy-4-(1-phenylcyclopropyl)benzene (3z): Prepared on 0.30-mmol scale. The crude residue was purified by flash column chromatography (gradient of $10-15 \% \mathrm{PhMe} / \mathrm{hexanes}$ ) to yield the product as a pale yellow solid (33 mg, $0.13 \mathrm{mmol}, 42 \%) .{ }^{1} \mathbf{H} \mathbf{~ N M R}\left(400 \mathrm{MHz}, \mathrm{CDCl}_{3}\right.$, $298 \mathrm{~K}): \delta_{\mathrm{H}} 7.31-7.22(\mathrm{~m}, 2 \mathrm{H}), 7.22-7.12(\mathrm{~m}, 5 \mathrm{H}), 6.85-6.75(\mathrm{~m}, 2 \mathrm{H}), 3.94(\mathrm{t}, \mathrm{J}=6.5 \mathrm{~Hz}, 2 \mathrm{H})$, $1.80-1.71(\mathrm{~m}, 2 \mathrm{H}), 1.56-1.43(\mathrm{~m}, 3 \mathrm{H}), 0.97(\mathrm{t}, \mathrm{J}=7.4 \mathrm{~Hz}, 3 \mathrm{H}) \mathrm{ppm} ;{ }^{13} \mathbf{C} \mathbf{N M R}(100 \mathrm{MHz}$, $\left.\mathrm{CDCl}_{3}, 298 \mathrm{~K}\right): \delta_{\mathrm{C}} 157.6,146.4,137.6,129.9,128.3,128.0,125.8,114.3,67.8,31.5,29.8,29.3$, 19.4, 16.4, 13.9 ppm; HRMS $m / z$ (DART): calcd for $\mathrm{C}_{19} \mathrm{H}_{23} \mathrm{O}(\mathrm{M}+\mathrm{H}): 267.1743$; found: 267.1744; IR (neat): 3081, 2958, 2933, 2914, 2872, 2861, 1610, 1516, 1497, 1454, 1288, 1241, 1177, 1070, 1021, 969, 921, 838, 816, 754, $696 \mathrm{~cm}^{-1}$; m.p.: $30-32{ }^{\circ} \mathrm{C} ; \mathbf{R}_{\mathbf{f}}(7: 3$ hexanes/toluene; UV, p-anisaldehyde): 0.62. 


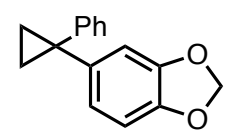

5-(1-Phenylcyclopropyl)benzo[1,3]dioxole (3aa): Prepared on 0.30-mmol scale. The crude residue was purified by flash column chromatography (gradient of $0-40 \% \mathrm{PhMe} / \mathrm{hexanes)}$ to yield the product as a colourless oil (22 $\mathrm{mg}, 0.092 \mathrm{mmol}, 31 \%) .{ }^{1} \mathbf{H}$ NMR $\left(500 \mathrm{MHz}, \mathrm{CDCl}_{3}\right.$, $298 \mathrm{~K}): \delta_{\mathrm{H}} 7.29-7.24(\mathrm{~m}, 2 \mathrm{H}), 7.23-7.14(\mathrm{~m}, 3 \mathrm{H}), 6.81-6.85(\mathrm{~m}, 2 \mathrm{H}), 7.74-7.70(\mathrm{~m}, 1 \mathrm{H}), 5.92$ (s, 2H), 1.32-1.21 (m, 4H) ppm; ${ }^{13}$ C NMR (125 MHz, $\left.\mathrm{CDCl}_{3}, 298 \mathrm{~K}\right): \delta_{\mathrm{C}} 147.6,146.2,145.9$, 139.8, 128.4, 128.0, 126.0, 122.0, 109.7, 108.1, 101.0, 30.0, 16.5 ppm; HRMS $m / z$ (DART): calcd for $\mathrm{C}_{16} \mathrm{H}_{15} \mathrm{O}_{2}(\mathrm{M}+\mathrm{H})$ : 239.1067; found: 239.1073; IR (neat): 3081, 3006, 2890, 1742, $1602,1503,1484,1433,1251,1225,1033,934,808,756,697,560 \mathrm{~cm}^{-1} ; \mathbf{R}_{\mathbf{f}}(40 \%$ $\mathrm{PhMe} /$ hexanes; $\mathrm{UV} / p$-anisaldehyde/ $\left.\mathrm{KMnO}_{4}\right)$ : 0.45 .<smiles>C[C@@H](O)C1CC[C@@H](Oc2ccc(C3CC3)cc2)C[C@H]1O</smiles>

1-(((1R,2S,5R)-2-Isopropyl-5-methylcyclohexyl)oxy)-4-(1-phenylcyclopropyl)benzene (3ab): Prepared on $0.20-\mathrm{mmol}$ scale. The crude residue was purified by flash column chromatography (gradient of $0-20 \% \mathrm{PhMe} /$ hexanes) to yield the product as a colourless oil $(27 \mathrm{mg}, 0.077 \mathrm{mmol}$, $39 \%) .{ }^{1} \mathbf{H}$ NMR $\left(500 \mathrm{MHz}, \mathrm{CDCl}_{3}, 298 \mathrm{~K}\right): \delta_{\mathrm{H}} 7.29-7.11(\mathrm{~m}, 7 \mathrm{H}), 6.83-6.77(\mathrm{~m}, 2 \mathrm{H}), 3.98(\mathrm{td}, J$ $=10.6,4.2 \mathrm{~Hz}, 1 \mathrm{H}), 2.26-2.09(\mathrm{~m}, 2 \mathrm{H}), 1.77-1.66(\mathrm{~m}, 2 \mathrm{H}), 1.58-1.37(\mathrm{~m}, 2 \mathrm{H}), 1.33-1.19(\mathrm{~m}$, 4H), $1.14-1.02(\mathrm{~m}, 1 \mathrm{H}), 1.02-0.85(\mathrm{~m}, 8 \mathrm{H}), 0.77(\mathrm{~d}, J=6.9 \mathrm{~Hz}, 3 \mathrm{H}) \mathrm{ppm} ;{ }^{13} \mathbf{C} \mathbf{N M R}(125 \mathrm{MHz}$, $\left.\mathrm{CDCl}_{3}, 298 \mathrm{~K}\right): \delta_{\mathrm{C}} 156.7,146.5,137.6,129.8,128.3,128.2,125.9,115.7,77.5,48.2,40.4,34.7$, 31.6, 26.1 23.8, 22.3, 20.9, 16.7, $16.4 \mathrm{ppm}$; HRMS $\mathrm{m} / z$ (DART): calcd for $\mathrm{C}_{25} \mathrm{H}_{33} \mathrm{O}(\mathrm{M}+\mathrm{H}$ ): 349.2526; found: 349.2524; IR (neat): 2954, 2924, 2869, 1600, 1508, 1456, 1270, 1241, 1166, 1013, 993, 823, 755, $697 \mathrm{~cm}^{-1} ; \mathbf{R}_{\mathbf{f}}$ (40\% PhMe/hexanes; UV/p-anisaldehyde): 0.68.<smiles>c1ccc(C2(c3ccccc3)CC2)cc1</smiles>

1-Phenoxy-4-(1-phenylcyclopropyl)benzene (3ac): Prepared on 0.40-mmol scale. The crude residue was purified by flash column chromatography (gradient of $0-30 \% \mathrm{PhMe} / \mathrm{hexanes)}$ to yield the product as a white solid (22 $\mathrm{mg}, 0.077 \mathrm{mmol}, 19 \%) .{ }^{1} \mathbf{H}$ NMR (400 MHz, $\mathrm{CDCl}_{3}, 298$ $\mathrm{K}): \delta_{\mathrm{H}} 7.39-7.19(\mathrm{~m}, 9 \mathrm{H}), 7.15-7.09(\mathrm{~m}, 1 \mathrm{H}), 7.07-7.01(\mathrm{~m}, 2 \mathrm{H}), 6.98-6.93(\mathrm{~m}, 2 \mathrm{H}), 1.36-1.28$ (m, 4H) ppm; ${ }^{13}$ C NMR (100 MHz, $\left.\mathrm{CDCl}_{3}, 298 \mathrm{~K}\right): \delta_{\mathrm{C}} 157.5,155.5,146.0,140.8,130.0,129.8$, 128.4, 128.4, 126.1, 123.2, 118.9, 118.8, 29.5, $16.5 \mathrm{ppm}$; HRMS $\mathrm{m} / \mathrm{z}$ (DART): calcd for $\mathrm{C}_{21} \mathrm{H}_{19} \mathrm{O}(\mathrm{M}+\mathrm{H}): 287.1430$; found: 287.1434; IR (neat): 3077, 3003, 1581, 1492, 1477, 1291 , $1162,1015,857,769,752,704 \mathrm{~cm}^{-1}$; m.p.: $83-85^{\circ} \mathrm{C} ; \mathbf{R}_{\mathbf{f}}(20 \%$ PhMe/hexanes; UV/panisaldehyde): 0.56 .

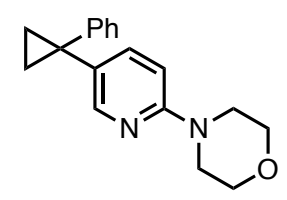

4-(5-(1-Phenylcyclopropyl)pyridin-2-yl)morpholine (3ad): Prepared according to General Procedure A on 0.20 -mmol scale. The crude residue was purified by flash column 
chromatography (gradient of 5-15\% toluene/hexanes) to yield the desired product as a pale yellow solid (22 mg, $0.079 \mathrm{mmol}, 40 \%) .{ }^{1} \mathbf{H}$ NMR (500 MHz, $\left.\mathrm{CDCl}_{3}, 298 \mathrm{~K}\right): \delta_{\mathrm{H}} 8.15$ (dd, $J=$ 2.5, $0.8 \mathrm{~Hz}, 1 \mathrm{H}), 7.43(\mathrm{dd}, J=8.7,2.5 \mathrm{~Hz}, 1 \mathrm{H}), 7.27-7.22(\mathrm{~m}, 2 \mathrm{H}), 7.20-7.13(\mathrm{~m}, 3 \mathrm{H}), 6.58(\mathrm{dd}$, $J=8.8,0.8 \mathrm{~Hz}, 1 \mathrm{H}), 3.87-3.76(\mathrm{~m}, 4 \mathrm{H}), 3.54-3.41(\mathrm{~m}, 4 \mathrm{H}), 1.29-1.25(\mathrm{~m}, 2 \mathrm{H}), 1.24-1.21$ (m, 2H) ppm; ${ }^{13} \mathbf{C}$ NMR $\left(126 \mathrm{MHz}, \mathrm{CDCl}_{3}, 298 \mathrm{~K}\right): \delta_{\mathrm{C}} 158.1,148.2,145.6,138.6,130.8,128.3$, 127.7, 125.9, 106.6, 66.8, 45.8, 27.0, 15.7 ppm; HRMS $m / z$ (DART): calcd for $\mathrm{C}_{18} \mathrm{H}_{21} \mathrm{~N}_{2} \mathrm{O}$ $(\mathrm{M}+\mathrm{H})$ : 281.1648; found: 281.1644; IR (neat): 3021, 2966, 2886, 2844, 1600, 1556, 1494, 1445 , 1391, 1239, 1116, 943, 808, 757, 559, $\mathrm{cm}^{-1}$; m.p.: 59-61 ${ }^{\circ} \mathrm{C} ; \mathbf{R}_{\mathbf{f}}(7: 3$ hexanes/EtOAc; UV, $\left.\mathrm{KMnO}_{4}\right): 0.40$.

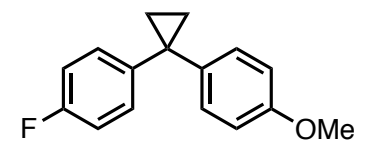

1-Fluoro-4-(1-(4-methoxyphenyl)cyclopropyl)benzene (3ae): Prepared on 0.20-mmol scale. The crude residue was purified by flash column chromatography (gradient of $0-30 \%$ $\mathrm{PhMe} /$ hexanes $)$ to yield the product as a colourless oil $(21 \mathrm{mg}, 0.087 \mathrm{mmol}, 44 \%)$. ${ }^{\mathbf{1}} \mathbf{H} \mathbf{~ N M R}$ $\left(400 \mathrm{MHz}, \mathrm{CDCl}_{3}, 298 \mathrm{~K}\right): \delta_{\mathrm{H}} 7.24-7.15(\mathrm{~m}, 4 \mathrm{H}), 7.01-6.93(\mathrm{~m}, 2 \mathrm{H}), 6.88-6.82(\mathrm{~m}, 2 \mathrm{H}), 3.81$ (s, 3H), 1.34-1.20 (m, 4H) ppm; ${ }^{19} \mathbf{F}$ NMR $\left(376 \mathrm{MHz}, \mathrm{CDCl}_{3}, 298 \mathrm{~K}\right): \delta_{\mathrm{F}}-117.5 ;{ }^{13} \mathbf{C ~ N M R}$ $\left(100 \mathrm{MHz}, \mathrm{CDCl}_{3}, 298 \mathrm{~K}\right): \delta_{\mathrm{C}} 161.1(\mathrm{~d}, J=242.8 \mathrm{~Hz}), 157.9,141.9,137.7,129.7(\mathrm{~d}, J=7.9 \mathrm{~Hz})$, 129.5, $114.9(\mathrm{~d}, J=21.1 \mathrm{~Hz}), 113.7,55.3,28.7,16.2 \mathrm{ppm}$; HRMS $\mathrm{m} / z$ (DART): calcd for $\mathrm{C}_{16} \mathrm{H}_{16} \mathrm{OF}(\mathrm{M}+\mathrm{H}): 243.1180$; found: 243.1184; IR (neat): 3079, 3004, 2947, 2836, 2005, 1606, $1509,1460,1243,1218,1030,822,554 \mathrm{~cm}^{-1} ; \mathbf{R}_{\mathbf{f}}(40 \% \mathrm{PhMe} /$ hexanes; UV/p-anisaldehyde): 0.58 .<smiles>COc1ccc(C2(c3ccc(-c4ccccc4)cc3)CC2)cc1</smiles>

4-(1-(4-Methoxyphenyl)cyclopropyl)-1,1'-biphenyl (3af): Prepared on 0.20-mmol scale. The crude residue was purified by flash column chromatography (gradient of $0-40 \% \mathrm{PhMe} /$ hexanes) to yield the product as a white solid (26 mg, $0.087 \mathrm{mmol}, 44 \%) .{ }^{1} \mathbf{H} \mathbf{~ N M R}\left(500 \mathrm{MHz}, \mathrm{CDCl}_{3}\right.$, $298 \mathrm{~K}): \delta_{\mathrm{H}} 7.58-7.53(\mathrm{~m}, 2 \mathrm{H}), 7.50-7.45(\mathrm{~m}, 2 \mathrm{H}), 7.44-7.38(\mathrm{~m}, 2 \mathrm{H}), 7.34-7.29(\mathrm{~m}, 1 \mathrm{H}), 7.26-$ $7.22(\mathrm{~m}, 4 \mathrm{H}), 6.87-6.82(\mathrm{~m}, 2 \mathrm{H}), 3.80(\mathrm{~s}, 3 \mathrm{H}), 1.33-1.27(\mathrm{~m}, 4 \mathrm{H}) \mathrm{ppm} ;{ }^{13} \mathbf{C}$ NMR $(125 \mathrm{MHz}$, $\left.\mathrm{CDCl}_{3}, 298 \mathrm{~K}\right): \delta_{\mathrm{C}} 158.1,145.7,141.1,138.8,137.7,130.2,128.8,128.3,127.2,127.1,127.1$, 113.9, 55.4, 29.1, 16.6 ppm; HRMS m/z (DART): calcd for $\mathrm{C}_{22} \mathrm{H}_{24} \mathrm{NO}\left(\mathrm{M}+\mathrm{NH}_{4}\right)$ : 318.1852; found: 318.1855; IR (neat): 3031, 2951, 2931, 2005, 1611, 1511, 1486, 1451, 1242, 1181, 1174, 1037, 827, $761 \mathrm{~cm}^{-1}$; m.p.: $48-50{ }^{\circ} \mathrm{C}$; $\mathbf{R}_{\mathbf{f}}(40 \% \mathrm{PhMe} /$ hexanes; UV/p-anisaldehyde): 0.55.<smiles>COc1ccc(C2(c3ccc(C(C)(C)C)cc3)CC2)cc1</smiles>

1-(tert-Butyl)-4-(1-(4-methoxyphenyl)cyclopropyl)benzene (3ag): Prepared on 0.20-mmol scale. The crude residue was purified by flash column chromatography (gradient of $0-60 \%$ $\mathrm{PhMe} /$ hexanes) to yield the product as a white solid (25 mg, $0.089 \mathrm{mmol}, 45 \%) .{ }^{\mathbf{1}} \mathbf{H}$ NMR (500 $\left.\mathrm{MHz}, \mathrm{CDCl}_{3}, 298 \mathrm{~K}\right): \delta_{\mathrm{H}} 7.30-7.26(\mathrm{~m}, 2 \mathrm{H}), 7.25-7.21(\mathrm{~m}, 2 \mathrm{H}), 7.13-7.09(\mathrm{~m}, 2 \mathrm{H}), 6.86-8.81$ (m, 2H), 3.80 (s, 3H), 1.30 (s, 9H), 1.28-1.23 (m, 4H) ppm; ${ }^{13}$ C NMR (125 MHz, CDCl, 298 $\mathrm{K}): \delta_{\mathrm{C}} 158.0,148.6,143.4,138.1,130.2,127.4,125.2$, 113.8, 55.4, 34.4, 31.5, 28.8, 16.5 ppm; HRMS $m / z$ (DART): calcd for $\mathrm{C}_{20} \mathrm{H}_{28} \mathrm{NO}\left(\mathrm{M}+\mathrm{NH}_{4}\right)$ : 298.2165; found: 298.2163; IR (neat): 
2959, 2903, 1608, 1511, 1440, 1290, 1242, 1169, 1025, 1017, 832, 816, $577 \mathrm{~cm}^{-1}$; m.p.: $75-77$ ${ }^{\circ} \mathrm{C} ; \mathbf{R}_{\mathbf{f}}(40 \% \mathrm{PhMe} /$ hexanes; UV/KMnO 4$): 0.60$.

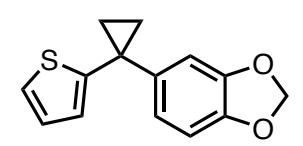

5-(1-(Thiophen-2-yl)cyclopropyl)benzo[1,3]dioxole (3ah): Prepared on 0.20-mmol scale. The crude residue was purified by flash column chromatography (gradient of $0-40 \% \mathrm{PhMe} / \mathrm{hexanes}$ ) to yield the product as a colourless oil $(16 \mathrm{mg}, 0.066 \mathrm{mmol}, 33 \%) .{ }^{1} \mathbf{H} \mathbf{~ N M R}\left(400 \mathrm{MHz}, \mathrm{CDCl}_{3}\right.$, $298 \mathrm{~K}): \delta_{\mathrm{H}} 7.06(\mathrm{dd}, J=5.2,1.3 \mathrm{~Hz}, 1 \mathrm{H}), 6.88-6.83(\mathrm{~m}, 3 \mathrm{H}), 6.76-6.72(\mathrm{~m}, 1 \mathrm{H}), 6.69(\mathrm{~d}, J=3.5$, $1.2 \mathrm{~Hz}, 1 \mathrm{H}), 5.93(\mathrm{~s}, 2 \mathrm{H}), 1.34-1.29(\mathrm{~m}, 4 \mathrm{H}) \mathrm{ppm} ;{ }^{13} \mathbf{C} \mathbf{N M R}\left(100 \mathrm{MHz}, \mathrm{CDCl}_{3}, 298 \mathrm{~K}\right): \delta_{\mathrm{C}}$ 152.3, 147.6, 146.3, 139.0, 126.7, 123.8, 123.2, 122.2, 109.9, 108.1, 101.1, 26.4, 18.4 ppm;

HRMS $m / z$ (DART): calcd for $\mathrm{C}_{14} \mathrm{H}_{13} \mathrm{O}_{2} \mathrm{~S}: 245.0631$; found: 245.0638; IR (neat): 3077, 3004, 2887, 1503, 1485, 1435, 1226, 1035, 932, 809, $692 \mathrm{~cm}^{-1}$; $\mathbf{R}_{\mathbf{f}}(40 \% \mathrm{PhMe} /$ hexanes; UV/panisaldehyde): 0.53 .

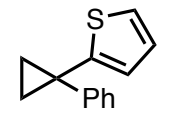

2-(1-Phenylcyclopropyl)thiophene (3ai): Prepared on $0.20-\mathrm{mmol}$ scale. The crude residue was purified by flash column chromatography (gradient of $0-20 \% \mathrm{PhMe} /$ hexanes) to yield the product as a colourless oil $(17 \mathrm{mg}, 0.085 \mathrm{mmol}, 43 \%) .{ }^{1} \mathbf{H} \mathbf{~ N M R}\left(500 \mathrm{MHz}, \mathrm{CDCl}_{3}, 298 \mathrm{~K}\right): \delta_{\mathrm{H}}$ 7.36-7.32 (m, 2H), 7.32-7.27 (m, 2H), 7.24-7.19 (m, 1H), $7.08(\mathrm{dd}, J=5.2,1.3 \mathrm{~Hz}, 1 \mathrm{H}), 6.86$ $(\mathrm{dd}, J=5.2,3.5 \mathrm{~Hz}, 1 \mathrm{H}), 6.72(\mathrm{dd}, J=3.6,1.3 \mathrm{~Hz}, 1 \mathrm{H}), 1.39-1.35(\mathrm{~m}, 4 \mathrm{H}) \mathrm{ppm} ;{ }^{13} \mathbf{C} \mathbf{N M R}(125$ $\left.\mathrm{MHz}, \mathrm{CDCl}_{3}, 298 \mathrm{~K}\right): \delta_{\mathrm{C}} 151.8,145.0,128.7,128.5,126.7,126.6,124.4,123.3,26.2,18.4$ ppm; HRMS $m / z$ (DART): calcd for $\mathrm{C}_{13} \mathrm{H}_{13} \mathrm{~S}(\mathrm{M}+\mathrm{H})$ : 201.0733; found: 201.0738; IR (neat): 3084, $3030,1599,1494,1444,1221,1029,850,738 \mathrm{~cm}^{-1}$. 


\section{Preparation of arylzinc reagents}

\section{D.1. Procedure for preparation of arylzinc reagents}

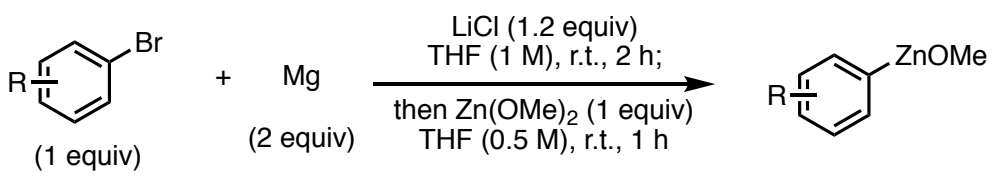

General Procedure C: Preparation of arylzinc reagents

Preparation of the Grignard reagent (representative procedure on 6.0-mmol scale): To a flamedried 16-mL culture tube with stir bar was added magnesium( 0$)$ turnings $(0.29 \mathrm{~g}, 12 \mathrm{mmol}, 2.0$ equiv) and lithium chloride ( $0.30 \mathrm{~g}, 7.2 \mathrm{mmol}, 1.2$ equiv). The tube was sealed with a size 19 septum and electrical tape and was flame-dried under vacuum and cooled under $\mathrm{N}_{2}$. THF (6.0 $\mathrm{mL}, 1.0 \mathrm{M}$ ) was added. The tube was equipped with syringes containing 1,2-dibromoethane (ca. $0.010 \mathrm{~mL}, 0.12 \mathrm{mmol}, 0.020$ equiv) and the desired aryl bromide ( $6.0 \mathrm{mmol}, 1.0$ equiv). (If the aryl bromide was solid, the substrate was prepared as a stock solution in $2.0 \mathrm{~mL}$ of THF and 4.0 $\mathrm{mL}$ of THF as reaction solvent was used instead of $6.0 \mathrm{~mL}$.) The magnesium was activated with 1,2-dibromoethane and the aryl bromide was added portionwise over $1 \mathrm{~h}$ at r.t. The reaction was stirred for an additional $1 \mathrm{~h}$ at r.t. to yield the Grignard reagent as a grey-black solution.

Preparation of $\mathrm{Zn}(\mathrm{OMe}) 2:{ }^{1} \mathrm{~A}$ flame-dried $16-\mathrm{mL}$ culture tube (or appropriately sized flask) with a stir bar was sealed with a size-19 septum and brought into a glovebox. Zinc(II) chloride ( 0.82 g, $6.0 \mathrm{mmol}, 1.0$ equiv) and lithium methoxide $(0.46 \mathrm{~g}, 12 \mathrm{mmol}, 2.0$ equiv) were added and the tube was again sealed with the size-19 septum and electrical tape and was removed from the glovebox. THF $(6.0 \mathrm{~mL}, 1 \mathrm{M})$ was added and the reaction was stirred at r.t. for $1 \mathrm{~h}$ (sonication or shaking may initially be necessary) to yield a $1 \mathrm{M}$ solution of $\mathrm{Zn}(\mathrm{OMe})_{2}$ in THF as a cloudy-grey solution.

Preparation of the arylzinc reagent: While stirring the solution of $\mathrm{Zn}(\mathrm{OMe})_{2}$ in THF at r.t., the solution of Grignard reagent was transferred to the solution of $\mathrm{Zn}(\mathrm{OMe})_{2}$ using a syringe. After addition, the solution was stirred for $1 \mathrm{~h}$ at r.t. to yield the arylzinc reagent as a grey solution. The reagent was stored at r.t. under $\mathrm{N}_{2}$. For best reproducibility, the reagent was aged before use for $72 \mathrm{~h}$ at r.t. under $\mathrm{N}_{2}$ to allow precipitate to settle to the bottom of the flask (Figure S3).

Arylzinc reagents with other counterions (Table S6) were prepared using the same protocol and by transmetallating with the appropriate $\mathrm{Zn}$ (II) source.

Titration of arylzinc reagents using $\mathrm{I}_{2}:{ }^{3}$ To an $8-\mathrm{mL}$ threaded culture tube with a stir bar was added $\mathrm{LiCl}$ (ca. $21 \mathrm{mg}, 0.50 \mathrm{mmol}$ ) and the tube was flame-dried under vacuum and cooled under $\mathrm{N}_{2}$. The tube was briefly opened to air and $\mathrm{I}_{2}$ (ca. $25-50 \mathrm{mg}, 0.10-0.20 \mathrm{mmol}$ ) was added. The exact amount of $I_{2}$ was recorded. The tube was evacuated and backfilled with $\mathrm{N}_{2}(\times 3)$ and THF $(2.0 \mathrm{~mL})$ was added. The solution was cooled to $0{ }^{\circ} \mathrm{C}$ and arylzinc reagent was added dropwise to the solution. The volume of arylzinc reagent required to turn the solution colourless was used to calculate the titre of the arylzinc reagent, which was generally $0.30-0.40 \mathrm{M}$. 
Table S9. Scale and concentration of arylzinc reagents prepared for this study<smiles>C[14CH2]c1ccc(OC)cc1</smiles>

20-mmol scale

[0.30-0.40 M]<smiles>COc1ccc([Ge]c2ccc(S(C)(=O)=O)cc2)cc1</smiles>

4.0-mmol scale

$[0.21 \mathrm{M}]$<smiles>COc1ccc([Ge]c2ccc(OC)cc2)cc1</smiles>

4.0-mmol scale $[0.30 \mathrm{M}]$<smiles>COc1ccc(OCc2ccccc2)cc1</smiles>

6.0-mmol scale [0.20 M]<smiles>COc1ccc([Ge]Cl)cc1</smiles>

20-mmol scale [0.44 M]<smiles>COc1ccc([Ge]OC(C)=O)cc1OC</smiles>

4.0-mmol scale [0.20 M]<smiles>CO[Ga]c1ccc(N(Cc2ccccc2)Cc2ccccc2)cc1</smiles>

4.0-mmol scale [0.21 M]<smiles>CO[Ga]c1ccc2c3ccccc3n(C)c2c1</smiles>

2.0-mmol scale [0.27 M]<smiles>CO[14C](=O)c1cnc(N2CCN(Cc3ccccc3)CC2)nc1</smiles><smiles>CO[Ga]c1ccc(O[C@@H]2C[C@H](C)CC[C@H]2C(C)C)cc1</smiles>

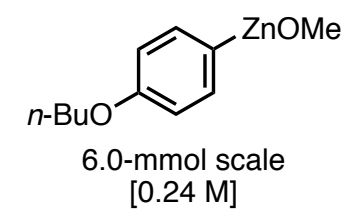<smiles>C[C+](C)c1ccc2c(c1)OCO2</smiles>

6.0-mmol scale [0.32 M]<smiles>COCc1ccc(Oc2ccccc2)cc1</smiles>

4.0-mmol scale [0.28 M]<smiles>COC(C)c1ccc(C(C)(C)C)cc1</smiles>

6.0-mmol scale [0.34 M]<smiles>Fc1ccc([Ge]Cl)cc1</smiles>

\section{0-mmol scale} [0.47 M]<smiles>Cl[Ge]c1ccc2ccccc2c1</smiles>

4.0-mmol scale [0.24 M]<smiles>Cl[Ge]c1ccc2sccc2c1</smiles>

2.0-mmol scale [0.39 M]<smiles>Cl[Ge]c1ccsc1</smiles>

4.0-mmol scale [0.36 M]<smiles>Cl[Ge]c1ccc2oc3ccccc3c2c1</smiles>

2.0-mmol scale [0.30 M]

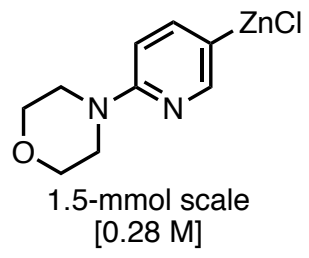
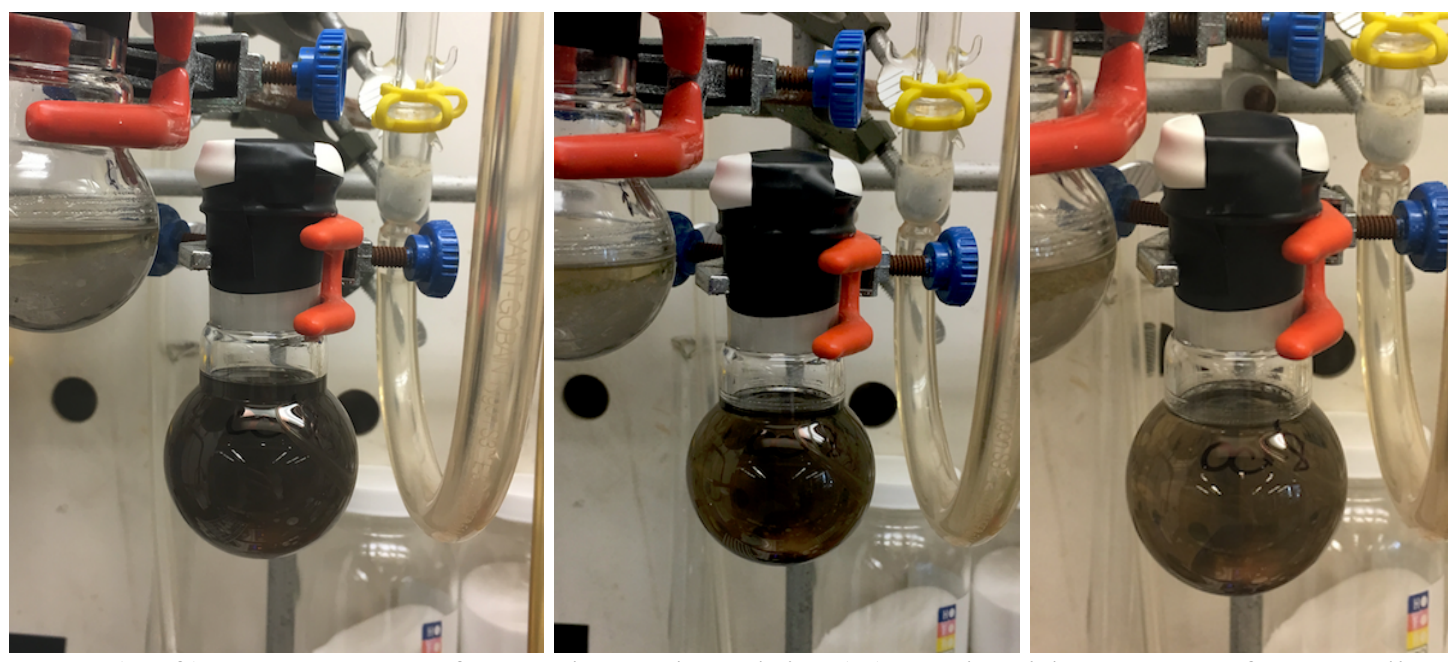

Figure S3. (Left) Appearance of 4-methoxyphenylzinc(II) methoxide reagent after standing for $1 \mathrm{~h}$ at r.t.; (Centre) Appearance after 24 h; (Right) Appearance after 72 h. 


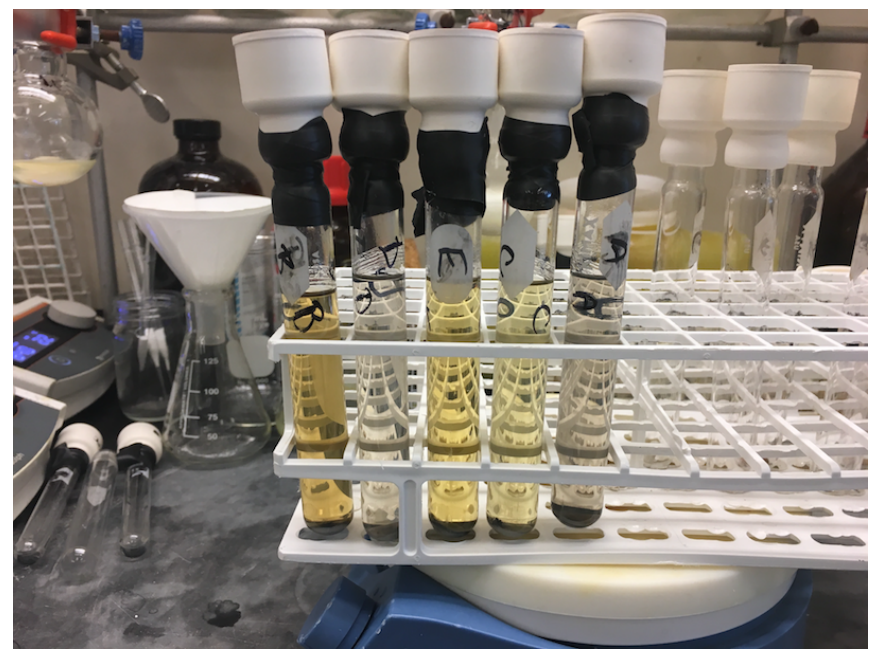

Figure S4. Typical appearance of arylzinc reagents after standing for $72 \mathrm{~h}$ at r.t. 


\section{D.2. Preparation of aryl bromide starting materials}

The following aryl bromides were prepared as previously described: ${ }^{4} 1$-bromo-4isopropoxybenzene, 1-bromo-4-phenoxybenzene, 1-bromo-4-(((1R,2S,5R)-2-isopropyl-5methylcyclohexyl)oxy)benzene, $N, N$-dibenzyl-4-bromoaniline, (4-bromophenoxy)(tertbutyl)dimethylsilane, 2-(4-benzylpiperazin-1-yl)-5-bromopyrimidine, 2-bromodibenzofuran.

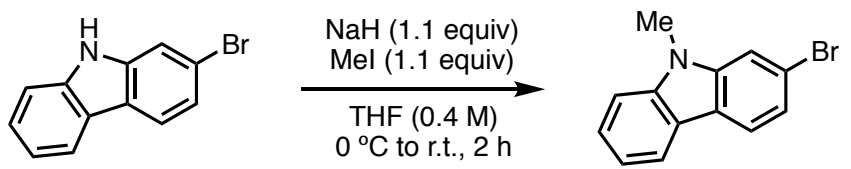

2-Bromo-9-methyl-9H-carbazole (S1): To a 50-mL flask with a stir bar were added 2bromocarbazole (1.2 g, $5.0 \mathrm{mmol}, 1.0$ equiv) and THF (12 mL, $0.40 \mathrm{M})$, and the solution was cooled to $0{ }^{\circ} \mathrm{C}$. Sodium hydride $(0.22 \mathrm{~g}, 5.5 \mathrm{mmol}, 1.1$ equiv) was added at once and the reaction was stirred at r.t. for $30 \mathrm{~min}$. Iodomethane $(0.34 \mathrm{~mL}, 5.5 \mathrm{mmol}, 1.1$ equiv) was added and the reaction was stirred at r.t. for $2 \mathrm{~h}$. The reaction was quenched with sat. aq. $\mathrm{NH}_{4} \mathrm{Cl}$ and extracted with EtOAc $(\times 3)$, and the organic fractions were combined, washed with brine $(\times 1)$, dried over $\mathrm{MgSO}_{4}$, and concentrated. The solid concentrate was washed with hexanes $(5.0 \mathrm{~mL})$ to yield the product as an off-white solid (1.2 g, $4.6 \mathrm{mmol}, 92 \%) .{ }^{1} \mathbf{H} \mathbf{~ N M R}\left(400 \mathrm{MHz}, \mathrm{CDCl}_{3}, 298 \mathrm{~K}\right)$ : $\delta_{\mathrm{H}}$ 8.08-8.03 (m, 1H), 7.94-7.91 (m, 1H), 7.56-7.48 (m, 2H), 7.39 (dt, $J=8.2,0.9 \mathrm{~Hz}, 1 \mathrm{H}), 7.34$ $(\mathrm{dd}, J=8.2,1.7 \mathrm{~Hz}, 1 \mathrm{H}), 7.26(\mathrm{td}, J=7.7,1.0 \mathrm{~Hz}, 1 \mathrm{H}), 3.80(\mathrm{~s}, 3 \mathrm{H}) \mathrm{ppm} ;{ }^{13} \mathbf{C}$ NMR $(100 \mathrm{MHz}$, $\left.\mathrm{CDCl}_{3}, 298 \mathrm{~K}\right): \delta_{\mathrm{C}} 141.9,141.2,126.3,122.4,122.1,121.9,121.5,120.4,119.6,119.4,111.7$, 108.8, 29.3 ppm; HRMS $m / z$ (DART): calcd for $\mathrm{C}_{13} \mathrm{H}_{11} \mathrm{NBr}(\mathrm{M}+\mathrm{H}): 260.0069$; found: 260.0070; IR (neat): 2926, 2853, 1590, 1476, 1448, 1320, 1245, 1127, 1054, 842, 797, 742, $720 \mathrm{~cm}^{-1}$; m.p.: $74-75^{\circ} \mathrm{C} ; \mathbf{R}_{\mathbf{f}}(9: 1$ hexanes/EtOAc; UV): 0.59 .

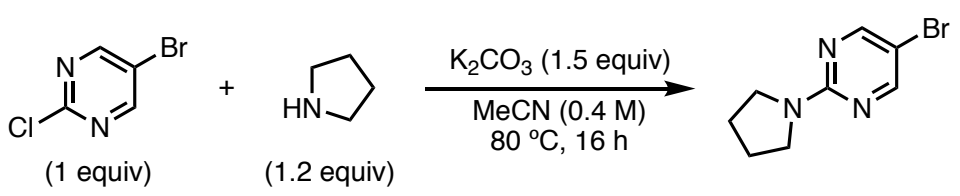

5-Bromo-2-(pyrrolidin-1-yl)pyrimidine (S2): To a 50-mL flask with a stir bar were added 5bromo-2-chloropyrimidine ( $1.9 \mathrm{~g}, 10 \mathrm{mmol}, 1.0$ equiv), potassium carbonate $(2.1 \mathrm{~g}, 15 \mathrm{mmol}$, 1.5 equiv), $\mathrm{MeCN}(25 \mathrm{~mL}, 0.40 \mathrm{M}$ ), and piperidine (1.0 mL, $12 \mathrm{mmol}, 1.2$ equiv), and the reaction was stirred at $80{ }^{\circ} \mathrm{C}$ for $16 \mathrm{~h}$. The reaction was cooled to r.t., quenched with $\mathrm{H}_{2} \mathrm{O}$, and extracted with EtOAc $(\times 3)$. The organic fractions were combined, washed with brine $(\times 1)$, dried over $\mathrm{MgSO}_{4}$, and concentrated. The solid concentrate was washed with hexanes $(5.0 \mathrm{~mL})$ to yield the product as an off-white solid $(2.0 \mathrm{~g}, 8.8 \mathrm{mmol}, 88 \%) .{ }^{\mathbf{1}} \mathbf{H} \mathbf{~ N M R}\left(400 \mathrm{MHz}, \mathrm{CDCl}_{3}, 298\right.$ $\mathrm{K}): \delta_{\mathrm{H}} 8.28(\mathrm{~s}, 2 \mathrm{H}), 3.57-3.46(\mathrm{~m}, 4 \mathrm{H}), 2.04-1.93(\mathrm{~m}, 4 \mathrm{H}) \mathrm{ppm} ;{ }^{13} \mathbf{C} \mathbf{N M R}\left(100 \mathrm{MHz}, \mathrm{CDCl}_{3}\right.$, $298 \mathrm{~K}$ ): $\delta_{\mathrm{C}} 158.6,158.0,105.0,47.0,25.7 \mathrm{ppm}$; HRMS $\mathrm{m} / z$ (DART): calcd for $\mathrm{C}_{8} \mathrm{H}_{11} \mathrm{~N}_{3} \mathrm{Br}$ $(\mathrm{M}+\mathrm{H}): 228.0131$; found: 228.0135; IR (neat): 2971, 2872, 1578, 1516, 1450, 1283, 1153, 1115 , 939, 782, $639 \mathrm{~cm}^{-1}$; m.p.: $89-91^{\circ} \mathrm{C}$; $\mathbf{R}_{\mathbf{f}}(6: 4$ hexanes/EtOAc; UV): 0.81.

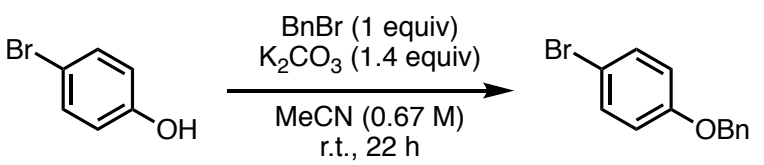

1-(Benzyloxy)-4-bromobenzene (S3): To a flame-dried 100-mL flask with a stir bar was added 1-Bromo-4-iodobenzene (5.2 g, $30 \mathrm{mmol}, 1.0$ equiv). The flask was evacuated and backfilled 
with $\mathrm{N}_{2}(\times 3)$ and $\mathrm{MeCN}(75 \mathrm{~mL}, 0.40 \mathrm{M})$ was added. Potassium bicarbonate $(5.8 \mathrm{~g}, 42 \mathrm{mmol}$, 1.4 equiv) was added and the solution was stirred at r.t. for 15 minutes. Benzyl bromide $(3.6 \mathrm{~mL}$, $30 \mathrm{mmol}, 1.0$ equiv) was added and the reaction was stirred at r.t. for 22 hours. The reaction was then opened to air and filtered through celite. The filtrate was concentrated under vacuum and purified by flash column chromatography on silica gel (gradient of $0-5 \%$ EtOAc/hexanes). The fractions containing desired product were collected, combined, and concentrated to yield the desired product as a white solid (7.4 g, $28 \mathrm{mmol}, 94 \%)$. Analytical data: ${ }^{4} \mathbf{H}$ NMR (400 MHz, $\left.\mathrm{CDCl}_{3}, 298 \mathrm{~K}\right): \delta_{\mathrm{H}} 7.43-7.39(\mathrm{~m}, 3 \mathrm{H}), 7.39-7.36(\mathrm{~m}, 3 \mathrm{H}), 7.35-7.30(\mathrm{~m}, 1 \mathrm{H}), 6.89-6.82(\mathrm{~m}$, 2H), $5.04(\mathrm{~s}, 2 \mathrm{H}) \mathrm{ppm} ;{ }^{13} \mathbf{C} \mathbf{N M R}\left(100 \mathrm{MHz}, \mathrm{CDCl}_{3}, 298 \mathrm{~K}\right): \delta_{\mathrm{C}} 157.9,136.6,132.3,128.7$, 128.1, 127.4, 116.7, 113.1, 70.3 ppm; $\mathbf{R}_{\mathbf{f}}$ (95:5 hexanes/EtOAc; vanillin): 0.73.

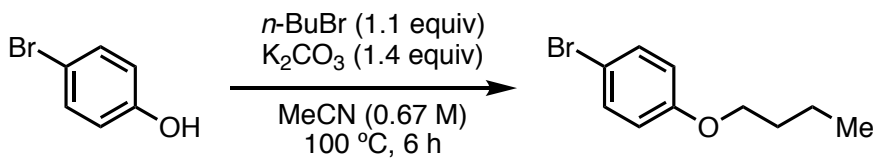

1-Bromo-4-butoxybenzene (S4): To a flame-dried 100-mL flask with a stir bar was added 4bromophenol ( $6.9 \mathrm{~g}, 40 \mathrm{mmol}, 1.0$ equiv). The flask was set up with a reflux condenser, was evacuated and backfilled with $\mathrm{N}_{2}(\times 3)$ and $\mathrm{MeCN}(60 \mathrm{~mL}, 0.67 \mathrm{M})$ was added. Potassium bicarbonate $(7.7 \mathrm{~g}, 56 \mathrm{mmol}, 1.4$ equiv) was added and the solution was stirred at r.t. for 10 minutes. 1-Bromobutane $(4.7 \mathrm{~mL}, 44 \mathrm{mmol}, 1.1$ equiv) was added and the reaction was stirred at $100{ }^{\circ} \mathrm{C}$ for 6 hours. The reaction was allowed to cool to r.t., opened to air and then filtered. The filtrate was concentrated under vacuum and purified by flash column chromatography on silica gel (gradient of $0-5 \%$ EtOAc/hexanes). The fractions containing desired product were collected, combined, and concentrated to yield the desired product as a colourless oil $(7.8 \mathrm{~g}, 34 \mathrm{mmol}$, 85\%). Analytical data: ${ }^{1} \mathbf{H}$ NMR $\left(400 \mathrm{MHz}, \mathrm{CDCl}_{3}, 298 \mathrm{~K}\right): \delta_{\mathrm{H}} 7.40-7.31(\mathrm{~m}, 2 \mathrm{H}), 6.81-6.73$ (m, 2H), $3.92(\mathrm{t}, J=6.5 \mathrm{~Hz}, 2 \mathrm{H}), 1.75$ (ddt, $J=8.9,7.8,6.3 \mathrm{~Hz}, 2 \mathrm{H}), 1.54-1.41(\mathrm{~m}, 2 \mathrm{H}), 0.97(\mathrm{t}$, $J=7.4 \mathrm{~Hz}, 3 \mathrm{H}) \mathrm{ppm} ;{ }^{13} \mathbf{C} \mathbf{N M R}\left(100 \mathrm{MHz}, \mathrm{CDCl}_{3}, 298 \mathrm{~K}\right): \delta_{\mathrm{C}} 158.4,132.3,116.5,112.7,68.1$, 31.4, 19.3, 14.0 ppm; $\mathbf{R}_{\mathbf{f}}\left(9: 1\right.$ hexanes/EtOAc; $\left.\mathrm{KMnO}_{4}\right): 0.87$.

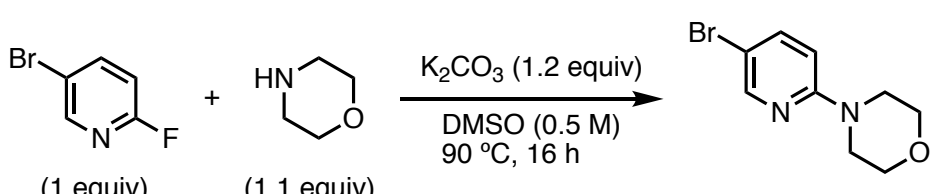

4-(5-Bromopyridin-2-yl)morpholine (S5): To a 25-mL flask with a stir bar were added potassium carbonate $(0.83 \mathrm{~g}, 6.0 \mathrm{mmol}, 1.2$ equiv), DMSO $(10 \mathrm{~mL}, 0.50 \mathrm{M}), 5$-bromo-2fluoropyridine $(0.51 \mathrm{~mL}, 5.0 \mathrm{mmol}, 1.0$ equiv), and morpholine $(0.48 \mathrm{~mL}, 5.5 \mathrm{mmol}, 1.1 \mathrm{equiv})$, and the reaction was stirred at $90^{\circ} \mathrm{C}$ for $16 \mathrm{~h}$. The reaction was cooled to r.t., quenched with sat. aq. $\mathrm{NH}_{4} \mathrm{Cl}$, and extracted with EtOAc $(\times 3)$, and the organic fractions were washed with brine $(\times 1)$, dried over $\mathrm{MgSO}_{4}$, and concentrated. The concentrate was triturated with hexanes and dried under high vacuum to yield the product as an off-white solid (1.0 g, $4.1 \mathrm{mmol}, 82 \%)$. The analytical data was consistent with literature. ${ }^{5}$ 


\section{E. Preparation of cyclopropanol starting materials}

E.1. Preparation of cyclopropanols with alternate leaving groups

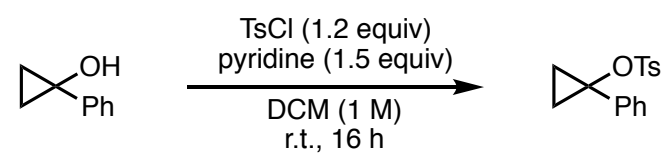

1-Phenylcyclopropyl 4-methylbenzenesulfonate (1a): To a 100-mL round-bottom flask with a stir bar were sequentially added 1-phenylcyclopropanol (3.1 g, $23 \mathrm{mmol}, 1.0$ equiv), DCM (23 mL, 1.0 M), pyridine ( $2.8 \mathrm{~mL}, 35 \mathrm{mmol}, 1.5$ equiv), and tosyl chloride (5.3 g, $28 \mathrm{mmol}, 1.2$ equiv). The reaction was stirred at r.t. for $16 \mathrm{~h}$. The reaction was quenched with $\mathrm{H}_{2} \mathrm{O}$ and extracted with DCM $(\times 3)$, and the organic fractions were combined, washed with brine $(\times 1)$, dried over $\mathrm{MgSO}_{4}$, and concentrated. The concentrate was dried under high vacuum at $0{ }^{\circ} \mathrm{C}$ to induce solidification of the crude residue. The product was reprecipitated (pyridine $/ \mathrm{H}_{2} \mathrm{O}$ ) and the precipitate was washed with hexanes $(3 \times 20 \mathrm{~mL})$ and dried under high vacuum to yield the product as a white solid ( $3.2 \mathrm{~g}, 11 \mathrm{mmol}, 48 \%)$. Analytical data: ${ }^{1} \mathbf{H}$ NMR $\left(400 \mathrm{MHz}, \mathrm{CDCl}_{3}\right.$, $298 \mathrm{~K}): \delta_{\mathrm{H}} 7.52-7.44(\mathrm{~m}, 2 \mathrm{H}), 7.33-7.26(\mathrm{~m}, 2 \mathrm{H}), 7.22-7.15(\mathrm{~m}, 3 \mathrm{H}), 7.13-7.07$ (m, 2H), 2.36 (s, 3H), 1.66-1.56 (m, 2H), 1.18-1.11 (m, 2H) ppm; ${ }^{13} \mathbf{C}$ NMR (100 MHz, CDCl 3 , $\left.298 \mathrm{~K}\right): \delta_{\mathrm{C}}$ $144.2,137.8,135.2,129.4,128.2,128.2,128.2,127.8,67.2,21.7,13.7 \mathrm{ppm} ; \mathbf{R}_{\mathbf{f}}(5 \%$

EtOAc/hexanes; UV): 0.40 .

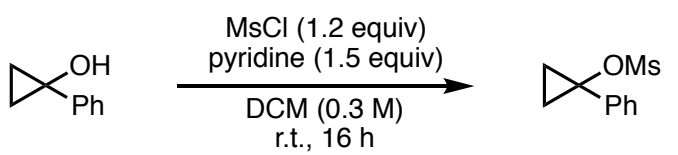

1-Phenylcyclopropyl methanesulfonate (1b): To a $25-\mathrm{mL}$ flask with a stir bar were added 1phenylcyclopropanol $(0.40 \mathrm{~g}, 3.0 \mathrm{mmol}, 1.0$ equiv), DCM $(10 \mathrm{~mL}, 0.30 \mathrm{M})$, pyridine $(0.36 \mathrm{~mL}$, $4.5 \mathrm{mmol}, 1.5$ equiv), and mesyl chloride $(0.28 \mathrm{~mL}, 3.6 \mathrm{mmol}, 1.2$ equiv), and the reaction was stirred at r.t. for $16 \mathrm{~h}$. The reaction was concentrated and purified by flash column chromatography (gradient of 10-40\% EtOAc/hexanes) to yield the product as a colourless oil $(0.47 \mathrm{~g}, 2.2 \mathrm{mmol}, 73 \%)$. ${ }^{1} \mathbf{H}$ NMR $\left(500 \mathrm{MHz}, \mathrm{CDCl}_{3}, 298 \mathrm{~K}\right): \delta_{\mathrm{H}} 7.59-7.55(\mathrm{~m}, 2 \mathrm{H}), 7.42-7.34$ $(\mathrm{m}, 3 \mathrm{H}), 2.54(\mathrm{~s}, 3 \mathrm{H}), 1.69-1.64(\mathrm{~m}, 2 \mathrm{H}), 1.24-1.18(\mathrm{~m}, 2 \mathrm{H}) \mathrm{ppm} ;{ }^{13} \mathbf{C}$ NMR $\left(125 \mathrm{MHz}, \mathrm{CDCl}_{3}\right.$, $298 \mathrm{~K}): \delta_{\mathrm{C}} 137.4,129.2,129.1,128.9,67.5,39.8,13.5 \mathrm{ppm}$; HRMS $\mathrm{m} / z$ (DART): calcd for $\mathrm{C}_{10} \mathrm{H}_{16} \mathrm{NO}_{3} \mathrm{~S}\left(\mathrm{M}+\mathrm{NH}_{4}\right): 230.0845$; found: 230.0849 ; IR (neat): 3031, 1353, 1213, 1166, 1027, 971, 884, 804, $698 \mathrm{~cm}^{-1} ; \mathbf{R}_{\mathbf{f}}$ (9:1 hexanes/EtOAc; UV/p-anisaldehyde): 0.17.

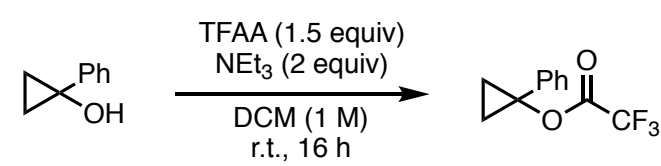

1-Phenylcyclopropyl 2,2,2-trifluoroacetate (1c): To a 50-mL flask with a stir bar were sequentially added 1-phenylcyclopropanol $(1.3 \mathrm{~g}, 10 \mathrm{mmol}, 1.0$ equiv), DCM (10 mL, $1.0 \mathrm{M})$, triethylamine $(2.8 \mathrm{~mL}, 20 \mathrm{mmol}, 1.0$ equiv), and trifluoroacetic anhydride $(2.1 \mathrm{~mL}, 15 \mathrm{mmol}, 1.5$ equiv), and the reaction was stirred at r.t. for $16 \mathrm{~h}$. The reaction was concentrated and purified by flash column chromatography (gradient of $0-4 \%$ EtOAc/hexanes) to yield the product as a colourless oil $(1.2 \mathrm{~g}, 5.0 \mathrm{mmol}, 50 \%)$. ${ }^{1} \mathbf{H}$ NMR $\left(400 \mathrm{MHz}, \mathrm{CDCl}_{3}, 298 \mathrm{~K}\right): \delta_{\mathrm{H}} 7.45-7.28(\mathrm{~m}$, 5H), $1.48-1.29(\mathrm{~m}, 4 \mathrm{H}) \mathrm{ppm} ;{ }^{19} \mathbf{F}$ NMR $\left(376 \mathrm{MHz}, \mathrm{CDCl}_{3}, 298 \mathrm{~K}\right): \delta_{\mathrm{F}}-75.4 \mathrm{ppm} ;{ }^{13} \mathbf{C}$ NMR $\left(125 \mathrm{MHz}, \mathrm{CDCl}_{3}, 298 \mathrm{~K}\right): \delta_{\mathrm{C}} 157.1(\mathrm{q}, J=41.9 \mathrm{~Hz}), 137.2,128.8,128.6,127.3,114.4(\mathrm{q}, J=$ 
284.7 Hz), 64.4, $14.0 \mathrm{ppm}$; HRMS $m / z$ (DART): calcd for $\mathrm{C}_{11} \mathrm{H}_{13} \mathrm{NO}_{2} \mathrm{~F}_{3}\left(\mathrm{M}+\mathrm{NH}_{4}\right): 248.0893$; found: 248.08955; IR (neat): 1791, 1361, 1219, 1138, 1100, 1028, 848, 775, 753, $695 \mathrm{~cm}^{-1} ; \mathbf{R}_{\mathbf{f}}$ (5\% EtOAc/hexanes; UV/p-anisaldehyde): 0.48 .

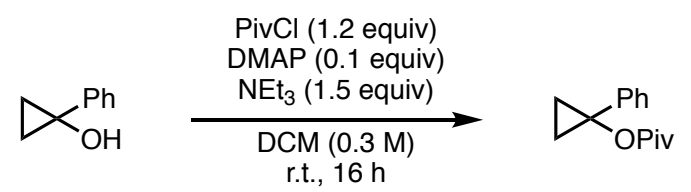

1-Phenylcyclopropyl pivalate (1d): To a $25-\mathrm{mL}$ flask with a stir bar were added 1phenylcyclopropanol $(0.40 \mathrm{~g}, 3.0 \mathrm{mmol}, 1.0$ equiv), DCM $(10 \mathrm{~mL}, 0.30 \mathrm{M})$, triethylamine $(0.63$ $\mathrm{mL}, 4.5 \mathrm{mmol}, 1.5$ equiv), pivaloyl chloride ( $0.44 \mathrm{~mL}, 3.6 \mathrm{mmol}, 1.2$ equiv), and DMAP ( $37 \mathrm{mg}$, $0.30 \mathrm{mmol}, 0.10$ equiv), and the reaction was stirred at r.t. for $16 \mathrm{~h}$. The reaction was quenched with sat. aq. $\mathrm{NH}_{4} \mathrm{Cl}$ and extracted with $\mathrm{DCM}(\times 3)$, and the organic fractions were combined, washed with brine $(\times 1)$, dried over $\mathrm{MgSO}_{4}$, and concentrate. The crude residue was purified by flash column chromatography (gradient of $0-10 \%$ EtOAc/hexanes) to yield the product as a colourless oil (0.48 g, $2.2 \mathrm{mmol}, 73 \%) .{ }^{1} \mathbf{H}$ NMR $\left(400 \mathrm{MHz}, \mathrm{CDCl}_{3}, 298 \mathrm{~K}\right): \delta_{\mathrm{H}} 7.35-7.18$ (m, $5 \mathrm{H}), 1.25-1.21$ (m, 4H), 1.19 (s, 9H) ppm; ${ }^{13} \mathbf{C} \mathbf{N M R}\left(100 \mathrm{MHz}, \mathrm{CDCl}_{3}, 298 \mathrm{~K}\right): \delta_{\mathrm{C}} 178.1$, 140.5, 128.4, 127.1, 125.8, 59.8, 38.7, 27.1, $15.2 \mathrm{ppm}$; HRMS $m / z$ (DART): calcd for $\mathrm{C}_{14} \mathrm{H}_{19} \mathrm{O}_{2}$ (M+H): 219.1380; found: 219.1377; IR (neat): 2975, 1743, 1480, 1456, 1280, 1222, 1136, 1028, $1001,753,695 \mathrm{~cm}^{-1} ; \mathbf{R}_{\mathbf{f}}$ (5\% EtOAc/hexanes; UV/p-anisaldehyde): 0.43 .

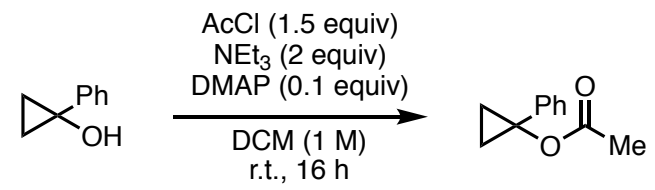

1-Phenylcyclopropyl acetate (S6): To a 25 -mL flask with a stir bar were sequentially added 1phenylcyclopropanol $(0.27 \mathrm{~g}, 2.0 \mathrm{mmol}, 1.0$ equiv), DCM $(2.0 \mathrm{~mL}, 1.0 \mathrm{M})$, acetyl chloride $(0.21$ $\mathrm{mL}, 3.0 \mathrm{mmol}, 1.5$ equiv), triethylamine $(0.56 \mathrm{~mL}, 4.0 \mathrm{mmol}, 2.0$ equiv), and DMAP ( $22 \mathrm{mg}$, $0.20 \mathrm{mmol}, 0.10$ equiv), and the reaction was stirred at r.t. for $16 \mathrm{~h}$. The reaction was quenched with sat. aq. $\mathrm{NH}_{4} \mathrm{Cl}$ and extracted with $\mathrm{DCM}(\times 3)$, and the organic fractions were combined, dried over $\mathrm{MgSO}_{4}$, and concentrated. The crude residue was purified by flash column chromatography (gradient of 5-10\% EtOAc/hexanes) to yield the product as a colourless oil (0.17 g, $0.96 \mathrm{mmol}, 48 \%)$. ${ }^{1} \mathbf{H}$ NMR (500 MHz, $\left.\mathrm{CDCl}_{3}, 298 \mathrm{~K}\right): \delta_{\mathrm{H}} 7.34-7.29(\mathrm{~m}, 4 \mathrm{H}), 7.25-$ $7.21(\mathrm{~m}, 1 \mathrm{H}), 2.04(\mathrm{~s}, 3 \mathrm{H}), 1.31-1.19(\mathrm{~m}, 4 \mathrm{H}) \mathrm{ppm} ;{ }^{13} \mathbf{C} \mathbf{N M R}\left(125 \mathrm{MHz}, \mathrm{CDCl}_{3}, 298 \mathrm{~K}\right): \delta_{\mathrm{C}}$ 170.7, 140.2, 128.4, 127.3, 126.3, 60.1, 21.4, 15.0 ppm; HRMS $\mathrm{m} / z$ (DART): calcd for $\mathrm{C}_{11} \mathrm{H}_{16} \mathrm{NO}_{2}\left(\mathrm{M}+\mathrm{NH}_{4}\right):$ 194.1176; found: 194.1177; IR (neat): 1753, 1499, 1455, 1369, 1244 , $1202,1101,1026,985,754,695 \mathrm{~cm}^{-1} ; \mathbf{R}_{\mathbf{f}}(5 \%$ EtOAc/hexanes; UV/p-anisaldehyde): 0.23 .

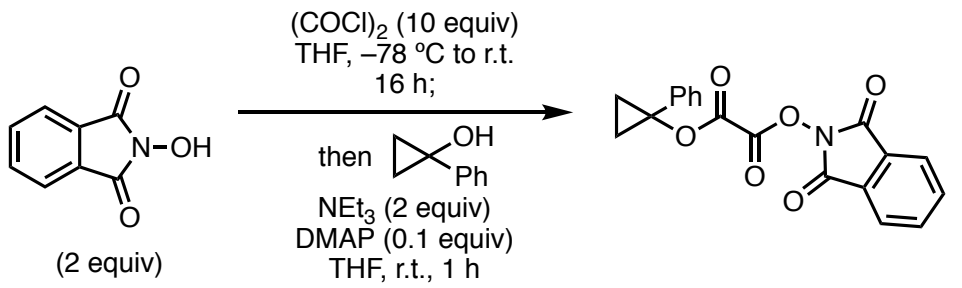

1,3-Dioxoisoindolin-2-yl (1-phenylcyclopropyl) oxalate (1e): ${ }^{7}$ To a flame-dried 500-mL round-bottom flask was added $N$-hydroxyphthalimide $(2.0 \mathrm{~g}, 12 \mathrm{mmol}, 2.0$ equiv). The flask was evacuated and backfilled with $\mathrm{N}_{2}(\times 3)$ and THF $(200 \mathrm{~mL})$ was added. The solution was cooled to 
$-78^{\circ} \mathrm{C}$ and oxalyl chloride (5.1 mL, $60 \mathrm{mmol}, 10$ equiv) was added dropwise. After addition, the flask was removed from the cooling bath and was allowed to warm to r.t. while stirring for $16 \mathrm{~h}$. The solution was concentrated and dried under high vacuum for $1 \mathrm{~h}$ to yield an off-white solid, which was dissolved in THF $(200 \mathrm{~mL})$. To a separate flame-dried 500-mL round-bottom flask with a stir bar were added 1-phenylcyclopropanol $(0.80 \mathrm{~g}, 6.0 \mathrm{mmol}, 1.0$ equiv), triethylamine ( $1.7 \mathrm{~mL}, 12 \mathrm{mmol}, 2.0$ equiv), and DMAP (73 mg, $0.60 \mathrm{mmol}, 0.10$ equiv). While stirring, the THF solution was added by syringe, and the reaction was stirred at r.t. for $1 \mathrm{~h}$. The reaction was concentrated. The concentrate was dissolved in DCM (30 mL), and the solution was poured into hexanes $(1 \mathrm{~L})$. The precipitate was collected by filtration and was washed with hexanes $(\times 1)$, and the filtrate was discarded. The precipitate was extracted with EtOAc $(3 \times 50 \mathrm{~mL})$, and the organic fractions were combined and concentrated. The concentrate was recrystallized (DCM/pentane) to yield the product as a brown solid (1.2 g, $3.4 \mathrm{mmol}, 57 \%)$. 1e was not characterized due to poor solubility in most solvents. However, similar products bearing other substituents have been characterized. ${ }^{7}$

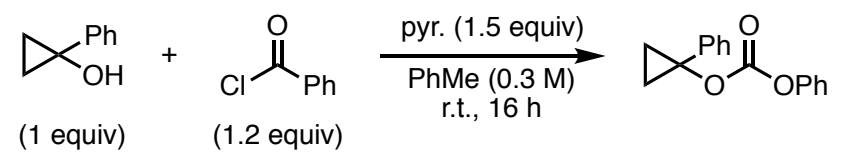

Phenyl (1-phenylcyclopropyl) carbonate (1f): To a flame-dried 50-mL flask with stir bar was added 1-phenylcyclopropanol ( $0.80 \mathrm{~g}, 6.0 \mathrm{mmol}, 1.0 \mathrm{equiv})$. The flask was sealed and evacuated and backfilled with $\mathrm{N}_{2}(\times 3)$ and PhMe $(20 \mathrm{~mL}, 0.30 \mathrm{M})$ was added. Pyridine $(0.73 \mathrm{~mL}, 9.0$ mmol, 1.5 equiv) was added, then phenyl chloroformate $(0.90 \mathrm{~mL}, 7.2 \mathrm{mmol}, 1.2$ equiv) was added dropwise. The reaction was stirred at r.t. under $\mathrm{N}_{2}$ for $16 \mathrm{~h}$. The reaction was quenched with sat. aq. $\mathrm{NH}_{4} \mathrm{Cl}$ and extracted with EtOAc $(\times 3)$. The organic fractions were combined, washed with brine $(\times 1)$, dried over $\mathrm{MgSO}_{4}$, and concentrated. The crude residue was purified by flash column chromatography (gradient of $0-4 \%$ EtOAc/hexanes) to yield the product as a white solid (1.3 g, $5.1 \mathrm{mmol}, 85 \%)$. ${ }^{1} \mathbf{H}$ NMR $\left(500 \mathrm{MHz}, \mathrm{CDCl}_{3}, 298 \mathrm{~K}\right): \delta_{\mathrm{H}} 7.49-7.44(\mathrm{~m}, 2 \mathrm{H}), 7.40$ $7.27(\mathrm{~m}, 5 \mathrm{H}), 7.23-7.17(\mathrm{~m}, 1 \mathrm{H}), 7.16-7.11(\mathrm{~m}, 2 \mathrm{H}), 1.52-1.44(\mathrm{~m}, 2 \mathrm{H}), 1.34-1.23(\mathrm{~m}, 2 \mathrm{H})$ ppm; ${ }^{13} \mathbf{C}$ NMR $\left(125 \mathrm{MHz}, \mathrm{CDCl}_{3}, 298 \mathrm{~K}\right): \delta_{\mathrm{C}} 153.2,151.1,138.9,129.5,128.6,128.0,127.2$, 126.1, 121.1, 63.7, 14.6; HRMS $m / z$ (DART): calcd for $\mathrm{C}_{16} \mathrm{H}_{18} \mathrm{NO}_{3}\left(\mathrm{M}+\mathrm{NH}_{4}\right): 272.1281$; found: 272.1288; IR (neat): 1769, 1593, 1498, 1452, 1247, 1192, 1177, 1098, 1029, 927, 753, 722, 685 $\mathrm{cm}^{-1}$; m.p.: $43-44^{\circ} \mathrm{C}$; $\mathbf{R}_{\mathbf{f}}(5 \%$ EtOAc/hexanes; UV/KMnO 4$): 0.53$.

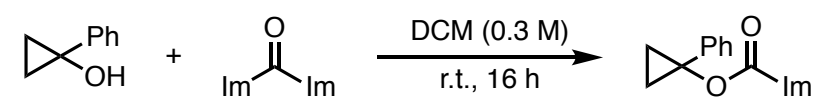

(1 equiv) (1.1 equiv)

1-Phenylcyclopropyl $1 \boldsymbol{H}$-imidazole-1-carboxylate (1g): To a flame-dried 25-mL flask with stir bar was added 1-phenylcyclopropanol ( $0.54 \mathrm{~g}, 4.0 \mathrm{mmol}, 1.0$ equiv). The flask was sealed, evacuated and backfilled with $\mathrm{N}_{2}(\times 3)$, and DCM $(13 \mathrm{~mL}, 0.30 \mathrm{M})$ was added. The flask was briefly opened to air and 1,1'-carbonyldiimidazole $(0.71 \mathrm{~g}, 4.4 \mathrm{mmol}, 1.1$ equiv) was added. The reaction was stirred at r.t. under $\mathrm{N}_{2}$ for $16 \mathrm{~h}$. The reaction was opened to air, dried over $\mathrm{MgSO}_{4}$, and concentrated. The crude residue was purified by flash column chromatography (gradient of 30-40\% EtOAc/hexsnes) to yield the product as a white solid (0.52 g, $2.3 \mathrm{mmol}, 58 \%)$. ${ }^{\mathbf{1}} \mathbf{H}$ NMR $\left(500 \mathrm{MHz}, \mathrm{CDCl}_{3}, 298 \mathrm{~K}\right): 8.16-8.08(\mathrm{~m}, 1 \mathrm{H}), 7.47-7.43(\mathrm{~m}, 2 \mathrm{H}), 7.41-7.38(\mathrm{~m}, 1 \mathrm{H}), 7.38-7.34$ (m, 2H), 7.33-7.28 (m, 1H), 7.09-7.02 (m, 1H), 1.55-1.46 (m, 2H), 1.40-1.32 (m, 2H) ppm; ${ }^{13} \mathbf{C}$ NMR (125 MHz, $\left.\mathrm{CDCl}_{3}, 298 \mathrm{~K}\right): \delta_{\mathrm{C}} 148.2,138.0,137.2,130.8,128.7,128.5,127.4,117.3,64.2$, 
14.4 ppm; HRMS $m / z$ (DART): calcd for $\mathrm{C}_{13} \mathrm{H}_{13} \mathrm{~N}_{2} \mathrm{O}$ (M+H): 229.0972; found: 229.0969; IR (neat): $3151,3127,1768,1471,1385,1297,1285,1220,1158,1004,820,760,700,644 \mathrm{~cm}^{-1}$; m.p.: $56-57^{\circ} \mathrm{C} ; \mathbf{R}_{\mathbf{f}}\left(7: 3\right.$ hexanes/EtOAc; $\left.\mathrm{KMnO}_{4}\right): 0.16$.

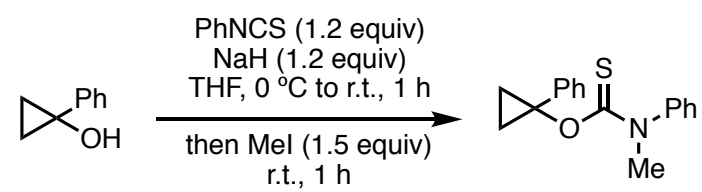

$O$-(1-Phenylcyclopropyl) methyl(phenyl)carbamothioate (1h): To a flame-dried 100-mL flask with a stir bar were added 1-phenylcyclopropanol (1.6 g, $12 \mathrm{mmol}, 1.0$ equiv) and THF ( $40 \mathrm{~mL}$, $0.30 \mathrm{M})$, and the solution was cooled to $0{ }^{\circ} \mathrm{C}$. Phenyl isothiocyanate $(1.5 \mathrm{~mL}, 14 \mathrm{mmol}, 1.2$ equiv) was added, followed by sodium hydride $(0.56 \mathrm{~g}$ of a $60 \% \mathrm{w} / \mathrm{w}$ dispersion in mineral oil, $14 \mathrm{mmol}, 1.2$ equiv) (gas evolves), and the reaction was stirred at r.t. for $1 \mathrm{~h}$. Iodomethane (1.1 $\mathrm{mL}, 18 \mathrm{mmol}, 1.5$ equiv) was added and the reaction was stirred at r.t. for an additional $1 \mathrm{~h}$. The reaction was quenched with sat. aq. $\mathrm{NH}_{4} \mathrm{Cl}$ and extracted with EtOAc $(\times 3)$. The organic fractions were combined, washed with brine $(\times 1)$, dried over $\mathrm{MgSO}_{4}$, and concentrated. The crude residue was purified by flash column chromatography (gradient of $0-10 \%$ EtOAc/hexanes) to yield the product as a white solid (2.4 g, $8.5 \mathrm{mmol}, 71 \%) .{ }^{1} \mathbf{H} \mathbf{~ N M R}\left(400 \mathrm{MHz}, \mathrm{CDCl}_{3}, 298 \mathrm{~K}\right): \delta_{\mathrm{H}} 7.39$ $7.30(\mathrm{~m}, 4 \mathrm{H}), 7.28-7.18(\mathrm{~m}, 3 \mathrm{H}), 7.04-6.98(\mathrm{~m}, 1 \mathrm{H}), 6.74-6.65(\mathrm{~m}, 2 \mathrm{H}), 2.38(\mathrm{~s}, 3 \mathrm{H}), 1.54-1.48$ (m, 2H), 1.33-1.26 (m, 2H) ppm; ${ }^{13} \mathbf{C}$ NMR (100 MHz, CDCl, $\left.298 \mathrm{~K}\right): \delta_{\mathrm{C}} 156.4,147.7,140.4$, 128.9, 128.2, 126.9, 126.1, 123.6, 121.4, 63.4, 16.1, $14.1 \mathrm{ppm}$; HRMS $\mathrm{m} / \mathrm{z}$ (DART): calcd for $\mathrm{C}_{17} \mathrm{H}_{18} \mathrm{NOS}(\mathrm{M}+\mathrm{H}): 284.1104$; found: 284.1107; IR (neat): 3054, 2935, 1634, 1591, 1486, 1151 , 1024, 969, 876, 755, 726, $692 \mathrm{~cm}^{-1}$; m.p.: $53-54^{\circ} \mathrm{C}$; $\mathbf{R}_{\mathbf{f}}$ (5\% EtOAc/hexanes; UV): 0.59 .

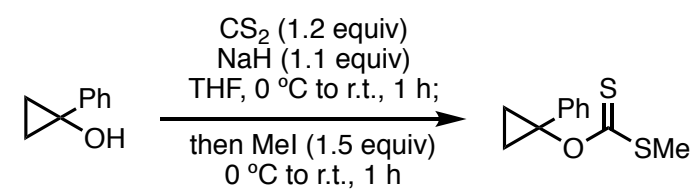

$S$-Methyl $O$-(1-phenylcyclopropyl) carbonodithioate (1i): To a flame-dried 50-mL flask with a stir bar were added 1-phenylcyclopropanol $(0.67 \mathrm{~g}, 5.0 \mathrm{mmol}, 1.0$ equiv) and THF ( $15 \mathrm{~mL}$, $0.33 \mathrm{M})$, and the solution was cooled to $0{ }^{\circ} \mathrm{C} . \mathrm{CS}_{2}(0.36 \mathrm{~mL}, 6.0 \mathrm{mmol}, 1.2$ equiv) was added, followed by sodium hydride $(0.22 \mathrm{~g}$ of a $60 \% \mathrm{w} / \mathrm{w}$ dispersion in mineral oil, $5.5 \mathrm{mmol}, 1.1$ equiv), and the reaction was stirred at r.t. for $1 \mathrm{~h}$. The reaction was cooled to $0{ }^{\circ} \mathrm{C}$ and iodomethane $(0.47 \mathrm{~mL}, 7.5 \mathrm{mmol}, 1.5$ equiv) was added. The reaction was stirred at r.t. for $1 \mathrm{~h}$. The reaction was diluted with $\mathrm{Et}_{2} \mathrm{O}$ and was quenched with sat. aq. $\mathrm{NH}_{4} \mathrm{Cl}$ and extracted with $\mathrm{Et}_{2} \mathrm{O}(\times 2)$. The organic fractions were combined, washed with brine $(\times 1)$, dried over $\mathrm{MgSO}_{4}$, and concentrated. The crude residue was purified by flash column chromatography (gradient of 0 $5 \% \mathrm{EtOAc} /$ hexanes) to yield the product as a yellow oil $(0.93 \mathrm{~g}, 4.1 \mathrm{mmol}, 82 \%) .{ }^{1} \mathbf{H}$ NMR (500 $\left.\mathrm{MHz}, \mathrm{CDCl}_{3}, 298 \mathrm{~K}\right): \delta_{\mathrm{H}} 7.36-7.29(\mathrm{~m}, 2 \mathrm{H}), 7.27-7.20(\mathrm{~m}, 3 \mathrm{H}), 2.53(\mathrm{~s}, 3 \mathrm{H}), 1.58-1.52(\mathrm{~m}, 2 \mathrm{H})$, $1.40-1.35(\mathrm{~m}, 2 \mathrm{H}) \mathrm{ppm} ;{ }^{13} \mathbf{C}$ NMR $\left(125 \mathrm{MHz}, \mathrm{CDCl}_{3}, 298 \mathrm{~K}\right): \delta_{\mathrm{C}} 214.2,139.5,128.4,127.1$, 125.6, 67.4, 19.6, $16.9 \mathrm{ppm}$; HRMS $\mathrm{m} / z$ (DART): calcd for $\mathrm{C}_{11} \mathrm{H}_{13} \mathrm{OS}_{2}(\mathrm{M}+\mathrm{H}): 225.0402$; found: 225.0400; IR (neat): 2921, 1453, 1415, 1224, 1182, 1056, 963, 751, $694 \mathrm{~cm}^{-1} ; \mathbf{R}_{\mathbf{f}}(9: 1$ hexanes/EtOAc; UV/p-anisaldehyde): 0.73 .

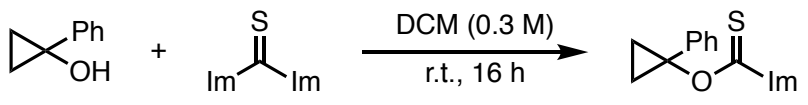

(1 equiv) (1.1 equiv) 
$\boldsymbol{O}$-(1-Phenylcyclopropyl) $\mathbf{1 H}$-imidazole-1-carbothioate (1j): To a flame-dried 25-mL flask with stir bar was added 1-phenylcyclopropanol ( $0.54 \mathrm{~g}, 4.0 \mathrm{mmol}, 1.0$ equiv). The flask was sealed, evacuated and backfilled with $\mathrm{N}_{2}(\times 3)$, and DCM $(13 \mathrm{~mL}, 0.30 \mathrm{M})$ was added. The flask was briefly opened to air and 1,1'-thiocarbonyldiimidazole $(0.71 \mathrm{~g}, 4.4 \mathrm{mmol}, 1.1$ equiv) was added. The reaction was stirred at r.t. under $\mathrm{N}_{2}$ for $16 \mathrm{~h}$. The reaction was opened to air and concentrated. The crude residue was purified by flash column chromatography (gradient of 30 $40 \%$ EtOAc/hexanes) to yield $O$-(1-phenylcyclopropyl) $1 H$-imidazole-1-carbothioate as a yellow solid (0.41 g, $1.7 \mathrm{mmol}, 43 \%)$. ${ }^{1} \mathbf{H}$ NMR (500 MHz, $\left.\mathrm{CDCl}_{3}, 298 \mathrm{~K}\right): \delta_{\mathrm{H}} 8.40-8.32(\mathrm{~m}, 1 \mathrm{H})$, 7.69-7.61 (m, 1H), 7.42-7.30 (m, 4H), 7.30-7.21 (m, 1H), 7.08-7.00 (m, 1H), 1.66-1.58 (m, 2H), 1.52-1.43 (m, 2H) ppm; ${ }^{13} \mathbf{C}$ NMR (125 MHz, $\left.\mathrm{CDCl}_{3}, 298 \mathrm{~K}\right): \delta_{\mathrm{C}} 182.2,137.8,136.5$, 130.7, 128.4, 127.6, 126.1, 117.9, 67.1, $16.0 \mathrm{ppm}$; HRMS $\mathrm{m} / z$ (DART): calcd for $\mathrm{C}_{13} \mathrm{H}_{13} \mathrm{~N}_{2} \mathrm{OS}$ $(\mathrm{M}+\mathrm{H}): 245.0743$; found: 245.0745; IR (neat): 3151, 3127, 1528, 1475, 1459, 1383, 1332, 1280 , 1231, 1204, 1110, 1043, 979, 951, 818, 697, $651 \mathrm{~cm}^{-1}$; m.p.: $50-52{ }^{\circ} \mathrm{C} ; \mathbf{R}_{\mathbf{f}}(7: 3$ hexanes/EtOAc; $\left.\mathrm{KMnO}_{4}\right): 0.21$. 


\section{E.2. Preparation of standard thiocarbamate substrates}

General Procedure D: Preparation of thiocarbamate starting materials

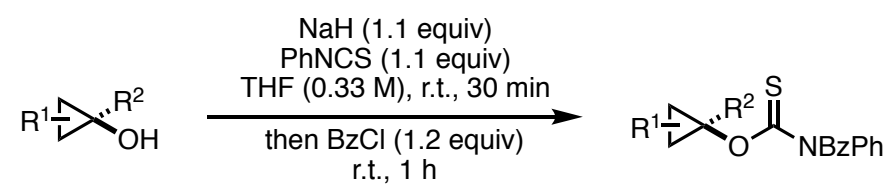

To a flame-dried 50-mL flask with stir bar was added cyclopropanol (1.0 equiv). The flask was evacuated and backfilled with $\mathrm{N}_{2}$ and THF $(0.33 \mathrm{M})$ was added. Phenyl isothiocyanate (1.1 equiv) was added, followed by sodium hydride ( $60 \%$ dispersion in mineral oil, 1.1 equiv), and the reaction was stirred at r.t. for $0.50-16 \mathrm{~h}$. Then, benzoyl chloride (1.2 equiv) was added, and the reaction was stirred at r.t. for $1-16 \mathrm{~h}$. The reaction was opened to air, quenched with sat. aq. $\mathrm{NH}_{4} \mathrm{Cl}$, and extracted with EtOAc $(\times 3)$. The organic fractions were combined, washed with brine $(\times 1)$, dried over $\mathrm{MgSO}_{4}$, and concentrated. The crude residue was purified by flash column chromatography and the fractions containing desired product were collected, combined, and concentrated. If the concentrate solidified, it was recrystallized (DCM/hexanes) or washed (hexanes or $\mathrm{Et}_{2} \mathrm{O}$ ) to yield the desired product. If the concentrate was a foam, it was stripped of solvent (hexanes or $\mathrm{Et}_{2} \mathrm{O}$ ) and/or triturated (hexanes) to yield the desired product.<smiles>S=C(OC1CC1)N(c1ccccc1)c1ccccc1</smiles>

$O$-(1-Phenylcyclopropyl) benzoyl(phenyl)carbamothioate (1k): Prepared according to General Procedure D by stirring for 30 min after the addition of NaH and PhNCS and for $1 \mathrm{~h}$ after the addition of $\mathrm{BzCl}$. The crude material was triturated (hexanes), then recrystallized (DCM/pentane, 3 crops) to yield the product as a pale yellow solid (Figure S5). The product was prepared on $21-\mathrm{mmol}$ scale (3.5 g isolated, $9.4 \mathrm{mmol}, 45 \%)$ and on $4.0-\mathrm{mmol}$ scale $(0.97 \mathrm{~g}$, isolated, $2.6 \mathrm{mmol}, 65 \%) .{ }^{1} \mathbf{H}$ NMR $\left(500 \mathrm{MHz}, \mathrm{CDCl}_{3}, 298 \mathrm{~K}\right): \delta_{\mathrm{H}} 7.86-7.82(\mathrm{~m}, 2 \mathrm{H}), 7.61-7.57$ $(\mathrm{m}, 1 \mathrm{H}), 7.59-7.45(\mathrm{~m}, 2 \mathrm{H}), 7.44-7.39(\mathrm{~m}, 2 \mathrm{H}), 7.37-7.33(\mathrm{~m}, 1 \mathrm{H}), 7.32-7.36(\mathrm{~m}, 4 \mathrm{H}), 7.24-$ $7.20(\mathrm{~m}, 1 \mathrm{H}), 7.20-7.16(\mathrm{~m}, 2 \mathrm{H}), 1.19-1.08(\mathrm{~m}, 2 \mathrm{H}), 0.97-0.86(\mathrm{~m}, 2 \mathrm{H}) \mathrm{ppm} ;{ }^{13} \mathbf{C}$ NMR $(125$ $\left.\mathrm{MHz}, \mathrm{CDCl}_{3}, 298 \mathrm{~K}\right): \delta_{\mathrm{C}} 190.8,172.3,141.7,139.1,136.0,132.9,129.6,128.9,128.8,128.4$, 128.4, 128.3, 127.2, 126.2, 66.0, 16.3 ppm; HRMS m/z (DART): calcd for $\mathrm{C}_{23} \mathrm{H}_{20} \mathrm{NO}_{2} \mathrm{~S}(\mathrm{M}+\mathrm{H}$ ): 374.1209; found: 374.1203; IR (neat): 3053, 3009, 1686, 1591, 1489, 1449, 1294, 1271, 1232, 1176, 1070, 1028, 999, 755, $690 \mathrm{~cm}^{-1}$; m.p.: $97-99^{\circ} \mathrm{C} ; \mathbf{R}_{\mathbf{f}}$ (5\% EtOAc/hexanes; UV): 0.31. 


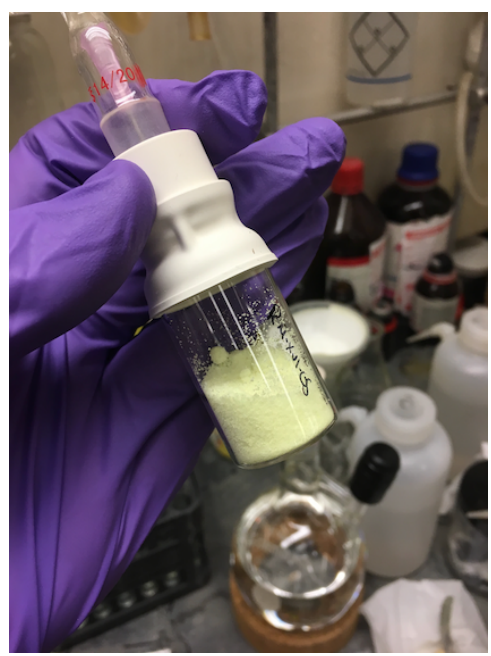

Figure S5. Appearance of thiocarbamate substrate $\mathbf{1 k}$.<smiles>S=C(Nc1ccccc1)OC1(c2ccco2)CC1</smiles>

O-(1-(Furan-2-yl)cyclopropyl) benzoyl(phenyl)carbamothioate (S7): Prepared on 2.0-mmol scale by stirring for 30 min after the addition of $\mathrm{NaH}$ and $\mathrm{PhNCS}$ and for $16 \mathrm{~h}$ after the addition of $\mathrm{BzCl}$. The crude material was purified by flash column chromatography (gradient of 0-20\% EtOAc/hexanes), then washed with hexanes to yield the product as a pale yellow solid ( $0.29 \mathrm{~g}$, $0.80 \mathrm{mmol}, 40 \%) .{ }^{1} \mathbf{H}$ NMR $\left(500 \mathrm{MHz}, \mathrm{CDCl}_{3}, 298 \mathrm{~K}\right): \delta_{\mathrm{H}} 7.83-7.78(\mathrm{~m}, 2 \mathrm{H}), 7.60-7.55(\mathrm{~m}$, $1 \mathrm{H}), 7.47-7.39(\mathrm{~m}, 4 \mathrm{H}), 7.37-7.33(\mathrm{~m}, 1 \mathrm{H}), 7.31-7.26(\mathrm{~m}, 3 \mathrm{H}), 6.35(\mathrm{dd}, J=3.3,0.9 \mathrm{~Hz}, 1 \mathrm{H})$, $6.30(\mathrm{dd}, J=3.3,1.8 \mathrm{~Hz}, 1 \mathrm{H}), 1.18-1.13(\mathrm{~m}, 2 \mathrm{H}), 0.91-0.86(\mathrm{~m}, 2 \mathrm{H}) \mathrm{ppm} ;{ }^{13} \mathbf{C} \mathbf{N M R}(125 \mathrm{MHz}$, $\left.\mathrm{CDCl}_{3}, 298 \mathrm{~K}\right): \delta_{\mathrm{C}} 190.6,172.1,151.8,141.8,141.7,135.9,132.8,129.6,128.8,128.7,128.4$, 128.3, 110.5, 109.5, 59.9, $14.7 \mathrm{ppm}$; HRMS $m / z$ (DART): calcd for $\mathrm{C}_{21} \mathrm{H}_{18} \mathrm{NO}_{3} \mathrm{~S}(\mathrm{M}+\mathrm{H})$ : 364.1002; found: 364.1001; IR (neat): 1686, 1595, 1489, 1448, 1295, 1271, 1244, 1186, 1167 , 1006, 732, 698, $690 \mathrm{~cm}^{-1}$; m.p.: $71-72{ }^{\circ} \mathrm{C} ; \mathbf{R}_{\mathbf{f}}(9: 1$ hexanes/EtOAc; UV): 0.29.<smiles>S=C(Nc1ccccc1)OC1(c2cccs2)CC1</smiles>

$O$-(1-(Thiophen-2-yl)cyclopropyl) benzoyl(phenyl)carbamothioate (S8): Prepared on 3.1mmol scale scale by stirring for 30 min after the addition of $\mathrm{NaH}$ and $\mathrm{PhNCS}$ and for $16 \mathrm{~h}$ after the addition of $\mathrm{BzCl}$. The crude material was purified by flash column chromatography (gradient of $0-20 \%$ EtOAc/hexanes), then triturated with hexanes to yield the product as a pale yellow solid (0.79 g, $2.1 \mathrm{mmol}, 68 \%)$. ${ }^{1} \mathbf{H}$ NMR $\left(500 \mathrm{MHz}, \mathrm{CDCl}_{3}, 298 \mathrm{~K}\right): \delta_{\mathrm{H}} 7.84-7.80(\mathrm{~m}, 2 \mathrm{H}), 7.59$ $7.53(\mathrm{~m}, 1 \mathrm{H}), 7.47-7.38(\mathrm{~m}, 4 \mathrm{H}), 7.38-7.32(\mathrm{~m}, 1 \mathrm{H}), 7.31-7.26(\mathrm{~m}, 2 \mathrm{H}), 7.21(\mathrm{dd}, J=5.2,1.4$ $\mathrm{Hz}, 1 \mathrm{H}), 6.97(\mathrm{dd}, J=3.6,1.4 \mathrm{~Hz}, 1 \mathrm{H}), 6.89(\mathrm{dd}, J=5.2,3.7 \mathrm{~Hz}, 1 \mathrm{H}), 1.21-1.14(\mathrm{~m}, 2 \mathrm{H}), 1.03-$ 0.98 (m, 2H) ppm; ${ }^{13} \mathbf{C}$ NMR (125 MHz, $\left.\mathrm{CDCl}_{3}, 298 \mathrm{~K}\right): \delta_{\mathrm{C}} 190.5,172.1,142.6,141.7,135.8$, $132.8,129.6,128.8,128.7,128.4,128.3,127.2,126.3,125.8,61.9,16.7$ ppm; HRMS m/z (DART): calcd for $\mathrm{C}_{21} \mathrm{H}_{18} \mathrm{NO}_{2} \mathrm{~S}_{2}(\mathrm{M}+\mathrm{H}): 380.0774$; found: 380.0775 ; IR (neat): 3052, 1682, 1491, 1448, 1293, 1270, 1171, 1006, 697, $690 \mathrm{~cm}^{-1}$; m.p.: $80-82^{\circ} \mathrm{C}$; $\mathbf{R}_{\mathbf{f}}(9: 1$ hexanes/EtOAc; UV): 0.19 . 


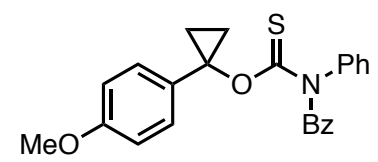

O-(1-(4-methoxyphenyl)cyclopropyl) benzoyl(phenyl)carbamothioate (S9): Prepared on 6.1mmol scale by stirring for $30 \mathrm{~min}$ after the addition of $\mathrm{NaH}$ and PhNCS and for $1 \mathrm{~h}$ after the addition of $\mathrm{BzCl}$. The crude material was purified by flash column chromatography (gradient of 0-30\% EtOAc/hexanes), then washed with hexanes to yield the product as a white solid (1.8 g, $4.8 \mathrm{mmol}, 79 \%) .{ }^{1} \mathbf{H}$ NMR $\left(500 \mathrm{MHz}, \mathrm{CDCl}_{3}, 298 \mathrm{~K}\right): \delta_{\mathrm{H}} 7.84-7.79(\mathrm{~m}, 2 \mathrm{H}), 7.61-7.55(\mathrm{~m}, 1 \mathrm{H})$, 7.49-7.43 (m, 2H), 7.43-7.37 (m, 2H), 7.37-7.31 (m, 1H), 7.29-7.24 (m, 2H), 7.21-7.16 (m, 2H), 6.83-6.77 (m, 2H), 3.79 (s, 3H), 1.10-1.00 (m, 2H), 0.94-0.84 (m, 2H) ppm; ${ }^{13} \mathbf{C ~ N M R}$ $\left(125 \mathrm{MHz}, \mathrm{CDCl}_{3}, 298 \mathrm{~K}\right): \delta_{\mathrm{C}} 190.8,172.3,159.0,141.7,136.1,132.8,130.8,129.6,129.1$, 128.9, 128.7, 128.4, 128.3, 113.5, 66.1, 55.4, $15.1 \mathrm{ppm}$; HRMS $m / z$ (DART): calcd for $\mathrm{C}_{24} \mathrm{H}_{22} \mathrm{NO}_{3} \mathrm{~S}(\mathrm{M}+\mathrm{H}): 404.1315$; found: 404.1316; IR (neat): 3007, 2835, 1689, 1613, 1516 , 1448, 1316, 1276, 1251, 1192, 1170, 1024, 825, $695 \mathrm{~cm}^{-1}$; m.p.: 77-80 ${ }^{\circ} \mathrm{C} ; \mathbf{R}_{\mathbf{f}}(9: 1$ hexanes/EtOAc; UV): 0.23.<smiles>FC(F)(F)c1ccc(C2(OC(=S)N(c3ccccc3)c3ccccc3)CC2)cc1</smiles>

$O$-(1-(4-(Trifluoromethyl)phenyl)cyclopropyl) benzoyl(phenyl)carbamothioate (S10): Prepared on 1.0-mmol scale by stirring for $30 \mathrm{~min}$ after the addition of $\mathrm{NaH}$ and $\mathrm{PhNCS}$ and for $16 \mathrm{~h}$ after the addition of $\mathrm{BzCl}$. The crude material was purified by flash column chromatography (gradient of 0-20\% EtOAc/hexanes), then washed with hexanes to yield the product as a white solid (0.26 g, $0.59 \mathrm{mmol}, 59 \%)$. ${ }^{1} \mathbf{H}$ NMR (500 MHz, $\left.\mathrm{CDCl}_{3}, 298 \mathrm{~K}\right): \delta_{\mathrm{H}} 7.87-7.81(\mathrm{~m}, 2 \mathrm{H})$, 7.64-7.57 (m, 1H), 7.57-7.51 (m, 2H), 7.51-7.45 (m, 2H), 7.45-7.40 (m, 2H), 7.38-7.33 (m, 1H), 7.33-7.29 (m, 2H), 7.28-7.23 (m, 2H), 1.23-1.15 (m, 2H), 1.04-0.97 (m, 2H) ppm; ${ }^{19} \mathbf{F}$ NMR $\left(376 \mathrm{MHz}, \mathrm{CDCl}_{3}, 298 \mathrm{~K}\right): \delta_{\mathrm{F}}-62.4 \mathrm{ppm} ;{ }^{13} \mathbf{C} \mathbf{N M R}\left(125 \mathrm{MHz}, \mathrm{CDCl}_{3}, 298 \mathrm{~K}\right): \delta_{\mathrm{C}} 190.6$, $172.1,143.5$ (q, $J=1.3 \mathrm{~Hz}), 141.6,135.8,133.1,129.7,129.2(\mathrm{q}, J=32.4 \mathrm{~Hz}), 129.0,128.9$, 128.6, 128.4, 126.0, 125.4 (q, $J=3.8 \mathrm{~Hz}$ ), 65.3, $17.1 \mathrm{ppm}$; HRMS $m / z$ (DART): calcd for $\mathrm{C}_{24} \mathrm{H}_{19} \mathrm{NO}_{2} \mathrm{~F}_{3} \mathrm{~S}(\mathrm{M}+\mathrm{H}): 442.1083$; found: 442.1082; IR (neat): 3180, 3034, 1689, 1683, 1600, 1553, 1451, 1323, 1278, 1166, 1115, 1098, 1067, 1011, $830 \mathrm{~cm}^{-1}$; m.p.: $78-80{ }^{\circ} \mathrm{C} ; \mathbf{R}_{\mathbf{f}}(9: 1$ hexanes/EtOAc; UV): 0.50 .<smiles>Fc1ccc(C2(OC(=S)Nc3ccccc3)CC2)cc1</smiles>

O-(1-(4-Fluorophenyl)cyclopropyl) benzoyl(phenyl)carbamothioate (S11): Prepared on 3.9mmol scale by stirring for 30 min after the addition of $\mathrm{NaH}$ and $\mathrm{PhNCS}$ and for $16 \mathrm{~h}$ after the addition of $\mathrm{BzCl}$. The crude material was purified by flash column chromatography (gradient of 0-20\% EtOAc/hexanes), then triturated with hexanes to yield the product as an off-white solid (0.60 g, $1.5 \mathrm{mmol}, 38 \%)$. ${ }^{1} \mathbf{H}$ NMR (400 MHz, $\left.\mathrm{CDCl}_{3}, 298 \mathrm{~K}\right): \delta_{\mathrm{H}} 7.90-7.81(\mathrm{~m}, 2 \mathrm{H}), 7.65-7.59$ (m, 1H), 7.54-7.19 (m, 9H), 7.04-6.95 (m, 2H), 1.16-1.08 (m, 2H), 1.02-0.94 (m, 2H) ppm; ${ }^{19} \mathbf{F}$ NMR (376 MHz, $\left.\mathrm{CDCl}_{3}, 298 \mathrm{~K}\right): \delta_{\mathrm{F}}-114.7 \mathrm{ppm} ;{ }^{13} \mathbf{C} \mathbf{N M R}\left(100 \mathrm{MHz}, \mathrm{CDCl}_{3}, 298 \mathrm{~K}\right): \delta_{\mathrm{C}}$ 190.7, 172.2, $162.0(\mathrm{~d}, J=244.7), 141.6,135.9,132.9,129.6,129.2,129.1,128.9,128.8,128.4$, 128.4, 115.1 (d, $J=21.5 \mathrm{~Hz}), 65.7,15.6 \mathrm{ppm}$; HRMS $\mathrm{m} / z$ (DART): calcd for $\mathrm{C}_{23} \mathrm{H}_{19} \mathrm{NO}_{2} \mathrm{FS}$ 
$(\mathrm{M}+\mathrm{H})$ : 392.1115; found: 392.1111; IR (neat): 3199, 3032, 1683, 1596, 1512, 1449, 1315, 1271, 1189, 1173, 1022, 832, $694 \mathrm{~cm}^{-1}$; m.p.: 101-103 ${ }^{\circ} \mathrm{C} ; \mathbf{R}_{\mathbf{f}}(9: 1$ hexanes/EtOAc; UV): 0.52 .<smiles>S=C(Nc1ccccc1)OC1(c2ccc(Cl)cc2)CC1</smiles>

$O$-(1-(4-Chlorophenyl)cyclopropyl) benzoyl(phenyl)carbamothioate (S12): Prepared on 2.0mmol scale by stirring for 30 min after the addition of $\mathrm{NaH}$ and PhNCS and for $16 \mathrm{~h}$ after the addition of $\mathrm{BzCl}$. The crude material was purified by flash column chromatography (gradient of 0-20\% EtOAc/hexanes), then washed with hexanes to yield the product as a white solid $(0.51 \mathrm{~g}$, $1.3 \mathrm{mmol}, 65 \%) .{ }^{1} \mathbf{H}$ NMR $\left(500 \mathrm{MHz}, \mathrm{CDCl}_{3}, 298 \mathrm{~K}\right): \delta_{\mathrm{H}} 7.85-7.79(\mathrm{~m}, 2 \mathrm{H}), 7.62-7.57(\mathrm{~m}, 1 \mathrm{H})$, 7.49-7.44 (m, 2H), 7.44-7.39 (m, 2H), 7.37-7.32 (m, 1H), 7.30-7.22 (m, 4H), 7.15-7.10 (m, 2H), 1.15-1.07 (m, 2H), 0.98-0.91 (m, 2H) ppm; $\left.{ }^{13} \mathbf{C ~ N M R ~ ( 1 2 6 ~ M H z , ~} \mathrm{CDCl}_{3}, 298 \mathrm{~K}\right): \delta_{\mathrm{C}}$ 190.7, 172.2, 141.6, 137.6, 135.9, 133.1, 133.0, 129.6, 128.9, 128.8, 128.5, 128.4, 128.4, 128.1, 65.5, 16.1; HRMS $m / z$ (DART): calcd for $\mathrm{C}_{23} \mathrm{H}_{19} \mathrm{NO}_{2} \mathrm{SCl}(\mathrm{M}+\mathrm{H})$ : 408.0820; found: 408.0813; IR (neat): 1686, 1595, 1494, 1450, 1317, 1296, 1269, 1232, 1191, 1171, 1095, 1014, 825, 694 $\mathrm{cm}^{-1}$; m.p.: $111-112^{\circ} \mathrm{C} ; \mathbf{R}_{\mathbf{f}}(9: 1$ hexanes/EtOAc; UV): 0.41 .<smiles>S=C(OC1(c2ccc(-c3ccccc3)cc2)CC1)N(Cc1ccccc1)c1ccccc1</smiles>

$O$-(1-([1,1'-biphenyl]-4-yl)cyclopropyl) benzoyl(phenyl)carbamothioate (S13): Prepared on 3.0-mmol scale by stirring for $30 \mathrm{~min}$ after the addition of $\mathrm{NaH}$ and $\mathrm{PhNCS}$ and for $16 \mathrm{~h}$ after the addition of $\mathrm{BzCl}$. The crude material was purified by flash column chromatography (gradient of 0-20\% EtOAc/hexanes), then triturated with hexanes to yield the product as a tan solid ( $0.58 \mathrm{~g}$, $1.3 \mathrm{mmol}, 43 \%) .{ }^{1} \mathbf{H}$ NMR $\left(500 \mathrm{MHz}, \mathrm{CDCl}_{3}, 298 \mathrm{~K}\right): \delta_{\mathrm{H}} 7.88-7.83(\mathrm{~m}, 2 \mathrm{H}), 7.62-7.54(\mathrm{~m}, 4 \mathrm{H})$, 7.53-7.46 (m, 3H), 7.45-7.40 (m, 4H), 7.39-7.30 (m, 4H), 7.26-7.23 (m, 2H), 1.20-1.15 (m, 2H), 0.98-0.93 (m, 2H) ppm; ${ }^{13} \mathbf{C}$ NMR (125 MHz, $\left.\mathrm{CDCl}_{3}, 298 \mathrm{~K}\right): \delta_{\mathrm{C}} 190.7,172.1,141.6$, 140.6, 140.0, 138.0, 135.9, 132.8, 129.5, 128.8, 128.7, 128.7, 128.6, 128.3, 127.3, 127.0, 126.9, 126.5, 65.7, $16.2 \mathrm{ppm}$; HRMS $m / z$ (DART): calcd for $\mathrm{C}_{29} \mathrm{H}_{24} \mathrm{NO}_{2} \mathrm{~S}(\mathrm{M}+\mathrm{H})$ : 450.1522; found: 450.1525; IR (neat): 3031, 1699, 1547, 1488, 1279, 1175, 1064, 831, 764, $699 \mathrm{~cm}^{-1}$; m.p.: 88$90{ }^{\circ} \mathrm{C} ; \mathbf{R}_{\mathbf{f}}(9: 1$ hexanes/EtOAc; UV): 0.53.

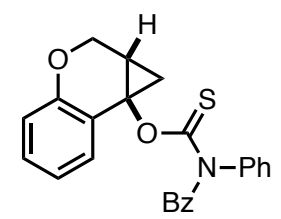

$O$-((trans)-1a,2-Dihydrocyclopropa[c]chromen-7b(1H)-yl) benzoyl(phenyl)carbamothioate (S14): Prepared on 3.0-mmol scale by stirring for $30 \mathrm{~min}$ after the addition of $\mathrm{NaH}$ and $\mathrm{PhNCS}$ and for $16 \mathrm{~h}$ after the addition of $\mathrm{BzCl}$. The crude material was purified by flash column chromatography (gradient of 0-20\% EtOAc/hexanes), then stripped with $\mathrm{Et}_{2} \mathrm{O} /$ hexanes and washed with hexanes to yield the product as a white solid $(0.56 \mathrm{~g}, 1.4 \mathrm{mmol}, 47 \%) .{ }^{\mathbf{1}} \mathbf{H}$ NMR $\left(500 \mathrm{MHz}, \mathrm{CDCl}_{3}, 298 \mathrm{~K}\right): \delta_{\mathrm{H}} 7.92-7.86(\mathrm{~m}, 2 \mathrm{H}), 7.63-7.58(\mathrm{~m}, 1 \mathrm{H}), 7.53-7.42(\mathrm{~m}, 4 \mathrm{H}), 7.40$ $7.35(\mathrm{~m}, 3 \mathrm{H}), 7.30(\mathrm{dd}, J=7.7,1.8 \mathrm{~Hz}, 1 \mathrm{H}), 7.10(\mathrm{ddd}, J=8.1,7.5,1.7 \mathrm{~Hz}, 1 \mathrm{H}), 6.97(\mathrm{td}, J=$ 7.6, 1.2 Hz, 1H), $6.81(\mathrm{dd}, J=8.1,1.2 \mathrm{~Hz}, 1 \mathrm{H}), 4.18(\mathrm{dd}, J=10.5,1.5 \mathrm{~Hz}, 1 \mathrm{H}), 4.11-4.07$ (m, 
$1 \mathrm{H}), 1.89$ (ddt, $J=10.1,7.0,1.6 \mathrm{~Hz}, 1 \mathrm{H}), 1.16(\mathrm{tdd}, J=7.0,0.9,0.7 \mathrm{~Hz}, 1 \mathrm{H}), 0.74(\mathrm{dd}, J=10.3$, $6.1 \mathrm{~Hz}, 1 \mathrm{H}) \mathrm{ppm} ;{ }^{13} \mathbf{C} \mathbf{N M R}\left(125 \mathrm{MHz}, \mathrm{CDCl}_{3}, 298 \mathrm{~K}\right): \delta_{\mathrm{C}} 190.9,172.1,150.6,141.5,135.8$, 133.1, 129.7, 129.0, 128.9, 128.6, 128.3, 127.4, 125.5, 124.3, 121.7, 117.5, 61.7, 60.5, 27.8, 17.6 ppm; HRMS m/z (DART): calcd for $\mathrm{C}_{24} \mathrm{H}_{20} \mathrm{NO}_{3} \mathrm{~S}(\mathrm{M}+\mathrm{H}): 402.1158$; found: 402.1162 ; IR (neat): 3056, 3034, 1692, 1584, 1490, 1460, 1263, 1186, 1177, 1066, 1034, 750, $697 \mathrm{~cm}^{-1}$; m.p.: $118-$ $119^{\circ} \mathrm{C} ; \mathbf{R}_{\mathbf{f}}(9: 1$ hexanes/EtOAc; UV/KMnO 4$): 0.39$.

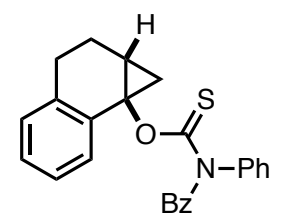

\section{O-((trans)-1,1a,2,3-Tetrahydro-7bH-cyclopropa[a]naphthalen-7b-yl)}

benzoyl(phenyl)carbamothioate (S15): Prepared on 4.0-mmol scale by stirring for 30 min after the addition of $\mathrm{NaH}$ and $\mathrm{PhNCS}$ and for $16 \mathrm{~h}$ after the addition of $\mathrm{BzCl}$. The crude material was purified by flash column chromatography (gradient of 0-20\% EtOAc/hexanes), then stripped with $\mathrm{Et}_{2} \mathrm{O} /$ hexanes and washed with hexanes to yield the product as a white solid $(1.1 \mathrm{~g}, 2.8$ mmol, 70\%). ${ }^{1} \mathbf{H}$ NMR (500 MHz, $\left.\mathrm{CDCl}_{3}, 298 \mathrm{~K}\right): \delta_{\mathrm{H}} 7.91-7.86(\mathrm{~m}, 2 \mathrm{H}), 7.62-7.57$ (m, 1H), 7.57-7.41 (m, 4H), 7.39-7.32 (m, 4H), $7.21(\mathrm{tt}, J=7.5,1.1 \mathrm{~Hz}, 1 \mathrm{H}), 7.10$ (td, $J=7.5,1.4 \mathrm{~Hz}$, $1 \mathrm{H}), 7.03(\mathrm{dt}, J=7.5,1.3 \mathrm{~Hz}, 1 \mathrm{H}), 2.68-2.61(\mathrm{~m}, 1 \mathrm{H}), 2.35-2.25(\mathrm{~m}, 1 \mathrm{H}), 2.02-1.90(\mathrm{~m}, 2 \mathrm{H})$, $1.72-1.65(\mathrm{~m}, 1 \mathrm{H}), 0.94(\mathrm{t}, J=6.8 \mathrm{~Hz}, 1 \mathrm{H}), 0.50(\mathrm{dd}, J=10.3,6.3 \mathrm{~Hz}, 1 \mathrm{H}) \mathrm{ppm} ;{ }^{13} \mathbf{C}$ NMR $(126$ $\left.\mathrm{MHz}, \mathrm{CDCl}_{3}, 298 \mathrm{~K}\right): \delta_{\mathrm{C}} 191.2,172.3,141.8,136.2,136.1,132.9,132.1,129.7,128.9,128.8$, 128.8, 128.4, 128.4, 126.2, 126.0, 124.0, 64.0, 25.8, 24.8, 18.1, 16.0 ppm; HRMS m/z (DART): calcd for $\mathrm{C}_{25} \mathrm{H}_{22} \mathrm{NO}_{2} \mathrm{~S}(\mathrm{M}+\mathrm{H})$ : 400.1366; found: 400.1374; IR (neat): 3037, 2932, 2915, 1694, 1596, 1491, 1449, 1312, 1269, 1192, 1174, 1060, 854, 751, 719, $695 \mathrm{~cm}^{-1}$; m.p.: $110-112{ }^{\circ} \mathrm{C} ; \mathbf{R}_{\mathbf{f}}$ (9:1 hexanes/EtOAc; UV): 0.32.<smiles>O=C(c1ccccc1)N(C(=S)OC12CC(Cc3ccccc31)C2)c1ccccc1</smiles>

O-((trans)-6,6a-dihydrocyclopropa[a]inden-1a(1H)-yl) benzoyl(phenyl)carbamothioate (S16): Prepared on 3.0-mmol scale by stirring for 30 min after the addition of $\mathrm{NaH}$ and $\mathrm{PhNCS}$ and for $16 \mathrm{~h}$ after the addition of $\mathrm{BzCl}$. The crude material was purified by flash column chromatography (gradient of 0-20\% EtOAc/hexanes), then stripped with hexanes and washed with $\mathrm{Et}_{2} \mathrm{O}$ to yield the product as an off-white solid (0.72 $\left.\mathrm{g}, 1.9 \mathrm{mmol}, 62 \%\right)$. ${ }^{1} \mathbf{H}$ NMR (500 $\left.\mathrm{MHz}, \mathrm{CDCl}_{3}, 298 \mathrm{~K}\right): \delta_{\mathrm{H}} 7.89-7.84(\mathrm{~m}, 2 \mathrm{H}), 7.62-7.58(\mathrm{~m}, 1 \mathrm{H}), 7.51-7.42(\mathrm{~m}, 4 \mathrm{H}), 7.40-7.33$ $(\mathrm{m}, 4 \mathrm{H}), 7.19-7.12(\mathrm{~m}, 2 \mathrm{H}), 7.11-7.07(\mathrm{~m}, 1 \mathrm{H}), 3.27-3.21(\mathrm{~m}, 1 \mathrm{H}), 2.67(\mathrm{~d}, J=17.1 \mathrm{~Hz}, 1 \mathrm{H})$, $1.79-1.74(\mathrm{~m}, 1 \mathrm{H}), 1.01(\mathrm{ddd}, J=9.7,5.8,0.5 \mathrm{~Hz}, 1 \mathrm{H}), 0.55(\mathrm{dd}, J=5.6,5.6 \mathrm{~Hz}, 1 \mathrm{H}) \mathrm{ppm} ;{ }^{13} \mathbf{C}$ NMR $\left(125 \mathrm{MHz}, \mathrm{CDCl}_{3}, 298 \mathrm{~K}\right): \delta_{\mathrm{C}} 191.6,172.2,142.5,141.8,139.8,136.0,132.9,129.7$, $128.9,128.8,128.4,128.3,127.3,126.3,125.7,122.7,73.9,34.3,24.2,22.4$ ppm; HRMS $\mathrm{m} / z$ (DART): calcd for $\mathrm{C}_{24} \mathrm{H}_{20} \mathrm{NO}_{2} \mathrm{~S}(\mathrm{M}+\mathrm{H}): 386.1209$; found: 386.1214 ; IR (neat): 3043, 1693 , $1598,1494,1450,1319,1270,1194,1175,1065,854,694 \mathrm{~cm}^{-1}$; m.p.: $117-119^{\circ} \mathrm{C} ; \mathbf{R}_{\mathbf{f}}(9: 1$ hexanes/EtOAc; UV): 0.45. 


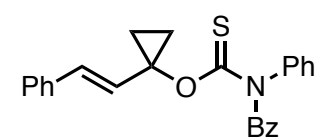

(E)-O-(1-Styrylcyclopropyl) benzoyl(phenyl)carbamothioate (S17): Prepared on 2.2-mmol scale by stirring for 30 min after the addition of $\mathrm{NaH}$ and PhNCS and for $16 \mathrm{~h}$ after the addition of $\mathrm{BzCl}$. The crude material was purified by flash column chromatography (gradient of 0-20\% EtOAc/hexanes), then triturated with hexanes to yield the product as an off-white solid $(0.15 \mathrm{~g}$, $0.38 \mathrm{mmol}, 17 \%) .{ }^{1} \mathbf{H}$ NMR $\left(500 \mathrm{MHz}, \mathrm{CDCl}_{3}, 298 \mathrm{~K}\right): \delta_{\mathrm{H}} 7.87-7.82(\mathrm{~m}, 2 \mathrm{H}), 7.61-7.56(\mathrm{~m}$, 1H), 7.50-7.42 (m, 4H), 7.40-7.27 (m, 7H), 7.25-7.20 (m, 1H), $6.34(\mathrm{~d}, J=16.1 \mathrm{~Hz}, 1 \mathrm{H}), 6.25$ $(\mathrm{d}, J=16.1 \mathrm{~Hz}, 1 \mathrm{H}), 1.04-0.94(\mathrm{~m}, 2 \mathrm{H}), 0.84-0.74(\mathrm{~m}, 2 \mathrm{H}) \mathrm{ppm} ;{ }^{13} \mathbf{C} \mathbf{N M R}\left(125 \mathrm{MHz}, \mathrm{CDCl}_{3}\right.$, $298 \mathrm{~K}): \delta_{\mathrm{C}} 191.0,172.2,141.8,136.5,136.1,132.9,129.7,129.4,128.9,128.7,128.7,128.5$, 128.4, 127.8, 127.7, 126.5, 65.1, 15.9 ppm; HRMS $m / z$ (DART): calcd for $\mathrm{C}_{25} \mathrm{H}_{22} \mathrm{NO}_{2} \mathrm{~S}(\mathrm{M}+\mathrm{H})$ : 400.1366; found: 400.1367; IR (neat): 3028, 1691, 1597, 1495, 1448, 1298, 1283, 1258, 1191 , 1174, 1062, 853, 742, $692 \mathrm{~cm}^{-1}$; m.p.: $145-147^{\circ} \mathrm{C}$; $\mathbf{R}_{\mathbf{f}}(9: 1$ hexanes/EtOAc; UV/KMnO $): 0.41$.<smiles>[18OH]N(C(=S)OC1CC1)c1ccccc1</smiles>

$O$-(1-benzylcyclopropyl) benzoyl(phenyl)carbamothioate (S18): Prepared according to General Procedure D on 4.0-mmol scale by stirring for $30 \mathrm{~min}$ after the addition of $\mathrm{NaH}$ and $\mathrm{PhNCS}$ and for $1 \mathrm{~h}$ after the addition of $\mathrm{BzCl}$. The crude residue was purified by flash column chromatography (gradient of $0-10 \%$ EtOAc/hexanes), then recrystallized (DCM/hexanes) to yield the product as a white solid (1.2 g, $3.1 \mathrm{mmol}, 78 \%) .{ }^{\mathbf{1}} \mathbf{H} \mathbf{N M R}\left(400 \mathrm{MHz}, \mathrm{CDCl}_{3}, 298 \mathrm{~K}\right)$ : $\delta_{\mathrm{H}} 7.74-7.85(\mathrm{~m}, 2 \mathrm{H}), 7.62-7.54(\mathrm{~m}, 1 \mathrm{H}), 7.51-7.16(\mathrm{~m}, 12 \mathrm{H}), 3.38(\mathrm{~s}, 2 \mathrm{H}), 0.69-0.49(\mathrm{~m}, 4 \mathrm{H})$ ppm; ${ }^{13} \mathbf{C}$ NMR $\left(100 \mathrm{MHz}, \mathrm{CDCl}_{3}, 298 \mathrm{~K}\right): \delta_{\mathrm{C}} 191.1,172.0,141.4,137.3,135.8,132.8,129.7$, 129.6, 128.8, 128.7, 128.4, 128.3, 128.3, 126.8, 66.1, 38.9, 12.0 ppm; HRMS m/z (DART): calcd for $\mathrm{C}_{24} \mathrm{H}_{22} \mathrm{NO}_{2} \mathrm{~S}(\mathrm{M}+\mathrm{H})$ : 388.1366; found: 388.1375; IR (neat); 3020, 1695, 1490, 1449, 1300, 1270, 1244, 1191, 1173, 1154, 1064, 980, 856, 695, $688 \mathrm{~cm}^{-1}$; m.p.: $76-78^{\circ} \mathrm{C} ; \mathbf{R}_{\mathbf{f}}(5 \%$ EtOAc/hexanes; $\mathrm{UV} / \mathrm{KMnO}_{4}$ ): 0.33 .<smiles>CC1=C(/C=C/C2(OC(=S)N(Cc3ccccc3)c3ccccc3)CC2)C(C)(C)CCC1</smiles>

\section{(E)-O-(1-(2-(2,6,6-Trimethylcyclohex-1-en-1-yl)vinyl)cyclopropyl)}

benzoyl(phenyl)carbamothioate (S19): Prepared according to General Proceduer D on 1.9mmol scale by stirring for $1 \mathrm{~h}$ after the addition of $\mathrm{NaH}$ and PhNCS and for $1 \mathrm{~h}$ after the addition of $\mathrm{BzCl}$. The crude residue was purified by flash column chromatography (gradient of $0-5 \%$ EtOAc/hexanes) to yield the desired product as a yellow solid $(0.34 \mathrm{~g}, 0.78 \mathrm{mmol}, 41 \%) .{ }^{1} \mathbf{H}$ NMR (500 MHz, $\left.\mathrm{CDCl}_{3}, 298 \mathrm{~K}\right): \delta_{\mathrm{H}} 7.85-7.80(\mathrm{~m}, 2 \mathrm{H}), 7.60-55(\mathrm{~m}, 1 \mathrm{H}), 7.49-7.41(\mathrm{~m}, 4 \mathrm{H})$, 7.39-7.34 (m, 1H), 7.34-7.29 (m, 2H), 5.94-5.88 (m, 1H), $5.41(\mathrm{~d}, J=16.1 \mathrm{~Hz}, 1 \mathrm{H}), 1.98-1.93$ $(\mathrm{m}, 2 \mathrm{H}), 1.65-1.62(\mathrm{~m}, 3 \mathrm{H}), 1.62-1.56(\mathrm{~m}, 2 \mathrm{H}), 1.46-1.41(\mathrm{~m}, 2 \mathrm{H}), 0.97(\mathrm{~s}, 6 \mathrm{H}), 0.90-0.84(\mathrm{~m}$, 2H), 0.70-0.66 (m, 2H) ppm; ${ }^{13} \mathbf{C}$ NMR (126 MHz, $\left.\mathrm{CDCl}_{3}, 298 \mathrm{~K}\right): \delta_{\mathrm{C}} 191.0,172.1,141.8$, 136.6, 136.0, 132.6, 130.9, 129.5, 128.9, 128.7, 128.5, 128.2, 128.2, 127.8, 64.9, 39.3, 34.1, 32.7, 28.7, 21.4, 19.3, $15.1 \mathrm{ppm}$; HRMS $m / z$ (DART): calcd for $\mathrm{C}_{28} \mathrm{H}_{32} \mathrm{NO}_{2} \mathrm{~S}(\mathrm{M}+\mathrm{H}): 446.2148$; found: 446.2157; IR (neat): 3038, 2955, 2927, 2862, 2823, 1688, 1598, 1494, 1450, 1318, 1267 , 
$1164,1072,975,695,659 \mathrm{~cm}^{-1}$; m.p.: $=76-78^{\circ} \mathrm{C} ; \mathbf{R}_{\mathbf{f}}(8: 2$ hexanes/toluene; $\mathrm{UV} / p$-anisaldehyde): 0.30 .<smiles>CCC1CC1OC(=S)N([18O])c1ccccc1</smiles>

$O$-((cis)-2-Ethyl-1-phenylcyclopropyl) benzoyl(phenyl)carbamothioate (cis-17): Prepared on 4.0-mmol scale by stirring for $30 \mathrm{~min}$ after the addition of $\mathrm{NaH}$ and $\mathrm{PhNCS}$ and for $16 \mathrm{~h}$ after the addition of $\mathrm{BzCl}$. The crude material was purified by flash column chromatography (gradient of 0-20\% EtOAc/hexanes), then stripped with hexanes and washed with hexanes to yield the product as a pale yellow solid (1.4 g, $3.5 \mathrm{mmol}, 88 \%) .{ }^{1} \mathbf{H} \mathbf{~ N M R}\left(500 \mathrm{MHz}, \mathrm{CDCl}_{3}, 298 \mathrm{~K}\right): \delta_{\mathrm{H}}$ 7.92-7.85 (m, 2H), 7.59-7.52 (m, 1H), 7.46-7.38 (m, 4H), 7.36-7.25 (m, 5H), 7.24-7.18 (m, $1 \mathrm{H}), 7.17-7.11(\mathrm{~m}, 2 \mathrm{H}), 1.80-1.69(\mathrm{~m}, 1 \mathrm{H}), 1.31-1.17(\mathrm{~m}, 1 \mathrm{H}), 1.16-1.05(\mathrm{~m}, 1 \mathrm{H}), 1.05-0.94$ (m, 2H), 0.82-0.76 (m, 3H) ppm; ${ }^{13} \mathbf{C}$ NMR (125 MHz, $\left.\mathrm{CDCl}_{3}, 298 \mathrm{~K}\right): \delta_{\mathrm{C}} 190.8,172.2,141.1$, 140.2, 135.0, 133.1, 129.7, 129.5, 128.9, 128.4, 128.3, 128.2, 127.0, 126.4, 69.3, 30.6, 22.0, 20.5, 13.8; HRMS $m / z$ (DART): calcd for $\mathrm{C}_{25} \mathrm{H}_{24} \mathrm{NO}_{2} \mathrm{~S}(\mathrm{M}+\mathrm{H}$ ): 402.1522; found: 402.1536; IR (neat): 2970, 1686, 1494, 1449, 1318, 1274, 1175, 1017, 755, $694 \mathrm{~cm}^{-1}$; m.p.: $71-73{ }^{\circ} \mathrm{C} ; \mathbf{R}_{\mathbf{f}}(9: 1$ hexanes/EtOAc; UV): 0.45.<smiles>CCC1C[C@@H]1OC(=S)N([18O])c1ccccc1</smiles>

$O$-(trans-2-Ethyl-1-phenylcyclopropyl) benzoyl(phenyl)carbamothioate (trans-17): Prepared on 1.4-mmol scale by stirring for $30 \mathrm{~min}$ after the addition of $\mathrm{NaH}$ and $\mathrm{PhNCS}$ and for $16 \mathrm{~h}$ after the addition of $\mathrm{BzCl}$. The crude residue was purified by flash column chromatography (gradient of $0-30 \% \mathrm{Et}_{2} \mathrm{O} /$ hexanes) and the fractions containing product were collected, concentrated, stripped with hexanes and recrystallized (hexanes) to yield the product as a white solid $(0.35 \mathrm{~g}$, $0.87 \mathrm{mmol}, 62 \%) .{ }^{1} \mathbf{H}$ NMR $\left(500 \mathrm{MHz}, \mathrm{CDCl}_{3}, 298 \mathrm{~K}\right): \delta_{\mathrm{H}} 7.83-7.78(\mathrm{~m}, 2 \mathrm{H}), 7.60-7.55(\mathrm{~m}$, 1H), 7.47-7.41 (m, 2H), 7.41-7.35 (m, 2H), 7.35-7.30 (m, 1H), 7.29-7.20 (m, 7H), 1.24-1.16 $(\mathrm{m}, 2 \mathrm{H}), 1.07-0.96(\mathrm{~m}, 1 \mathrm{H}), 0.87-0.76(\mathrm{~m}, 1 \mathrm{H}), 0.74-0.61(\mathrm{~m}, 4 \mathrm{H}) \mathrm{ppm} ;{ }^{13} \mathbf{C}$ NMR $(125 \mathrm{MHz}$, $\left.\mathrm{CDCl}_{3}, 298 \mathrm{~K}\right): \delta_{\mathrm{C}} 190.8,172.4,141.7,136.1,135.8,132.7,129.5,129.5,128.9,128.8,128.4$, 128.2, 127.9, 127.8, 70.6, 27.6, 22.2, 18.0, $13.0 \mathrm{ppm}$; HRMS $m / z$ (DART): calcd for $\mathrm{C}_{25} \mathrm{H}_{24} \mathrm{NO}_{2} \mathrm{~S}(\mathrm{M}+\mathrm{H}): 402.1522$; found: 402.1527; IR (neat): 3062, 2980, 1705, 1683, 1595 , 1491, 1450, 1317, 1271, 1204, 1174, 1059, 1022, 856, 752, $694 \mathrm{~cm}^{-1}$; m.p.: $119-120{ }^{\circ} \mathrm{C} ; \mathbf{R}_{\mathbf{f}}(9: 1$ hexanes/EtOAc; UV): 0.41.<smiles>CC1CC1OC(=S)N(Cc1ccccc1)c1ccccc1</smiles>

$O$-((cis)-2-methyl-1-phenylcyclopropyl) benzoyl(phenyl)carbamothioate (cis-18): Prepared on 4.0-mmol scale by stirring for $30 \mathrm{~min}$ after the addition of $\mathrm{NaH}$ and $\mathrm{PhNCS}$ and for $16 \mathrm{~h}$ after the addition of $\mathrm{BzCl}$. The crude material was purified by flash column chromatography (gradient of $0-20 \%$ EtOAc/hexanes), then washed with hexanes to yield the product as a white solid (1.5 g, 3.9 mmol, 98\%). ${ }^{1} \mathbf{H}$ NMR $\left(500 \mathrm{MHz}, \mathrm{CDCl}_{3}, 298 \mathrm{~K}\right): \delta_{\mathrm{H}} 7.91-7.86(\mathrm{~m}, 2 \mathrm{H}), 7.58-7.52(\mathrm{~m}$, $1 \mathrm{H}), 7.46-7.37(\mathrm{~m}, 4 \mathrm{H}), 7.46-7.31(\mathrm{~m}, 3 \mathrm{H}), 7.30-7.25(\mathrm{~m}, 2 \mathrm{H}), 7.23-7.18(\mathrm{~m}, 1 \mathrm{H}), 7.15-7.10$ $(\mathrm{m}, 2 \mathrm{H}), 1.63(\mathrm{dd}, J=9.6,6.9 \mathrm{~Hz}, 1 \mathrm{H}), 1.29-1.18(\mathrm{~m}, 1 \mathrm{H}), 0.98(\mathrm{~d}, J=6.3 \mathrm{~Hz}, 3 \mathrm{H}), 0.94(\mathrm{dd}, J$ $=7.9,6.9 \mathrm{~Hz}, 1 \mathrm{H}) \mathrm{ppm} ;{ }^{13} \mathbf{C}$ NMR $\left(125 \mathrm{MHz}, \mathrm{CDCl}_{3}, 298 \mathrm{~K}\right): \delta_{\mathrm{C}} 191.0,172.2,141.2,140.2$, 
135.2, 133.0, 129.6, 129.5, 128.9, 128.4, 128.4, 128.2, 126.9, 126.2, 69.3, 23.0, 21.8, 13.1;

HRMS $m / z$ (DART): calcd for $\mathrm{C}_{24} \mathrm{H}_{22} \mathrm{NO}_{2} \mathrm{~S}$ (M+H): 388.1366; found: 388.1371; IR (neat): $3163,3035,2926,1694,1678,1595,1493,1274,1238,1172,1074,1004,853,743,691 \mathrm{~cm}^{-1}$; m.p.: $90-91^{\circ} \mathrm{C} ; \mathbf{R}_{\mathbf{f}}(9: 1$ hexanes/EtOAc; UV): 0.46.<smiles>[Y17]C(=S)NC(=S)OC(C)c1ccc2ccccc2c1</smiles>

$O$-(1-(Naphthalen-2-yl)ethyl) benzoyl(phenyl)carbamothioate (racemic) (rac-15): Prepared on 5.0-mmol scale by stirring for $30 \mathrm{~min}$ after the addition of $\mathrm{NaH}$ and PhNCS and for $16 \mathrm{~h}$ after the addition of $\mathrm{BzCl}$. The crude material was purified by flash column chromatography (gradient of $0-20 \%$ EtOAc/hexanes), then concentrated and washed with $\mathrm{Et}_{2} \mathrm{O}$ to yield the product as a white solid (1.1 g, $2.7 \mathrm{mmol}, 54 \%) .{ }^{1} \mathbf{H}$ NMR $\left(500 \mathrm{MHz}, \mathrm{CDCl}_{3}, 298 \mathrm{~K}\right): \delta_{\mathrm{H}} 7.87-7.83(\mathrm{~m}, 2 \mathrm{H})$, 7.82-7.78 (m, 1H), 7.76-7.72 (m, 1H), $7.70($ app d, $J=8.5 \mathrm{~Hz}, 1 \mathrm{H}), 7.50-7.46(\mathrm{~m}, 3 \mathrm{H}), 7.45-$ $7.30(\mathrm{~m}, 8 \mathrm{H}), 7.09(\mathrm{dd}, J=8.5,1.8 \mathrm{~Hz}, 1 \mathrm{H}), 6.48(\mathrm{q}, J=6.6 \mathrm{~Hz}, 1 \mathrm{H}), 1.39(\mathrm{~d}, J=6.6 \mathrm{~Hz}, 3 \mathrm{H})$ ppm; ${ }^{13} \mathbf{C}$ NMR $\left(125 \mathrm{MHz}, \mathrm{CDCl}_{3}, 298 \mathrm{~K}\right): \delta_{\mathrm{C}} 191.0,172.3,141.6,137.3,135.6,133.2,133.1$, $132.9,129.6,129.0,128.8,128.5,128.5,128.4,128.2,127.8,126.4,126.4,125.7,124.2,81.5$, $21.5 \mathrm{ppm}$; HRMS $\mathrm{m} / z$ (DART): calcd for $\mathrm{C}_{26} \mathrm{H}_{22} \mathrm{NO}_{2} \mathrm{~S}(\mathrm{M}+\mathrm{H})$ : 412.1366; found: 412.1367; IR (neat): 2983, 1683, 1601, 1486, 1451, 1341, 1301, 1267, 1213, 1044, 1006, 853, 821, 749, 691 $\mathrm{cm}^{-1}$; m.p.: $100-102{ }^{\circ} \mathrm{C} ; \mathbf{R}_{\mathbf{f}}(9: 1$ hexanes/EtOAc; UV): 0.43 .<smiles>[Y9]C(NC(=S)Nc1ccccc1)c1ccc2ccccc2c1</smiles>

$O$-(1-(Naphthalen-2-yl)ethyl) benzoyl(phenyl)carbamothioate (enantioenriched) (15):

Prepared on 0.41-mmol scale by stirring for $30 \mathrm{~min}$ after the addition of $\mathrm{NaH}$ and $\mathrm{PhNCS}$ and for $16 \mathrm{~h}$ after the addition of $\mathrm{BzCl}$. The crude material was purified by flash column chromatography (gradient of 0-20\% EtOAc/hexanes), then concentrated and washed with hexanes to yield the product as a white solid $(0.11 \mathrm{~g}, 0.27 \mathrm{mmol}, 66 \%)$. The analytical data was identical to that of the racemic product. Chiral HPLC (Chiralpak IA, $0.3 \% i-\mathrm{PrOH} /$ hexanes, $1.0 \mathrm{~mL} / \mathrm{min}, \lambda=224$ $\mathrm{nm}$ ): $t_{\mathrm{R}}$ (major): $8.54 \mathrm{~min} ; t_{\mathrm{R}}$ (minor): $9.23 \mathrm{~min} ; 83 \%$ e.e. 

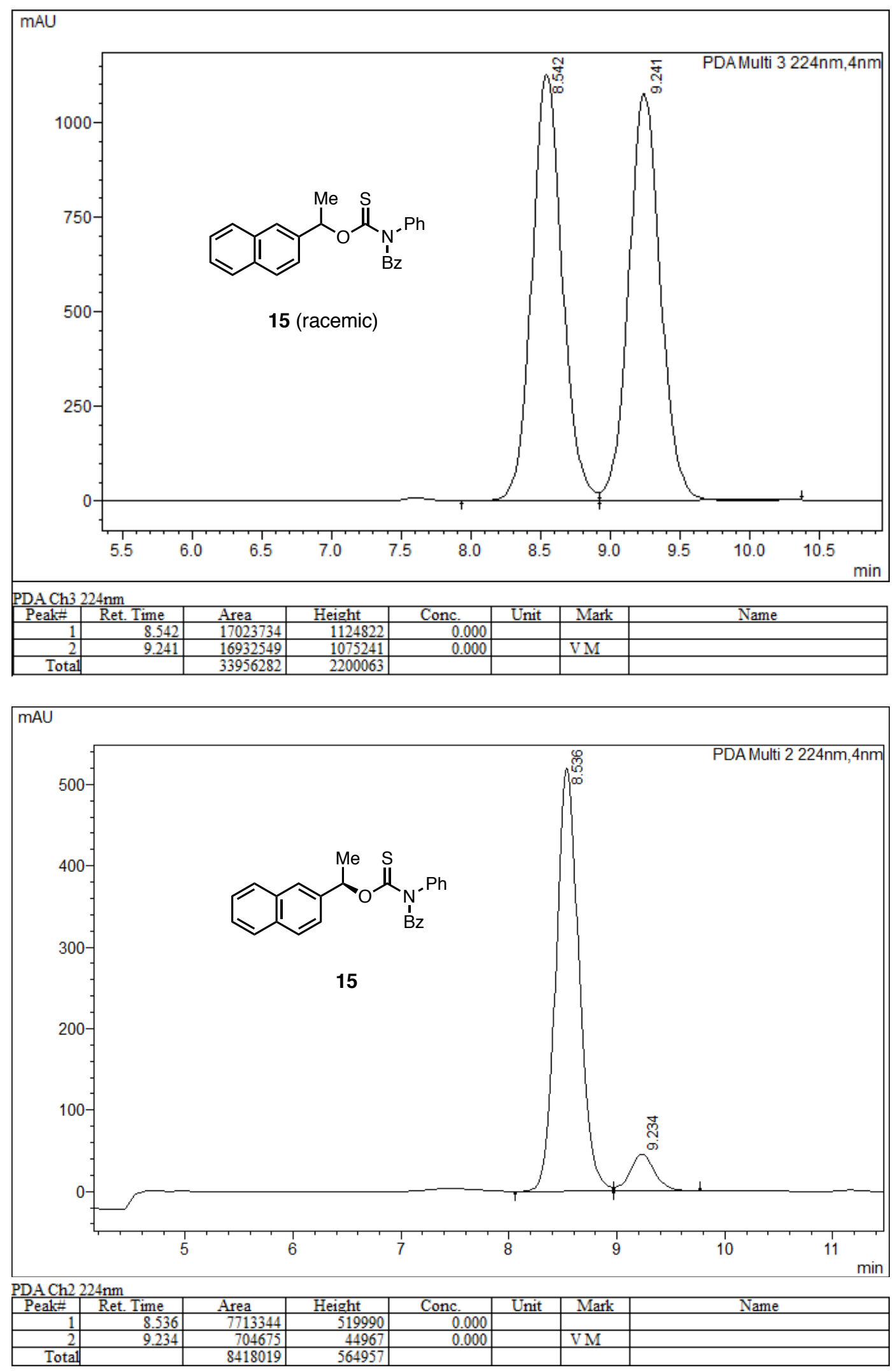


\section{E.3. Preparation of alcohols}

The following cyclopropanols were prepared as previously described: ${ }^{8} 1$-phenylcyclopropanol, 1-(4-fluorophenyl)cyclopropan-1-ol, 1-benzylcyclopropanol, 1-([1,1'-biphenyl]-4-

yl)cyclopropan-1-ol, 1-(thiophen-2-yl)cyclopropan-1-ol, (E)-1-styrylcyclopropan-1-ol, 6,6adihydrocyclopropa[a]inden-1a(1H)-ol, 1-benzylcyclopropanol.

General Procedure E: Simmons-Smith sequence for the synthesis of cyclopropanols

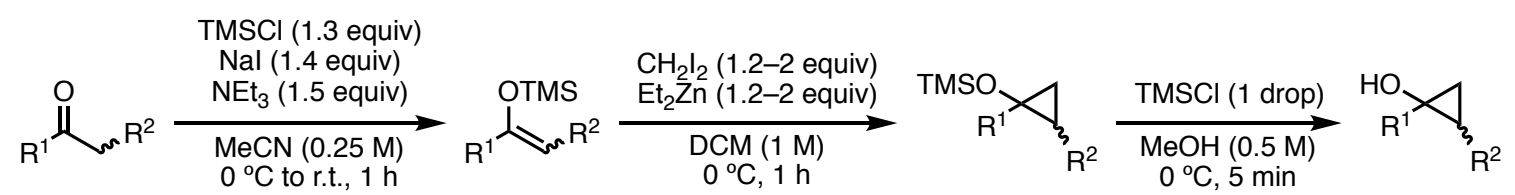

Silyl enol ether (Step 1): To an appropriately sized flask with a stir bar was added sodium iodide (1.4 equiv) and the flask was flame-dried and cooled under vacuum. The flask was backfilled with $\mathrm{N}_{2}$ and $\mathrm{MeCN}(0.25 \mathrm{M})$ was added, followed by ketone substrate (1.0 equiv). The solution was cooled to $0{ }^{\circ} \mathrm{C}$ and stirred, and chlorotrimethylsilane (1.3 equiv) and triethylamine (1.5 equiv) were sequentially added. The reaction was stirred at $r . t$. for $1 \mathrm{~h}$. The reaction was concentrated under vacuum and the solid concentrate was extracted with hexanes $(\times 3)$ (for more polar substrates $\mathrm{Et}_{2} \mathrm{O}$ can be used). The organic extracts were combined and concentrated to yield the crude silyl enol ether, which was used in the next step without further purification.

TMS-protected cyclopropanol (Step 2): The crude material was transferred to an appropriately sized flame-dried round-bottom flask and a stir bar was added. The flask was evacuated and backfilled with $\mathrm{N}_{2}(\times 3)$ and DCM $(1.0 \mathrm{M})$ was added. The solution was cooled to $0{ }^{\circ} \mathrm{C}$ and diiodomethane (1.2-2.0 equiv) was added. Over $10 \mathrm{~min}$, diethylzinc (1.0 M solution in hexanes, 1.2-2.0 equiv) was added down the side of the flask. The reaction was stirred at $0{ }^{\circ} \mathrm{C}$ for $1 \mathrm{~h}$. The reaction was slowly quenched with sat. aq. $\mathrm{NaHCO}_{3}$ (gas evolves). The mixture was extracted with DCM $(\times 3)$ and the organic fractions were combined, washed with $\mathrm{H}_{2} \mathrm{O}(\times 1)$ and brine $(\times 1)$, dried over $\mathrm{MgSO}_{4}$, and concentrated to yield the crude material, which was used in the next step without further purification. If incomplete conversion of the silyl enol ether was determined by ${ }^{1} \mathrm{H}$ NMR, the crude material can be re-exposed to Simmons-Smith conditions.

Cyclopropanol (Step 3): The crude material from the previous step was dissolved in $\mathrm{MeOH}$ $(0.5 \mathrm{M})$ (note: anhydrous $\mathrm{MeOH}$ is necessary) and the solution was cooled to $0{ }^{\circ} \mathrm{C}$. TMSCl ( 1 drop) was added and the solution was stirred at $0{ }^{\circ} \mathrm{C}$ for $5 \mathrm{~min}$. The solution was concentrated and the crude concentrate was purified by flash column chromatography to yield the cyclopropanol. As a precaution, all cyclopropanols were stored at $-20^{\circ} \mathrm{C}$.<smiles>OC1(c2ccco2)CC1</smiles>

1-(Furan-2-yl)cyclopropan-1-ol (S20): Prepared according to General Procedure E on 8.0mmol scale from 1-(furan-2-yl)ethan-1-one ( $0.88 \mathrm{~g}, 8.0 \mathrm{mmol}, 1.0 \mathrm{equiv})$. The Simmons-Smith reaction was performed using diiodomethane $(1.3 \mathrm{~mL}, 16 \mathrm{mmol}, 2.0$ equiv) and diethylzinc (16 $\mathrm{mL}, 16 \mathrm{mmol}$ ). The cyclopropanol was purified by flash column chromatography (gradient of 0 
$30 \% \mathrm{EtOAc} / \mathrm{hexanes})$ to yield the product as a pale yellow oil $(0.39 \mathrm{~g}, 3.1 \mathrm{mmol}, 39 \%$ over three steps). Analytical data: ${ }^{1} \mathbf{H}$ NMR (400 MHz, $\left.\mathrm{CDCl}_{3}, 298 \mathrm{~K}\right): \delta_{\mathrm{H}} 7.33-7.29(\mathrm{~m}, 1 \mathrm{H}), 6.34-6.19$ (m, 1H), 6.23-6.19 (m, 1H), 2.82 (br s, 1H), 1.18-1.11 (m, 2H), 1.10-1.02 (m, 2H) ppm; ${ }^{13} \mathbf{C}$ NMR $\left(100 \mathrm{MHz}, \mathrm{CDCl}_{3}, 298 \mathrm{~K}\right): \delta_{\mathrm{C}} 156.7,141.5,110.5,104.9,52.4,15.3 \mathrm{ppm} ; \mathbf{R}_{\mathbf{f}}(9: 1$ hexanes/EtOAc; $\left.\mathrm{KMnO}_{4}\right): 0.17$.<smiles>OC1(c2ccc(Cl)cc2)CC1</smiles>

1-(4-Chlorophenyl)cyclopropan-1-ol (S21): Prepared according to General Procedure E on 8.0mmol scale from 4-chloroacetophenone (1.0 mL, $8.0 \mathrm{mmol}, 1.0$ equiv). The Simmons-Smith reaction was performed using diiodomethane $(0.96 \mathrm{~mL}, 12 \mathrm{mmol}, 1.5$ equiv) and diethylzinc (12 $\mathrm{mL}, 12 \mathrm{mmol}, 1.5$ equiv). The cyclopropanol was purified by flash column chromatography (gradient of 0-20\% EtOAc/hexanes) to yield the product as a white solid (0.44 g, $2.6 \mathrm{mmol}, 33 \%$ over three steps). Analytical data: $\left.{ }^{10}{ }^{1} \mathbf{H ~ N M R ~ ( 4 0 0 ~ M H z , ~} \mathrm{CDCl}_{3}, 298 \mathrm{~K}\right): \delta_{\mathrm{H}} 7.32-7.26(\mathrm{~m}, 2 \mathrm{H})$, 7.25-7.20 (m, 2H), 2.39 (br s, 1H), 1.34-1.20 (m, 2H), 1.08-0.95 (m, 2H) ppm; ${ }^{13}$ C NMR (100 $\left.\mathrm{MHz}, \mathrm{CDCl}_{3}, 298 \mathrm{~K}\right): \delta_{\mathrm{C}} 143.0,132.3,128.6,126.0,56.4,18.2 \mathrm{ppm} ; \mathbf{R}_{\mathbf{f}}(8: 2$ hexanes/EtOAc; $\left.\mathrm{UV} / \mathrm{KMnO}_{4}\right): 0.53$.<smiles>OC1(c2ccc(C(F)(F)F)cc2)CC1</smiles>

1-(4-(Trifluoromethyl)phenyl)cyclopropan-1-ol (S22): Prepared according to General Procedure E on 8.0-mmol scale using 1-(4-(trifluoromethyl)phenyl)ethan-1-one (1.3 mL, 8.0 mmol, 1.0 equiv). The Simmons-Smith reaction was performed using diiodomethane $(0.77 \mathrm{~mL}$, $9.6 \mathrm{mmol}, 1.2$ equiv) and diethylzinc $(9.6 \mathrm{~mL}, 9.6 \mathrm{mmol}, 1.2$ equiv). The cyclopropanol was purified by flash column chromatography (gradient of 5-30\% EtOAc/hexanes) to yield the product as a white solid ( $0.36 \mathrm{~g}, 1.8 \mathrm{mmol}, 23 \%$ over three steps $)$. Analytical data: ${ }^{11}{ }^{1} \mathbf{H}$ NMR $\left(400 \mathrm{MHz}, \mathrm{CDCl}_{3}, 298 \mathrm{~K}\right): \delta_{\mathrm{H}} 7.60-7.53(\mathrm{~m}, 2 \mathrm{H}), 7.40-7.33(\mathrm{~m}, 2 \mathrm{H}), 2.60$ (br s, $\left.1 \mathrm{H}\right), 1.38-1.30$ (m, 2H), 1.13-1.06 (m, 2H) ppm; ${ }^{19} \mathbf{F}$ NMR $\left(377 \mathrm{MHz}, \mathrm{CDCl}_{3}, 298 \mathrm{~K}\right): \delta_{\mathrm{F}}-62.4 \mathrm{ppm} ;{ }^{13} \mathbf{C}$ NMR $\left(125 \mathrm{MHz}, \mathrm{CDCl}_{3}, 298 \mathrm{~K}\right): \delta_{\mathrm{C}} 148.8(\mathrm{q}, J=1.4 \mathrm{~Hz}), 128.6(\mathrm{q}, J=32.2 \mathrm{~Hz}), 125.4(\mathrm{q}, J=3.8$ $\mathrm{Hz}), 124.4$ (q, $J=261.5 \mathrm{~Hz}), 124.3,56.3,19.1 \mathrm{ppm} ; \mathbf{R}_{\mathbf{f}}\left(9: 1\right.$ hexanes/EtOAc; $\left.\mathrm{UV} / \mathrm{KMnO}_{4}\right): 0.33$.<smiles>OC12CCC1c1ccccc1OC2</smiles>

(trans)-1a,2-Dihydrocyclopropa[c]chromen-7b(1H)-ol (S23): Prepared according to General Procedure E on 8.0-mmol scale from 4-chromanone (1.2 g, $8.0 \mathrm{mmol}, 1.0$ equiv). The SimmonsSmith reaction was performed using diiodomethane $(0.77 \mathrm{~mL}, 9.6 \mathrm{mmol}, 1.2$ equiv $)$ and diethylzinc ( $9.6 \mathrm{~mL}, 9.6 \mathrm{mmol}, 1.2$ equiv). The cyclopropanol was purified by flash column chromatography (gradient of $0-40 \%$ EtOAc/hexanes) to yield the product as a colourless oil (0.75 g, $4.6 \mathrm{mmol}, 58 \%$ over three steps). ${ }^{1} \mathbf{H}$ NMR (400 MHz, $\left.\mathrm{CDCl}_{3}, 298 \mathrm{~K}\right): \delta_{\mathrm{H}} 7.64-7.58(\mathrm{~m}$, $1 \mathrm{H}), 7.13(\operatorname{app~td}, J=7.8,1.8 \mathrm{~Hz}, 1 \mathrm{H}), 7.02(\mathrm{app} \mathrm{tt}, J=7.5,1.4 \mathrm{~Hz}, 1 \mathrm{H}), 6.84(\mathrm{dd}, J=8.0,1.2$ $\mathrm{Hz}, 1 \mathrm{H}), 4.23-4.18(\mathrm{~m}, 1 \mathrm{H}), 3.91-3.85$ (m, 1H), 2.50 (br s, 1H), 1.95-1.85 (m, 1H), 1.44-1.35 $(\mathrm{m}, 1 \mathrm{H}), 1.26-1.19(\mathrm{~m}, 1 \mathrm{H}) \mathrm{ppm} ;{ }^{13} \mathbf{C} \mathbf{N M R}\left(100 \mathrm{MHz}, \mathrm{CDCl}_{3}, 298 \mathrm{~K}\right): \delta_{\mathrm{C}} 151.1,129.7,127.2$, $124.5,121.9,117.1,62.4,52.0,27.4,18.3 \mathrm{ppm}$. 
<smiles>OC12CCCc3ccccc3CC1C2</smiles>

(trans)-1,1a,2,3-Tetrahydro-7b $H$-cyclopropa[a]naphthalen-7b-ol (S24): Prepared according to General Procedure E on 8.0-mmol scale from tetralone ( $1.1 \mathrm{~mL}, 8.0 \mathrm{mmol}, 1.0$ equiv). The Simmons-Smith reaction was performed using diiodomethane $(0.96 \mathrm{~mL}, 12 \mathrm{mmol}, 1.5 \mathrm{equiv})$ and diethylzinc (12 mL, $12 \mathrm{mmol}, 1.5$ equiv). The cyclopropanol was purified by flash column chromatography (gradient of $0-30 \%$ EtOAc/hexanes) to yield the product as a white solid (0.88 g, $5.5 \mathrm{mmol}, 69 \%$ over three steps). ${ }^{1} \mathbf{H}$ NMR $\left(400 \mathrm{MHz}, \mathrm{CDCl}_{3}, 298 \mathrm{~K}\right): \delta_{\mathrm{H}} 7.75$ (dd, $J=$ 7.7, $1.4 \mathrm{~Hz}, 1 \mathrm{H}), 7.30$ (app tt, $J=7.4,1.3 \mathrm{~Hz}, 1 \mathrm{H}), 7.17$ (app td, $J=7.4,1.4 \mathrm{~Hz}, 1 \mathrm{H}), 7.12-7.07$ (m, 1H), 2.72-2.61 (m, 1H), 2.55-2.32 (m, 2H), 2.09-1.98 (m, 1H), 1.85-1.72 (m, 2H), 1.26 (dd, $J=9.8,5.7 \mathrm{~Hz}, 1 \mathrm{H}), 1.11(\mathrm{dd}, J=5.8,5.8 \mathrm{~Hz}, 1 \mathrm{H}) \mathrm{ppm} ;{ }^{13} \mathbf{C}$ NMR $\left(100 \mathrm{MHz}, \mathrm{CDCl}_{3}, 298 \mathrm{~K}\right)$ : $\delta_{\mathrm{C}} 140.6,132.8,128.2,126.3,125.6,124.1,54.6,26.1,24.6,18.4,16.4$ ppm; $\mathbf{R}_{\mathbf{f}}(9: 1$ hexanes/EtOAc; $\left.\mathrm{UV} / \mathrm{KMnO}_{4}\right): 0.22$.

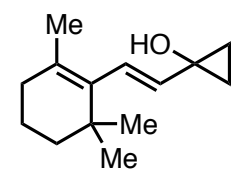

(E)-1-(2-(2,6,6-Trimethylcyclohex-1-en-1-yl)vinyl)cyclopropan-1-ol (S25): Prepared according to General Procedure E on 15-mmol scale from $\beta$-ionone $(3.1 \mathrm{~mL}, 15 \mathrm{mmol}, 1.0$ equiv). The Simmons-Smith reaction was performed using diiodomethane $(1.7 \mathrm{~mL}, 21 \mathrm{mmol}$, 1.4 equiv) and diethylzinc ( $21 \mathrm{~mL}$ of a $1.0 \mathrm{M}$ solution in hexanes, $21 \mathrm{mmol}, 1.4$ equiv). The cyclopropanol was purified by flash column chromatography (gradient of 0-5\% EtOAc/hexanes) to yield the desired product as a yellow solid (0.98 g, $4.7 \mathrm{mmol}, 32 \%$ over three steps). ${ }^{1} \mathbf{H}$ NMR $\left(400 \mathrm{MHz}, \mathrm{CDCl}_{3}, 298 \mathrm{~K}\right): \delta_{\mathrm{H}} 6.10(\mathrm{dtd}, J=15.9,2.0,1.0 \mathrm{~Hz}, 1 \mathrm{H}), 5.24(\mathrm{~d}, J=16.0 \mathrm{~Hz}, 1 \mathrm{H})$, 2.00-1.95 (m, 2H), $1.67(\mathrm{q}, J=1.0 \mathrm{~Hz}, 3 \mathrm{H}), 1.60(\mathrm{ddt}, \mathrm{J}=9.9,6.4,3.4 \mathrm{~Hz}, 2 \mathrm{H}), 1.47-1.43(\mathrm{~m}$, 2H), 1.07-1.03 (m, 2H), 1.00 (s, 6H), 0.78-0.70 (m, 2H) ppm; ${ }^{13} \mathbf{C}$ NMR (100 MHz, CDCl, 298 $\mathrm{K}): \delta_{\mathrm{C}} 137.1,137.0,128.2,123.9,55.8,39.5,34.1,32.8,28.7,21.4,19.3,15.9 \mathrm{ppm} ; \mathbf{H R M S ~} \mathrm{m} / \mathrm{z}$ (DART): calcd for $\mathrm{C}_{14} \mathrm{H}_{23} \mathrm{O}(\mathrm{M}+\mathrm{H}): 207.1743$; found: 207.1748; IR (neat): 3236 (broad), 3089, 2982, 2965, 2925, 2905, 2863, 1357, 1290, 1018, 979, 965, 919, 886, $679 \mathrm{~cm}^{-1}$; m.p.: 50-53 ${ }^{\circ}$; $\mathbf{R}_{\mathbf{f}}(19: 1$ hexanes/EtOAc; $p$-anisaldehyde): 0.30 .<smiles>CCC1(O)CC1[Pb]1(c2ccccc2)CC1</smiles>

(cis)-2-Ethyl-1-phenylcyclopropan-1-ol (>20:1 d.r.) (S26): Prepared according to General Procedure E on 12-mmol scale using butyrophenone (1.7 mL, $12 \mathrm{mmol}, 1.0$ equiv). The Simmons-Smith reaction was performed using diiodomethane (1.1 mL, $14 \mathrm{mmol}, 1.2$ equiv) and diethylzinc (14 mL, 14 mmol, 1.2 equiv). The crude cyclopropanol was a colourless oil, which was sufficiently clean to use without further purification $(1.3 \mathrm{~g}, 8.0 \mathrm{mmol}, 67 \%$ over three steps). The relative configuration was determined by the configuration of the silyl enol ether precursor (see below). Analytical data: ${ }^{12}{ }^{1} \mathbf{H}$ NMR (400 MHz, $\left.\mathrm{CDCl}_{3}, 298 \mathrm{~K}\right): \delta_{\mathrm{H}} 7.37-7.27(\mathrm{~m}, 4 \mathrm{H}), 7.24$ $7.18(\mathrm{~m}, 1 \mathrm{H}), 2.07$ (br s, 1H), 1.75-1.59 (m, 2H), 1.23-1.12 (m, 2H), $1.08(\mathrm{t}, J=7.4 \mathrm{~Hz}, 3 \mathrm{H})$, $0.88-0.83(\mathrm{~m}, 1 \mathrm{H}) \mathrm{ppm} ;{ }^{13} \mathrm{C}$ NMR $\left(100 \mathrm{MHz}, \mathrm{CDCl}_{3}, 298 \mathrm{~K}\right): \delta_{\mathrm{C}} 145.6,128.4,126.3,124.3$, 59.7, 30.8, 22.9, 21.4, 14.4 ppm; $\mathbf{R}_{\mathbf{f}}\left(9: 1\right.$ hexanes/EtOAc; $\left.\mathrm{UV} / \mathrm{KMnO}_{4}\right): 0.35$. 


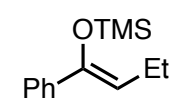

(Z)-Trimethyl((1-phenylbut-1-en-1-yl)oxy)silane (>20:1 isomer ratio) (S27): ${ }^{13}$ Colourless oil. ${ }^{1} \mathbf{H}$ NMR (400 MHz, $\left.\mathrm{CDCl}_{3}, 298 \mathrm{~K}\right): \delta_{\mathrm{H}} 7.50-7.43(\mathrm{~m}, 2 \mathrm{H}), 7.33-7.18(\mathrm{~m}, 3 \mathrm{H}), 7.24(\mathrm{t}, J=7.1$ $\mathrm{Hz}, 1 \mathrm{H}), 2.22$ (pent, $J=7.4 \mathrm{~Hz}, 2 \mathrm{H}), 1.04(\mathrm{td}, J=7.6,0.5 \mathrm{~Hz}, 3 \mathrm{H}), 0.13(\mathrm{~s}, 9 \mathrm{H}) \mathrm{ppm} ;{ }^{13} \mathbf{C} \mathbf{N M R}$ $\left(100 \mathrm{MHz}, \mathrm{CDCl}_{3}, 298 \mathrm{~K}\right): \delta_{\mathrm{C}} 148.5,139.4,128.1,127.4,125.5,113.5,19.7,14.4,0.7 \mathrm{ppm} ; \mathbf{R}_{\mathbf{f}}$ (5\% EtOAc/hexanes; UV/KMnO 4$): 0.78$.

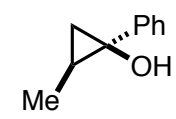

(cis)-2-Methyl-1-phenylcyclopropan-1-ol (>20:1 d.r.) (S28): Prepared according to General Procedure E on 10-mmol scale using propiophenone (1.3 mL, $10 \mathrm{mmol}, 1.0$ equiv). The Simmons-Smith reaction was performed using diiodomethane $(0.96 \mathrm{~mL}, 12 \mathrm{mmol}, 1.2$ equiv) and diethylzinc (12 mL, $12 \mathrm{mmol}, 1.2$ equiv). The cyclopropanol was purified by flash column chromatography (gradient of 0-20\% EtOAc/hexanes) to yield the product as a colourless oil which solidified over time to form a white solid $(0.95 \mathrm{~g}, 6.4 \mathrm{mmol}, 64 \%$ over three steps $)$. The relative configuration was determined by the configuration of the silyl enol ether precursor (see below). Analytical data: ${ }^{1} \mathbf{H}$ NMR $\left(500 \mathrm{MHz}, \mathrm{CDCl}_{3}, 298 \mathrm{~K}\right): \delta_{\mathrm{H}} 7.36-7.31(\mathrm{~m}, 2 \mathrm{H}), 7.30-7.26$ (m, 2H), 7.24-7.19 (m, 1H), 2.28 (br s, 1H), 1.35-1.31 (m, 3H), 1.29-1.20 (m, 2H), 0.85-0.82 (m, 1H) ppm; ${ }^{13} \mathbf{C}$ NMR $\left(125 \mathrm{MHz}, \mathrm{CDCl}_{3}, 298 \mathrm{~K}\right): \delta_{\mathrm{C}} 145.6,128.4,126.2,124.0,59.2,24.3$, 23.3, 12.7 ppm; $\mathbf{R}_{\mathbf{f}}\left(9: 1\right.$ hexanes/EtOAc; $\left.\mathrm{UV} / \mathrm{KMnO}_{4}\right): 0.28$.<smiles>COC(=C[As])c1ccccc1</smiles>

(Z)-Trimethyl((1-phenylprop-1-en-1-yl)oxy)silane (>20:1 isomer ratio) (S29): ${ }^{14}$ Colourless oil. ${ }^{1} \mathbf{H}$ NMR $\left(400 \mathrm{MHz}, \mathrm{CDCl}_{3}, 298 \mathrm{~K}\right): \delta_{\mathrm{H}} 7.49-7.42(\mathrm{~m}, 2 \mathrm{H}), 7.33-7.18(\mathrm{~m}, 3 \mathrm{H}), 5.33(\mathrm{q}, J=$ $6.8 \mathrm{~Hz}, 1 \mathrm{H}), 1.74(\mathrm{~d}, J=6.8 \mathrm{~Hz}, 3 \mathrm{H}), 0.14(\mathrm{~s}, 9 \mathrm{H}) \mathrm{ppm} ;{ }^{13} \mathbf{C} \mathbf{N M R}\left(100 \mathrm{MHz}, \mathrm{CDCl}_{3}, 298 \mathrm{~K}\right): \delta_{\mathrm{C}}$ 150.0, 139.3, 128.2, 127.4, 125.3, 105.5, 11.8, 0.7 ppm; $\mathbf{R}_{\mathbf{f}}(5 \%$ EtOAc/hexanes; UV/KMnO 4$):$ 0.58 .

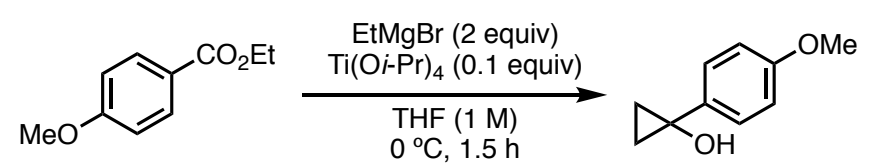

1-(4-Methoxyphenyl)cyclopropan-1-ol (S30): To a flame-dried 250-mL flask with a stir bar were added THF (40 mL, 1.0 M), ethyl 4-methoxybenzoate ( $6.5 \mathrm{~mL}, 40 \mathrm{mmol}, 1.0$ equiv), and titanium(IV) isopropoxide ( $1.2 \mathrm{~mL}, 4.0 \mathrm{mmol}, 0.10$ equiv), and the solution was stirred and cooled to $0{ }^{\circ} \mathrm{C}$. Over $1.5 \mathrm{~h}$, ethylmagnesium bromide $(84 \mathrm{~mL}$ of a $0.95 \mathrm{M}$ solution in THF, $80 \mathrm{mmol}, 2.0$ equiv) was added portionwise ( 6 portions) down the side of the flask. The reaction was quenched with $10 \% \mathrm{H}_{2} \mathrm{SO}_{4}$ and extracted with $\mathrm{Et}_{2} \mathrm{O}(\times 3)$, and the organic fractions were combined, washed with brine $(\times 1)$, dried over $\mathrm{MgSO}_{4}$, and concentrated. The crude residue was purified by flash column chromatography (gradient of 0-40\% EtOAc/hexanes) and the fractions containing product were combined and concentrated. The concentrate was triturated with hexanes to yield the product as a white solid $(2.5 \mathrm{~g}, 15 \mathrm{mmol}, 38 \%)$. Analytical data: ${ }^{8}{ }^{1} \mathbf{H}$ NMR $\left(400 \mathrm{MHz}, \mathrm{CDCl}_{3}, 298 \mathrm{~K}\right): \delta_{\mathrm{H}} 7.35-7.26(\mathrm{~m}, 2 \mathrm{H}), 6.94-6.87(\mathrm{~m}, 2 \mathrm{H}), 3.83(\mathrm{~s}, 3 \mathrm{H}), 2.41$ (br s, 1H), $1.28-1.19(\mathrm{~m}, 2 \mathrm{H}), 1.05-0.95(\mathrm{~m}, 2 \mathrm{H}) \mathrm{ppm} ;{ }^{13} \mathbf{C} \mathbf{N M R}\left(100 \mathrm{MHz}, \mathrm{CDCl}_{3}, 298 \mathrm{~K}\right): \delta_{\mathrm{C}}$ $158.6,136.3,126.5,113.9,56.8,55.5,16.9 \mathrm{ppm}$. 


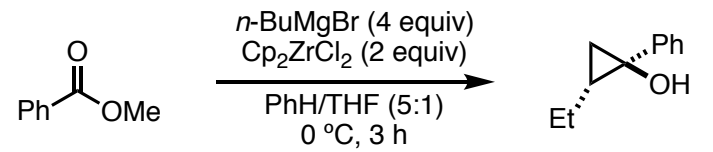

(trans)-2-Ethyl-1-phenylcyclopropan-1-ol (S31): ${ }^{15}$ A flame-dried 100-mL flask with a stir bar was brought into a glovebox and bis(cyclopentadienyl)zirconium(IV) dichloride (0.58 g, 2.0 mmol, 2.0 equiv) was added. The flask was sealed and removed from the glovebox, equipped with a $\mathrm{N}_{2}$ balloon, and $\mathrm{PhH}(31 \mathrm{~mL})$ was added. The solution was cooled to $0{ }^{\circ} \mathrm{C}$.

Butylmagnesium bromide $(6.1 \mathrm{~mL}$ of a $0.66 \mathrm{M}$ solution in THF, $4.0 \mathrm{mmol}, 4.0$ equiv) was added down the side of the flask (total reaction volume $=37 \mathrm{~mL}$ of a 5:1 $\mathrm{PhH} / \mathrm{THF}$ mixture). The reaction was stirred at $0{ }^{\circ} \mathrm{C}$ for $30 \mathrm{~min}$. Then, methyl benzoate $(0.12 \mathrm{~mL}, 1.0 \mathrm{mmol}, 1.0$ equiv) was added dropwise, and the reaction was stirred at $0{ }^{\circ} \mathrm{C}$ for $3 \mathrm{~h}$. The reaction was quenched with $1 \mathrm{M} \mathrm{HCl}$ and extracted with $\mathrm{Et}_{2} \mathrm{O}(\times 3)$, and the organic fractions were combined, washed with brine $(\times 1)$, dried over $\mathrm{MgSO}_{4}$, and concentrated. The crude residue was purified by flash column chromatography (gradient of 0-20\% EtOAc/hexanes) to yield $43 \mathrm{mg}$ of (trans)-2-ethyl-1phenylcyclopropan-1-ol (0.27 mmol, 27\%) and $11 \mathrm{mg}$ of (cis)-2-ethyl-1-phenylcyclopropan-1-ol $(0.068 \mathrm{mmol}, 7 \%)$. The same procedure was repeated on $3.0-\mathrm{mmol}$ scale to yield $0.23 \mathrm{~g}$ of the desired trans product (1.4 mmol, 47\%). The analytical data for (cis)-2-ethyl-1-

phenylcyclopropan-1-ol was identical to the previously prepared sample. The analytical data for (trans)-2-ethyl-1-phenylcyclopropan-1-ol was consistent with literature: ${ }^{15}{ }^{1} \mathbf{H}$ NMR $(500 \mathrm{MHz}$, $\left.\mathrm{CDCl}_{3}, 298 \mathrm{~K}\right): \delta_{\mathrm{H}} 7.45-7.40(\mathrm{~m}, 2 \mathrm{H}), 7.37-7.32(\mathrm{~m}, 2 \mathrm{H}), 7.30-7.25(\mathrm{~m}, 1 \mathrm{H}), 1.45-1.34(\mathrm{~m}$, $1 \mathrm{H}), 1.18-1.06(\mathrm{~m}, 2 \mathrm{H}), 0.92(\mathrm{dd}, J=6.7,5.6 \mathrm{~Hz}, 1 \mathrm{H}), 0.87-0.78(\mathrm{~m}, 4 \mathrm{H}) \mathrm{ppm} ;{ }^{13} \mathbf{C} \mathbf{N M R}(125$ $\left.\mathrm{MHz}, \mathrm{CDCl}_{3}, 298 \mathrm{~K}\right): \delta_{\mathrm{C}} 140.6,128.3,128.2,127.5,62.0,29.3,22.9,18.3,13.5$ ppm; $\mathbf{R}_{\mathbf{f}}(9: 1$ hexanes/EtOAc; $\left.\mathrm{UV} / \mathrm{KMnO}_{4}\right): 0.20$.

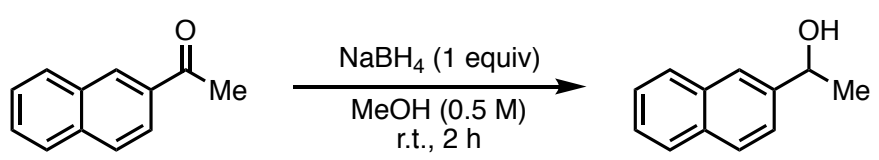

1-(Naphthalen-2-yl)ethan-1-ol (racemic) (S32): To a 100-mL flask with a stir bar were sequentially added 2-naphthyl methyl ketone (1.7 g, $10 \mathrm{mmol}, 1.0$ equiv), MeOH (20 mL, 0.50 $\mathrm{M})$, and sodium borohydride $(0.38 \mathrm{~g}, 10 \mathrm{mmol}, 1.0$ equiv), and the reaction was stirred at $\mathrm{r}$.t. for $2 \mathrm{~h}$. The reaction was quenched with $1 \mathrm{M} \mathrm{HCl}$ and extracted with EtOAc $(\times 3)$. The organic fractions were combined, washed with $\mathrm{H}_{2} \mathrm{O}(\times 1)$ and brine $(\times 1)$, dried over $\mathrm{MgSO}_{4}$, and concentrated to yield the product as a white solid (1.7 g, 9.9 mmol, 99\%). Analytical data: ${ }^{16}{ }^{1} \mathbf{H}$ NMR $\left(400 \mathrm{MHz}, \mathrm{CDCl}_{3}, 298 \mathrm{~K}\right): \delta_{\mathrm{H}} 7.99-7.70(\mathrm{~m}, 4 \mathrm{H}), 7.60-7.36(\mathrm{~m}, 3 \mathrm{H}), 5.07$ (br q, $J=6.9$ $\mathrm{Hz}, 1 \mathrm{H}), 1.99$ (br s, 1H), 1.59 (d, $J=6.5 \mathrm{~Hz}, 3 \mathrm{H}) \mathrm{ppm} ;{ }^{13} \mathbf{C ~ N M R}\left(100 \mathrm{MHz}, \mathrm{CDCl}_{3}, 298 \mathrm{~K}\right): \delta_{\mathrm{C}}$ $143.3,133.5,133.1,128.5,128.1,127.8,126.3,125.9,124.0,123.9,70.7,25.3$ ppm; $\mathbf{R}_{\mathbf{f}}(9: 1$ hexanes/EtOAc; $\left.\mathrm{UV} / \mathrm{KMnO}_{4}\right): 0.17$.

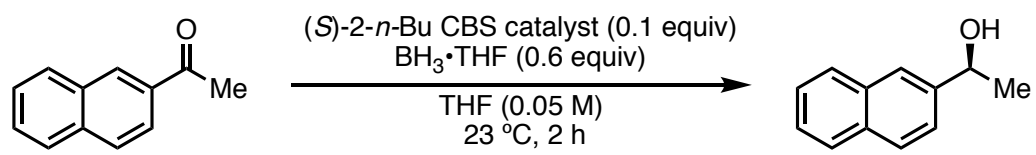

1-(Naphthalen-2-yl)ethan-1-ol (enantioenriched) (S33): To a 100-mL flask was added 2naphthyl methyl ketone $(0.17 \mathrm{~g}, 1.0 \mathrm{mmol}, 1.0$ equiv) and THF $(18 \mathrm{~mL})$, and the solution was submerged in a $23^{\circ} \mathrm{C}$ water bath. $\mathrm{BH}_{3}-\mathrm{THF}(0.60 \mathrm{~mL}$ of a $1.0 \mathrm{~mL}$ solution in THF, $0.60 \mathrm{mmol}$, 0.60 equiv) was added to the reaction, followed by a stock solution of $(S)-(-)-2-$ Butyl-CBS- 
oxazaborolidine catalyst $(0.10 \mathrm{~mL}$ of a $1.0 \mathrm{M}$ solution of catalyst in PhMe diluted with $2.0 \mathrm{~mL}$ THF, $0.10 \mathrm{mmol}, 0.10$ equiv), and the reaction was stirred at $23{ }^{\circ} \mathrm{C}$ for $2 \mathrm{~h}$. The reaction was quenched with $\mathrm{H}_{2} \mathrm{O}$ and extracted with $\mathrm{Et}_{2} \mathrm{O}$, and the organic fractions were combined, washed with brine $(\times 1)$, dried over $\mathrm{MgSO}_{4}$, and concentrated. The crude residue was purified by flash column chromatography (gradient of 0-40\% EtOAc/hexanes) to yield the product as a white solid (71 mg, $0.41 \mathrm{mmol}, 41 \%$ ). The analytical data was identical to that of the racemic product. The e.e. of the material was determined in the next step (compound 6 ). 


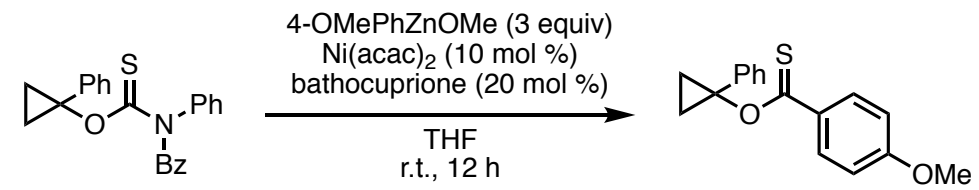

$O$-(1-Phenylcyclopropyl) 4-methoxybenzothioate (4a): The product was prepared according to General Procedure A with the modifications that THF was used instead of 1,4-dioxane and that no magnesium(II) chloride was added. The crude residue was purified by flash column chromatography (gradient of $0-60 \% \mathrm{PhMe} /$ hexanes) to yield the product as a yellow oil which slowly solidified upon standing. The product was prepared on $0.20-\mathrm{mmol}$ scale $(33 \mathrm{mg}$ isolated, $0.116 \mathrm{mmol}, 58 \%)$ and on $1.0-\mathrm{mmol}$ scale $(81 \mathrm{mg}$ isolated, $0.285 \mathrm{mmol}, 29 \%) .{ }^{1} \mathbf{H}$ NMR (500 $\left.\mathrm{MHz} \mathrm{CDCl}_{3}, 298 \mathrm{~K}\right): \delta_{\mathrm{H}} 8.24-8.18(\mathrm{~m}, 2 \mathrm{H}), 7.31-7.27(\mathrm{~m}, 2 \mathrm{H}), 7.27-7.23(\mathrm{~m}, 2 \mathrm{H}), 7.22-7.17$ $(\mathrm{m}, 1 \mathrm{H}), 6.89-6.85(\mathrm{~m}, 2 \mathrm{H}), 3.87(\mathrm{~s}, 3 \mathrm{H}), 1.62-1.56(\mathrm{~m}, 2 \mathrm{H}), 1.49-1.43(\mathrm{~m}, 2 \mathrm{H}) \mathrm{ppm} ;{ }^{13} \mathbf{C}$ NMR $\left(125 \mathrm{MHz}, \mathrm{CDCl}_{3}, 298 \mathrm{~K}\right): \delta_{\mathrm{C}} 208.6,163.8,140.0,131.9,131.1,128.4,126.8,125.2,113.4$, 65.3, 55.7, 17.4 ppm; HRMS $m / z$ (DART): calcd for $\mathrm{C}_{17} \mathrm{H}_{17} \mathrm{O}_{2} \mathrm{~S}(\mathrm{M}+\mathrm{H})$ : 285.0944; found: 285.0951; IR (neat): 3077, 3018, 2974, 2929, 2836, 1727, 1596, 1498, 1453, 1421, 1328, 1277, 1249, 1195, 1169, 1112, 1097, 1023, $837 \mathrm{~cm}^{-1}$; m.p.: 52-55 ${ }^{\circ} \mathrm{C}$; $\mathbf{R}_{\mathbf{f}}(40 \%$ PhMe/hexanes; UV/panisaldehyde): 0.31 .

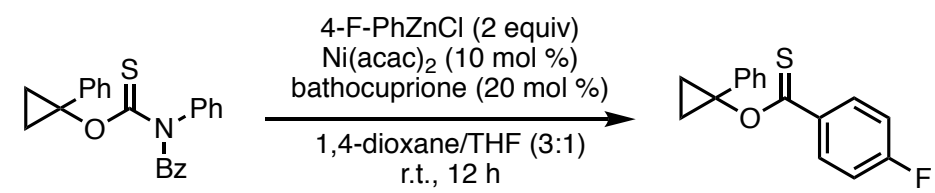

$\boldsymbol{O}$-(1-Phenylcyclopropyl) 4-fluorobenzothioate (4b): The product was prepared according to General Procedure A on 0.20 -mmol scale with the modifications that 4-fluorophenylzinc chloride (2.0 equiv) was used and that no magnesium(II) chloride was added. The crude residue was purified by flash column chromatography (gradient of $0-40 \% \mathrm{PhMe} /$ hexanes) to yield the product as a yellow solid (31 mg, $0.114 \mathrm{mmol}, 57 \%) .{ }^{1} \mathbf{H}$ NMR (500 MHz, $\left.\mathrm{CDCl}_{3}, 298 \mathrm{~K}\right)$ : 8.26$8.21(\mathrm{~m}, 2 \mathrm{H}), 7.33-7.24(\mathrm{~m}, 4 \mathrm{H}), 7.24-7.19(\mathrm{~m}, 1 \mathrm{H}), 7.09-7.03(\mathrm{~m}, 2 \mathrm{H}), 1.61-1.56(\mathrm{~m}, 2 \mathrm{H})$, 1.50-1.45 (m, 2H) ppm; ${ }^{19} \mathbf{F}$ NMR $\left(376 \mathrm{MHz}, \mathrm{CDCl}_{3}, 298 \mathrm{~K}\right): \delta_{\mathrm{F}}-106.3 \mathrm{ppm} ;{ }^{13} \mathbf{C}$ NMR (125 $\left.\mathrm{MHz}, \mathrm{CDCl}_{3}, 298 \mathrm{~K}\right): \delta_{\mathrm{C}} 207.8,166.0(\mathrm{~d}, J=253.7 \mathrm{~Hz}), 139.5,135.1(\mathrm{~d}, J=2.8 \mathrm{~Hz}), 131.3(\mathrm{~d}, J$ $=9.1 \mathrm{~Hz}), 128.4,127.0,125.5,115.3(\mathrm{~d}, J=21.9 \mathrm{~Hz}), 65.9,17.1 \mathrm{ppm}$; HRMS $m / z$ (DART): calcd for $\mathrm{C}_{16} \mathrm{H}_{14} \mathrm{OFS}(\mathrm{M}+\mathrm{H})$ : 273.0744; found: 273.0747; IR (neat): 2924, 2854, 1599, 1503 , 1455, 1412, 1269, 1231, 1196, 1155, 1097, 1041, 843, 830, $696 \mathrm{~cm}^{-1}$; m.p.: $42-44{ }^{\circ} \mathrm{C} ; \mathbf{R}_{\mathbf{f}}(40 \%$ $\mathrm{PhMe} /$ hexanes; UV/p-anisaldehyde) 0.60 . 


\section{F. Mechanistic experiments}

\section{F.1. Barton-McCombie deoxygenation of $\mathbf{1 k}$}

Equation S1. Barton-McCombie deoxygenation of $\mathbf{1 k}$

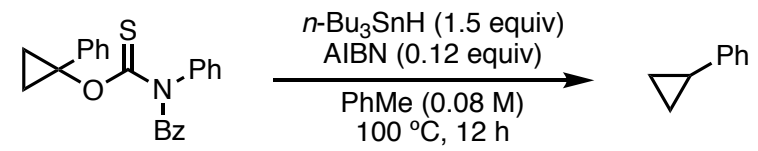

Cyclopropylbenzene (S34): To a 16-mL threaded culture tube with a stir bar was added 1k (75 $\mathrm{mg}, 0.20 \mathrm{mmol}, 1.0$ equiv) and the tube was sealed and evacuated and backfilled with $\mathrm{N}_{2}(\times 3)$. PhMe (degassed, $1.5 \mathrm{~mL}$ ) was added, followed by tributyltin hydride $(81 \mu \mathrm{L}, 0.30 \mathrm{mmol}, 1.5$ equiv), and AIBN (3.9 mg as a stock solution in $1.0 \mathrm{~mL}$ PhMe, $0.024 \mathrm{mmol}, 0.12$ equiv). The reaction was heated at $100{ }^{\circ} \mathrm{C}$ for $12 \mathrm{~h}$. The reaction was cooled to r.t., quenched with $\mathrm{H}_{2} \mathrm{O}$, and extracted with $\mathrm{Et}_{2} \mathrm{O}(\times 3)$. The organic fractions were combined, washed with $1 \mathrm{M}$ aq. TBAF, dried over $\mathrm{MgSO}_{4}$, and concentrated. Dibromomethane ( $14 \mu \mathrm{L}, 0.20 \mathrm{mmol}, 1.0$ equiv) was added as internal ${ }^{1} \mathrm{H}$ NMR standard. The yield was determined to be $65 \%$ by ${ }^{1} \mathrm{H}$ NMR. The spectral data for the cyclopropylbenzene product matched literature data. ${ }^{17}$

\section{F.2. Reaction using $4 a$}

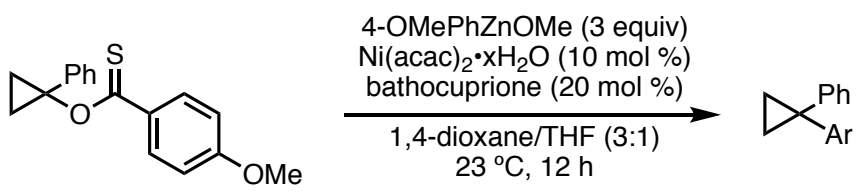

The reaction was performed on 0.10-mmol scale according to General Procedure B. The yield was determined to be $82 \%$ by GC-MS using dodecane as an internal standard. The analytical data was identical to that of $\mathbf{3 a}$ prepared in the standard reaction.

\section{F.3. Crossover experiments}

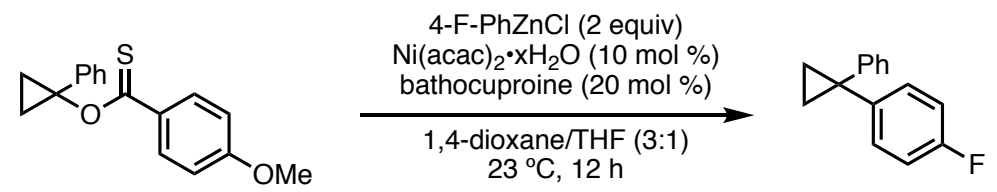

1-Fluoro-4-(1-phenylcyclopropyl)benzene (3b): Prepared according to General Procedure B on 0.10 - $\mathrm{mmol}$ scale from $\mathbf{4 a}(28 \mathrm{mg}$ as a stock solution in $1.3 \mathrm{~mL}$ of 1,4-dioxane, $0.10 \mathrm{mmol}, 1.0$ equiv) and 4-fluorophenylzinc chloride (1.3 mL of a $0.46 \mathrm{M}$ solution in THF, $0.20 \mathrm{mmol}, 2.0$ equiv). The crude residue was purified by flash column chromatography (gradient of $0-10 \%$ $\mathrm{PhMe} /$ hexanes). Cyclopropane $\mathbf{3 b}$ was inseparable from homodimer side-product, 4,4'-difluoro1,1'-biphenyl. A $15 \mathrm{mg}$ sample was obtained which was a 1:1 mixture of $\mathbf{3 b}$ to homodimer as determined by ${ }^{19} \mathrm{~F}$ NMR (desired: $0.049 \mathrm{mmol}, 49 \%$; homodimer: $0.025 \mathrm{mmol}, 25 \%$ ). 4,4'Difluoro-1,1'-biphenyl is a known compound. ${ }^{18}$ Characterization for $\mathbf{3 b}:{ }^{1} \mathbf{H}$ NMR $(400 \mathrm{MHz}$, $\left.\mathrm{CDCl}_{3}, 298 \mathrm{~K}\right): \delta_{\mathrm{H}} 7.30-7.15(\mathrm{~m}, 7 \mathrm{H}), 6.91-6.90(\mathrm{~m}, 2 \mathrm{H}), 1.32-1.28(\mathrm{~m}, 4 \mathrm{H}) \mathrm{ppm} ;{ }^{19} \mathbf{F} \mathbf{N M R}$ 
(376 MHz, $\left.\mathrm{CDCl}_{3}, 298 \mathrm{~K}\right): \delta_{\mathrm{F}}-117.1 \mathrm{ppm} ;{ }^{13} \mathbf{C}$ NMR $\left(125 \mathrm{MHz}, \mathrm{CDCl}_{3}, 298 \mathrm{~K}\right): \delta_{\mathrm{C}} 161.0$ (d, $J$ $=148.0 \mathrm{~Hz}), 145.8,141.5(\mathrm{~d}, J=3.1 \mathrm{~Hz}), 130.3(\mathrm{~d}, J=7.9 \mathrm{~Hz}), 128.5,128.2,126.1,115.1(\mathrm{~d}, J$ $=21.2 \mathrm{~Hz}), 29.9,16.5 \mathrm{ppm} ; \mathbf{R}_{\mathbf{f}}(40 \%$ PhMe/hexanes; UV): 0.58 .
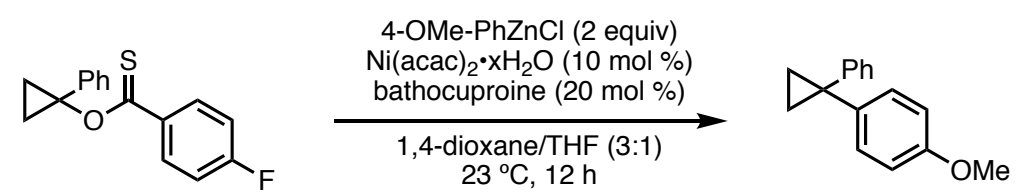

Reaction using O-(1-phenylcyclopropyl) 4-fluorobenzothioate (4b): Performed according to General Procedure B on 0.088 - $\mathrm{mmol}$ scale using $\mathbf{4 b}(24 \mathrm{mg}$ as a solution in $1.7 \mathrm{~mL}$ 1,4-dioxane, $0.088 \mathrm{mmol}, 1.0$ equiv) and 4-methoxyphenylzinc chloride $(0.55 \mathrm{~mL}$ of a $0.33 \mathrm{M}$ solution in THF, $0.18 \mathrm{mmol}, 2.0$ equiv). At the end of the reaction, $n$-dodecane was added as GC-MS standard. The yield of cyclopropane 3a was determined to be 5\% by GC-MS. There was no detectable amount of $\mathbf{3 b}$ by GC-MS. 


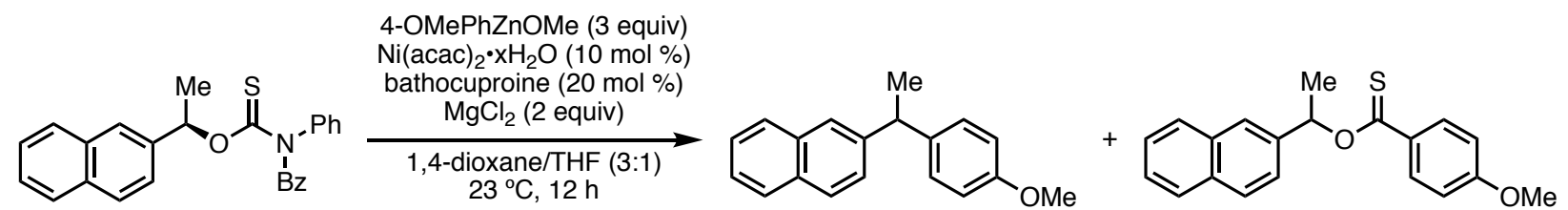

2-(1-(4-Methoxyphenyl)ethyl)naphthalene (16): Performed on 0.10-mmol scale according to General Procedure A using 6 ( $83 \%$ e.e.). The crude residue was purified by flash column chromatography (gradient of $0-20 \% \mathrm{Et}_{2} \mathrm{O} /$ hexanes) to yield 2-(1-(4methoxyphenyl)ethyl)naphthalene (7) $(1.7 \mathrm{mg}, 0.0065 \mathrm{mmol}, 7 \%)$ and thiobenzoate side-product $O$-(1-(naphthalen-2-yl)ethyl) 4-methoxybenzothioate $(3.8 \mathrm{mg}, 0.012 \mathrm{mmol}, 12 \%)$. The reaction was repeated using rac-7 and 4,4'-dimethoxybipyridyl (20 mol \%) to obtain a racemic sample of 8 (8.1 $\mathrm{mg}$ isolated, $0.031 \mathrm{mmol}, 31 \%)$.<smiles>COc1ccc(C(C)c2ccc3ccccc3c2)cc1</smiles>

2-(1-(4-Methoxyphenyl)ethyl)naphthalene (16): White solid. The NMR data was consistent with literature. ${ }^{19}$ Chiral HPLC (Chiralpak IA, $0.2 \% i-\mathrm{PrOH} / \mathrm{hexanes,} 1.0 \mathrm{~mL} / \mathrm{min}, \lambda=227 \mathrm{~nm}$ ): $t_{\mathrm{R}}$ (major): $8.39 \mathrm{~min}$; $t_{\mathrm{R}}$ (minor): $9.45 \mathrm{~min} ; 9 \%$ e.e.<smiles>COc1ccc(C(=S)OC(C)c2ccc3ccccc3c2)cc1</smiles>

$\boldsymbol{O}$-(1-(Naphthalen-2-yl)ethyl) 4-methoxybenzothioate (S35): Yellow solid. ${ }^{\mathbf{1}} \mathbf{H}$ NMR (500 $\left.\mathrm{MHz}, \mathrm{CDCl}_{3}, 298 \mathrm{~K}\right): \delta_{\mathrm{H}} 8.26-8.21(\mathrm{~m}, 2 \mathrm{H}), 7.90-7.81(\mathrm{~m}, 4 \mathrm{H}), 7.57(\mathrm{dd}, J=8.5,1.8 \mathrm{HZ}, 1 \mathrm{H})$, $7.51-7.45(\mathrm{~m}, 2 \mathrm{H}), 6.93(\mathrm{q}, J=6.5 \mathrm{~Hz}, 1 \mathrm{H}), 6.90-6.86(\mathrm{~m}, 2 \mathrm{H}), 3.87$ (s, 3H), 1.84 (d, $J=6.6 \mathrm{~Hz}$, $3 \mathrm{H}) \mathrm{ppm} ;{ }^{13} \mathbf{C}$ NMR $\left(125 \mathrm{MHz}, \mathrm{CDCl}_{3}, 298 \mathrm{~K}\right): \delta_{\mathrm{C}} 209.3,163.8,138.8,133.3,133.2,132.1$, 131.4, 128.6, 128.2, 127.8, 126.4, 126.3, 125.5, 124.4, 113.4, 79.5, 55.7, 22.0 ppm; HRMS m/z (DART): calcd for $\mathrm{C}_{20} \mathrm{H}_{19} \mathrm{O}_{2} \mathrm{~S}(\mathrm{M}+\mathrm{H}): 323.1100$; found: 323.1098; IR (neat): 2971, 2921, 2850, 1597, 1505, 1454, 1316, 1253, 1218, 1169, 1110, 1055, 1024, 839, 828, 747, $639 \mathrm{~cm}^{-1}$; m.p.: 78-80 ${ }^{\circ} \mathrm{C} ; \mathbf{R}_{\mathbf{f}}(9: 1$ hexanes/EtOAc; UV/p-anisaldehyde): 0.47. 

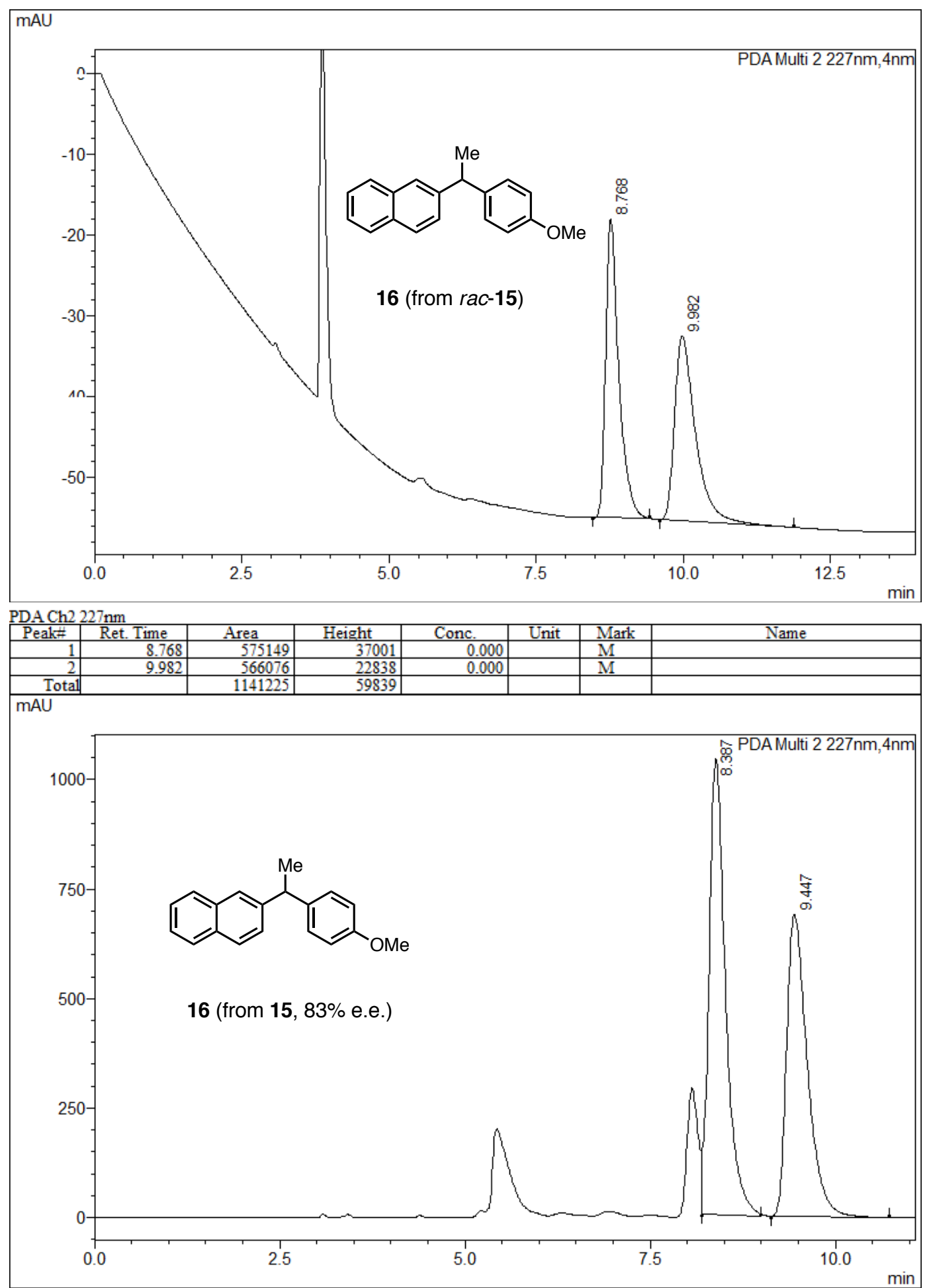

PDA Ch2 227nm
\begin{tabular}{|r|r|r|r|r|r|r|r|}
\hline Peak\# & Ret. Time & Area & Height & Conc. & Unit & Mark & Name \\
\hline 1 & 8.387 & 16136263 & 1041944 & 0.000 & & M & \\
\hline 2 & 9.447 & 13365471 & 689875 & 0.000 & & & \\
\hline Total & & 29501734 & 1731819 & & & & \\
\hline
\end{tabular}




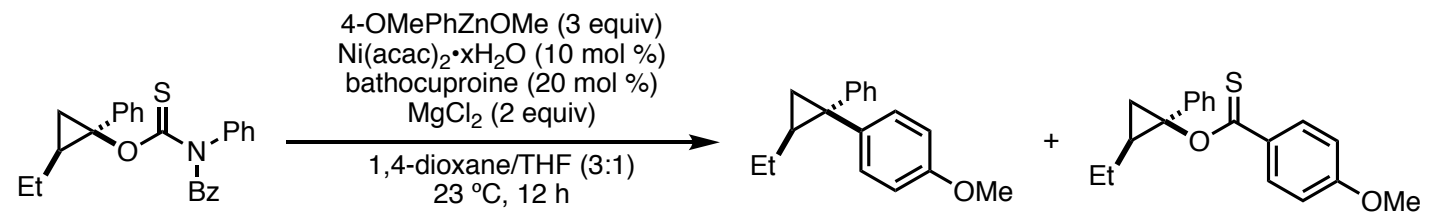

Reaction using cis-17: The reaction was performed on $0.20-\mathrm{mmol}$ scale according to General Procedure A. The crude residue was purified by flash column chromatography (gradient of 0 $40 \% \mathrm{PhMe} /$ hexanes $)$ to yield cyclopropane 1-((cis)-2-ethyl-1-phenylcyclopropyl)-4methoxybenzene (cis-19) (9.1 mg, $0.036 \mathrm{mmol}, 18 \%)$ and thioester side-product $O-((\mathrm{cis})-2-$ ethyl-1-phenylcyclopropyl) 4-methoxybenzothioate (12 mg, $0.038 \mathrm{mmol}, 19 \%$ ). The crude material was analyzed by GC-MS to determine the d.r. of the cyclopropane product to be $>20: 1$ cis/trans, which was confirmed by ${ }^{1} \mathrm{H}$ NMR of the crude material.<smiles>CCC1CC1(c1ccccc1)c1ccc(OC)cc1</smiles>

1-((cis)-2-Ethyl-1-phenylcyclopropyl)-4-methoxybenzene (cis-19): Colourless oil. ${ }^{1} \mathbf{H}$ NMR (500 MHz, $\left.\mathrm{CDCl}_{3}, 298 \mathrm{~K}\right): \delta_{\mathrm{H}} 7.26-7.18(\mathrm{~m}, 4 \mathrm{H}), 7.17-7.13(\mathrm{~m}, 2 \mathrm{H}), 7.12-7.08(\mathrm{~m}, 1 \mathrm{H}), 6.86-$ $6.81(\mathrm{~m}, 2 \mathrm{H}), 3.79(\mathrm{~s}, 3 \mathrm{H}), 1.55-1.49(\mathrm{~m}, 1 \mathrm{H}), 1.46-1.36(\mathrm{~m}, 1 \mathrm{H}), 1.19(\mathrm{ddd}, J=8.7,4.6,0.6$ $\mathrm{Hz}, 1 \mathrm{H}), 1.12(\mathrm{dd}, J=6.0,4.6 \mathrm{~Hz}, 1 \mathrm{H}), 0.98(\mathrm{t}, J=7.4 \mathrm{~Hz}, 3 \mathrm{H}), 0.91-0.79(\mathrm{~m}, 1 \mathrm{H}) \mathrm{ppm} ;{ }^{13} \mathbf{C}$ NMR (125 MHz, $\left.\mathrm{CDCl}_{3}, 298 \mathrm{~K}\right): \delta_{\mathrm{C}} 158.1,148.1,134.2,131.7,128.2,127.6,125.5,113.6,55.3$, 34.8, 28.7, 24.3, 21.0, 13.9 ppm; HRMS $m / z$ (DART): calcd for $\mathrm{C}_{18} \mathrm{H}_{21} \mathrm{O}(\mathrm{M}+\mathrm{H})$ : 253.1587; found: 253.1584; IR (neat): 2957, 2926, 2854, 1609, 1512, 1455, 1246, 1177, 1036, $699 \mathrm{~cm}^{-1}$; $\mathbf{R}_{\mathbf{f}}(40 \%$ PhMe/hexanes; UV/p-anisaldehyde): 0.66 .<smiles>CCC1CC1OC(=S)c1ccc(OC)cc1</smiles>

O-((cis)-2-Ethyl-1-phenylcyclopropyl) 4-methoxybenzothioate (S36): Yellow solid. ${ }^{1}$ H NMR $\left(500 \mathrm{MHz}, \mathrm{CDCl}_{3}, 298 \mathrm{~K}\right): \delta_{\mathrm{H}} 8.25-8.21(\mathrm{~m}, 2 \mathrm{H}), 7.31-7.25(\mathrm{~m}, 2 \mathrm{H}), 7.23-7.14(\mathrm{~m}, 3 \mathrm{H}), 6.91-$ $6.86(\mathrm{~m}, 2 \mathrm{H}), 3.87(\mathrm{~s}, 3 \mathrm{H}), 1.88(\mathrm{dd}, J=9.6,7.1 \mathrm{~Hz}, 1 \mathrm{H}), 1.85-1.75(\mathrm{~m}, 1 \mathrm{H}), 1.68-1.59(\mathrm{~m}, 1 \mathrm{H})$, 1.39-1.30 (m, 1H), 1.20-1.14 (m, 4H) ppm; $\left.{ }^{13} \mathbf{C ~ N M R ~ ( 1 2 5 ~ M H z , ~} \mathrm{CDCl}_{3}, 298 \mathrm{~K}\right): \delta_{\mathrm{C}} 208.8$, 163.8, 141.1, 132.0, 131.0, 128.4, 126.5, 124.9, 113.5, 68.3, 55.7, 32.0, 22.5, 21.9, 14.1 ppm; HRMS $m / z$ (DART) calcd for $\mathrm{C}_{19} \mathrm{H}_{21} \mathrm{O}_{2} \mathrm{~S}(\mathrm{M}+\mathrm{H}): 313.1257$; found: 313.1255 ; IR (neat): 2960, 2927, 2839, 1596, 1573, 1502, 1452, 1330, 1277, 1253, 1204, 1161, 1111, 1020, 838, 742, 695 cm ${ }^{-1}$; m.p.: $82-92{ }^{\circ} \mathrm{C}$; $\mathbf{R}_{\mathbf{f}}(40 \%$ PhMe/hexanes; UV/p-anisaldehyde): 0.59 . 


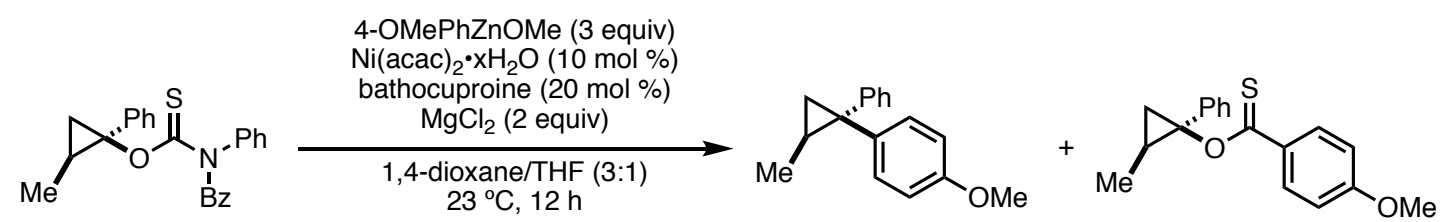

Reaction using cis-18: The reaction was performed on $0.20-\mathrm{mmol}$ scale according to General Procedure A. The crude residue was purified by flash column chromatography (gradient of 0 40\% PhMe/hexanes) to yield cyclopropane 1-methoxy-4-((cis)-2-methyl-1-

phenylcyclopropyl)benzene (cis-20) $(9.3 \mathrm{mg}, 0.039 \mathrm{mmol}, 20 \%)$ and thioester side-product $O$ ((cis)-2-methyl-1-phenylcyclopropyl) 4-methoxybenzothioate (38 mg, $0.127 \mathrm{mmol}, 64 \%$ ). The crude material was analyzed by GC-MS to determine the d.r. of the cyclopropane product to be $>20: 1$ cis/trans, which was confirmed by ${ }^{1} \mathrm{H}$ NMR of the crude material.
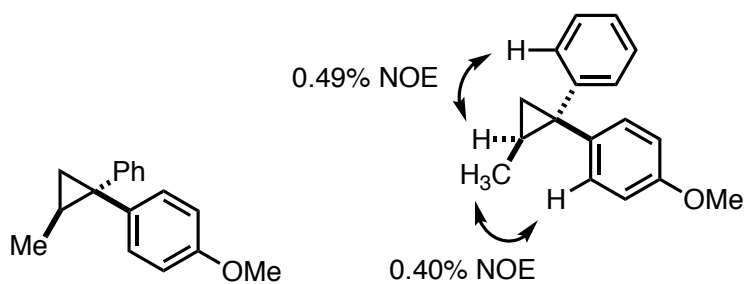

1-Methoxy-4-((cis)-2-methyl-1-phenylcyclopropyl)benzene (cis-20): Colourless oil. The relative configuration was determined by NOE analysis. ${ }^{1} \mathbf{H} \mathbf{N M R}\left(500 \mathrm{MHz}, \mathrm{CDCl}_{3}, 298 \mathrm{~K}\right): \delta_{\mathrm{H}}$ 7.26-7.17 (m, 4H), 7.14-7.07 (m, 3H), 6.88-6.83 (m, 2H), 3.80 (s, 3H), 1.66-1.57 (m, 1H), 1.25 (ddd, $J=8.7,4.6,1.0 \mathrm{~Hz}, 1 \mathrm{H}), 1.08$ (ddd, $J=5.7,4.5,1.0 \mathrm{~Hz}, 1 \mathrm{H}), 0.94(\mathrm{~d}, J=6.2 \mathrm{~Hz}, 3 \mathrm{H}) \mathrm{ppm}$; ${ }^{13} \mathrm{C}$ NMR (125 MHz, $\left.\mathrm{CDCl}_{3}, 298 \mathrm{~K}\right): \delta_{\mathrm{C}} 158.1,148.2,133.9,132.0,128.2,127.3,125.5,113.7$, 55.3, 34.3, 22.9, 21.0, 16.4 ppm; HRMS $m / z$ (DART): calcd for $\mathrm{C}_{17} \mathrm{H}_{22} \mathrm{NO}\left(\mathrm{M}+\mathrm{NH}_{4}\right)$ : 256.1696; found: 256.1699; IR (neat): 3061, 3000, 2946, 2835, 1608, 1511, 1496, 1443, 1290, 1242, 1176, 1032, 822, 745, $697 \mathrm{~cm}^{-1}$; $\mathbf{R}_{\mathbf{f}}(40 \%$ PhMe/hexanes; UV/p-anisaldehyde): 0.64.<smiles>COc1ccc(C(=S)OC2CC2C)cc1</smiles>

O-((cis)-2-Methyl-1-phenylcyclopropyl) 4-methoxybenzothioate (S37): Yellow oil which slowly solidified upon standing. ${ }^{1} \mathbf{H}$ NMR $\left(500 \mathrm{MHz}, \mathrm{CDCl}_{3}, 298 \mathrm{~K}\right): \delta_{\mathrm{H}} 8.28-8.23(\mathrm{~m}, 2 \mathrm{H})$, 7.30-7.25 (m, 2H), 7.21-7.15 (m, 3H), 6.91-6.87 (m, 2H), $3.88(\mathrm{~s}, 3 \mathrm{H}), 1.80(\mathrm{dd}, J=9.6,7.0$ $\mathrm{Hz}, 1 \mathrm{H}), 1.56-1.47(\mathrm{~m}, 1 \mathrm{H}), 1.39(\mathrm{~d}, J=6.2 \mathrm{~Hz}, 3 \mathrm{H}), 1.16(\mathrm{dd}, J=7.7,7.0 \mathrm{~Hz}, 1 \mathrm{H}) \mathrm{ppm} ;{ }^{13} \mathbf{C}$ NMR $\left(125 \mathrm{MHz}, \mathrm{CDCl}_{3}, 298 \mathrm{~K}\right): \delta_{\mathrm{C}} 208.9,163.8,141.1,132.0,131.1,128.3,126.4,124.7$, 113.5, 68.1, 55.7, 24.3, 23.3, 13.8 ppm; HRMS $m / z$ (DART): calcd for $\mathrm{C}_{18} \mathrm{H}_{19} \mathrm{O}_{2} \mathrm{~S}(\mathrm{M}+\mathrm{H})$ : 299.1100; found: 299.1093; IR (neat): 3005, 2960, 2929, 2839, 1592, 1500, 1450, 1330, 1278, 1250, 1171, 1110, 1021, 834, 749, $694 \mathrm{~cm}^{-1}$; m.p.: $99-101^{\circ} \mathrm{C} ; \mathbf{R}_{\mathbf{f}}(40 \%$ PhMe/hexanes; UV/panisaldehyde): 0.58 . 


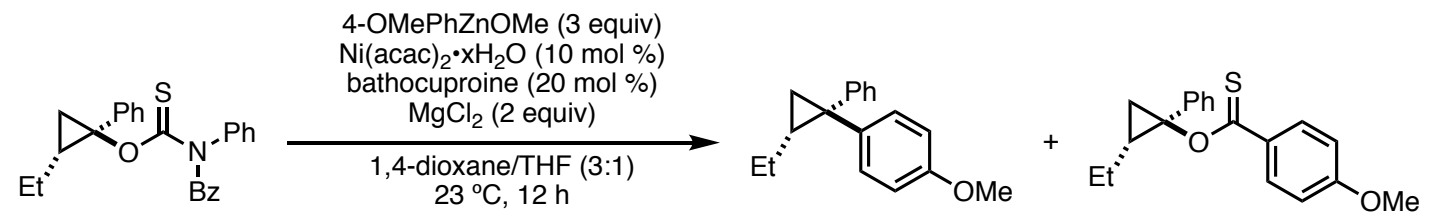

Reaction using trans-17: The reaction was performed on $0.10-\mathrm{mmol}$ scale according to General Procedure A. The crude residue was purified by flash column chromatography (gradient of 0 $30 \% \mathrm{PhMe} /$ hexanes $)$ to yield cyclopropane product 1-((trans)-2-ethyl-1-phenylcyclopropyl)-4methoxybenzene (trans-19) (3.5 mg, $0.014 \mathrm{mmol}, 14 \%)$ and thioester side-product $O$ - $(($ trans $)-2$ ethyl-1-phenylcyclopropyl) 4-methoxybenzothioate (17 mg, $0.054 \mathrm{mmol}, 54 \%$ ). The crude material was analyzed by GC-MS to determine the d.r. of the cyclopropane product to be $>20: 1$ trans/cis, which was confirmed by ${ }^{1} \mathrm{H}$ NMR of the crude material.

The reaction was repeated on $0.40-\mathrm{mmol}$ scale using 4.0 equiv of arylzinc reagent, $20 \mathrm{~mol} \%$ $\mathrm{Ni}(\mathrm{acac})_{2} \bullet \mathrm{xH}_{2} \mathrm{O}$, and $40 \mathrm{~mol} \%$ bathocuproine to yield $28 \mathrm{mg}$ of trans $\mathbf{1 9}(0.111 \mathrm{mmol}, 28 \%)$ and $18 \mathrm{mg}$ of thioester side-product $O$-((trans)-2-ethyl-1-phenylcyclopropyl) 4methoxybenzothioate $(0.058 \mathrm{mmol}, 15 \%)$. The d.r. of the crude material was $>20: 1$.<smiles>CCC1CC1c1ccc(OC)cc1</smiles>

1-((trans)-2-Ethyl-1-phenylcyclopropyl)-4-methoxybenzene (trans-19): Colourless oil. ${ }^{\mathbf{1}} \mathbf{H}$ NMR $\left(400 \mathrm{MHz}, \mathrm{CDCl}_{3}, 298 \mathrm{~K}\right): \delta_{\mathrm{H}} 7.35-7, .24(\mathrm{~m}, 4 \mathrm{H}), 7.22-7.11(\mathrm{~m}, 3 \mathrm{H}), 6.81-6.74(\mathrm{~m}, 2 \mathrm{H})$, $3.76(\mathrm{~s}, 3 \mathrm{H}), 1.57-1.50(\mathrm{~m}, 1 \mathrm{H}), 1.45-1.33(\mathrm{~m}, 1 \mathrm{H}), 1.18-1.12(\mathrm{~m}, 2 \mathrm{H}), 0.98(\mathrm{t}, J=7.3 \mathrm{~Hz}, 3 \mathrm{H})$, 0.93-0.77 (m, 1H) ppm; ${ }^{13} \mathbf{C}$ NMR (100 MHz, $\left.\mathrm{CDCl}_{3}, 298 \mathrm{~K}\right): 157.7,142.6,140.1,130.5,129.1$, 128.2 , 126.1, 113.7, 55.4, 35.1, 28.1, 24.2, 20.1, 13.9 ppm; HRMS $\mathrm{m} / z$ (DART): calcd for $\mathrm{C}_{18} \mathrm{H}_{21} \mathrm{O}(\mathrm{M}+\mathrm{H}): 253.1587$; found: 253.1590; IR (neat): 3063, 2999, 2958, 2872, 2834, 1613, 1496, 1462, 1446, 1288, 1243, 1178, 806, 828, 785, $699 \mathrm{~cm}^{-1}$; $\mathbf{R}_{\mathbf{f}}(9: 1$ hexanes/EtOAc; UV): 0.50 .<smiles>CC[C@H]1C[C@@H]1OC(=S)c1ccc(OC)cc1</smiles>

O-((trans)-2-Ethyl-1-phenylcyclopropyl) 4-methoxybenzothioate (S38): Yellow solid. ${ }^{\mathbf{1}} \mathbf{H}$ NMR (500 MHz, $\left.\mathrm{CDCl}_{3}, 298 \mathrm{~K}\right): \delta_{\mathrm{H}} 8.18-8.13(\mathrm{~m}, 2 \mathrm{H}), 7.48-7.43(\mathrm{~m}, 2 \mathrm{H}), 7.33-7.27$ (m, 2H), 7.25-7.20 (m, 1H), 7.87-8.81 (m, 2H), $3.85(\mathrm{~s}, 3 \mathrm{H}), 1.75-1.67(\mathrm{~m}, 1 \mathrm{H}), 1.55-1.46(\mathrm{~m}, 2 \mathrm{H})$, $1.30-1.21(\mathrm{~m}, 1 \mathrm{H}), 1.02-0.92(\mathrm{~m}, 1 \mathrm{H}), 0.90(\mathrm{t}, J=6.9 \mathrm{~Hz}, 3 \mathrm{H}) \mathrm{ppm} ;{ }^{13} \mathbf{C} \mathbf{N M R}(125 \mathrm{MHz}$, $\left.\mathrm{CDCl}_{3}, 298 \mathrm{~K}\right): \delta_{\mathrm{C}} 208.4,163.6,136.7,132.2,131.0,128.9,127.9,127.4,113.3,70.0,55.6,28.4$, 22.3, 18.5, 13.2 ppm; HRMS $m / z$ (DART): calcd for $\mathrm{C}_{19} \mathrm{H}_{21} \mathrm{O}_{2} \mathrm{~S}: 313.1257$; found: 313.1261 ; IR (neat): 2961, 2931, 2870, 1596, 1574, 1506, 1450, 1422, 1275, 1256, 1162, 1112, 1016, 837, $747,698 \mathrm{~cm}^{-1}$; m.p.: $87-89^{\circ} \mathrm{C}$. 
Table S10. Reactions with radical traps

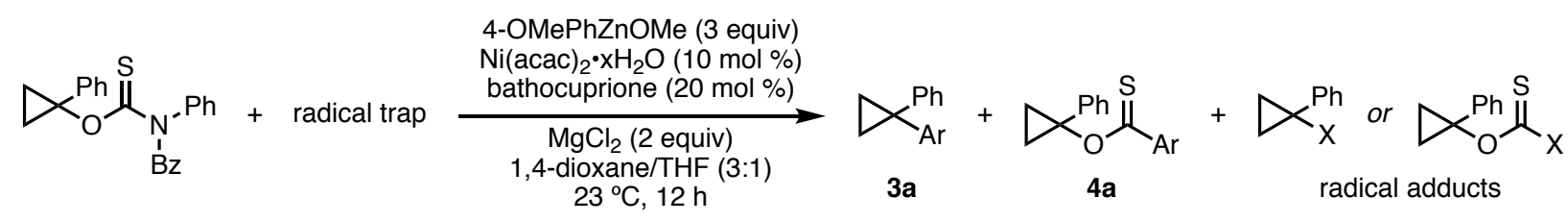

\begin{tabular}{ccccc}
\hline Entry & radical trap (equiv) & Yield 3a (\%) & Yield 4a (\%) & Yield adduct \\
\hline 1 & none & 73 & 12 & N/A \\
2 & TEMPO (1) & 0 & 0 & n.d. \\
3 & styrene (1) & 63 & 13 & n.d. \\
4 & benzyl acrylate (1) & 51 & 14 & n.d. \\
\hline
\end{tabular}

GC-MS yields using $n$-dodecane as internal standard.

Table S11. Yield of cyclopropane and thioester with other leaving groups

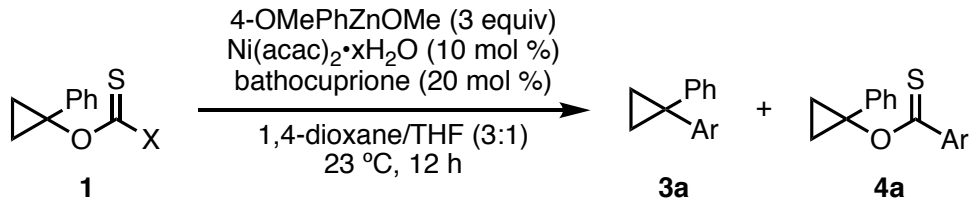

\begin{tabular}{cccc}
\hline Entry & X & Yield 3a (\%) & Yield 4a (\%) \\
\hline 1 & NBzPh & 78 & 12 \\
2 & 1-imidazolyl & 54 & 4 \\
3 & SMe & 29 & 67 \\
\hline
\end{tabular}

GC-MS yields using $n$-dodecane as internal standard. 


\section{G. Computational Details}

All DFT calculations were carried out with the Gaussian 16 software package. ${ }^{20}$ Initial geometries were generated with openbabel $^{21}$ from their SMILES strings, and pre-optimized with Grimme's $x t b$ 6.2.3. ${ }^{22}$ The initial $x t b$-optimized geometries were used for a thorough conformer search with Grimme's crest. $^{23}$ Further geometry optimizations were performed with $\left(\mathrm{SMD}^{24}=1,4\right.$-dioxane) for solvation corrections and the unrestricted $\omega \mathrm{B} 97 \mathrm{X}$ DFT functional ${ }^{25}$ (with an integration grid of pruned 175,974 for first-row atoms and 250,974 for atoms in the second and later rows) with the $6-311++\mathrm{G}(2 \mathrm{~d}, \mathrm{p})$ basis set for all atoms. Grimme's D2 version for empirical dispersion ${ }^{26}$ was also included. Frequency calculations were performed to confirm if a structure is a ground or transition state. Paton's GoodVibes ${ }^{27}$ was used for quasi-harmonic corrections to Gibbs Free Energies (via quasi-harmonic corrections to both entropy and enthalpy, defaulting to the Grimme method for entropy and the Head-Gordon enthalpy correction). Intermediates with their highest Boltzmann weights and their respective transition states were selected for the barriers herein reported. For those structures, Natural Bond Orbital ${ }^{28}$ (NBO) analysis were performed with NBO6 linked to Gaussian 16. NBO analyses were used to gauge the magnitude of the hyperconjugative interactions in the presented systems.

NBO analysis transforms the canonical delocalized molecular orbitals from DFT calculations into localized orbitals that are closely tied to the chemical bonding concepts. Each of the localized NBO sets is complete and orthonormal. The filled NBOs describe the hypothetical, strictly localized Lewis structure. The interactions between filled and antibonding orbitals represent the deviation from the Lewis structure and can be used to measure delocalization. For example, delocalizing interaction can be treated via the $2^{\text {nd }}$ order perturbation energy approach as $E(2)=n_{i}\left|F_{i j}\right|^{2} / \Delta E$, where $\mathrm{n}_{\mathrm{i}}$ is the population of a donor orbitals, $\mathrm{F}_{\mathrm{ij}}$ is the Fock matrix element for the interacting orbitals $\mathrm{i}$ and $\mathrm{j}$, and $\Delta E$ is the energy gap between these orbitals. CYLView ${ }^{29}$ was used to render the molecules. Structures and energies for this SI were prepared with ESIgen. ${ }^{30}$

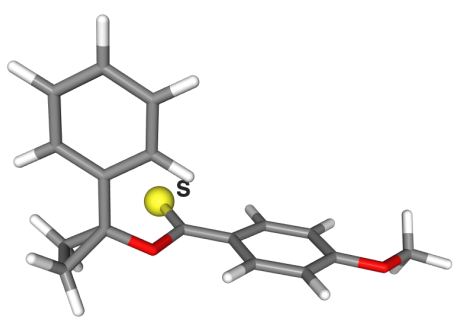

Charge -1

Multiplicity 2

Stoichiometry C17H16O2S(-1)

Electronic Energy (Eh) -1206.0701116

Number of Imaginary Frequencies 0

Mean of alpha and beta Electrons $\quad 75.5$

Molecular Geometry in Cartesian Coordinates

\begin{tabular}{llcc}
\hline $\mathrm{C}$ & 1.705517 & 0.040366 & -1.160821 \\
$\mathrm{C}$ & 2.971935 & -0.450923 & -1.390762 \\
$\mathrm{C}$ & 3.949416 & -0.409355 & -0.395727 \\
$\mathrm{C}$ & 3.622830 & 0.139937 & 0.842725 \\
$\mathrm{C}$ & 2.350834 & 0.633306 & 1.078605 \\
$\mathrm{C}$ & 1.334819 & 0.601230 & 0.093699 \\
$\mathrm{C}$ & 0.023328 & 1.091857 & 0.348180 \\
$\mathrm{~S}$ & -0.547241 & 1.725061 & 1.828720 \\
$\mathrm{O}$ & -0.760262 & 1.058666 & -0.811440 \\
$\mathrm{C}$ & -2.143979 & 0.959381 & -0.719613 \\
$\mathrm{C}$ & -2.667778 & -0.355838 & -0.232899
\end{tabular}




\begin{tabular}{|c|c|c|c|}
\hline C & -1.805814 & -1.442255 & -0.096813 \\
\hline $\mathrm{C}$ & -2.281947 & -2.680357 & 0.309341 \\
\hline $\mathrm{C}$ & -3.629264 & -2.862219 & 0.583911 \\
\hline $\mathrm{C}$ & -4.497043 & -1.787551 & 0.448964 \\
\hline $\mathrm{C}$ & -4.020227 & -0.549214 & 0.047469 \\
\hline $\mathrm{H}$ & -4.719117 & 0.274597 & -0.043589 \\
\hline $\mathrm{H}$ & -5.553114 & -1.910136 & 0.663972 \\
\hline $\mathrm{H}$ & -3.999406 & -3.829370 & 0.905126 \\
\hline $\mathrm{H}$ & -1.588357 & -3.507070 & 0.416136 \\
\hline $\mathrm{H}$ & -0.751026 & -1.315077 & -0.302903 \\
\hline $\mathrm{C}$ & -2.849362 & 1.615045 & -1.883786 \\
\hline $\mathrm{C}$ & -2.903724 & 2.235704 & -0.515964 \\
\hline $\mathrm{H}$ & -3.825873 & 2.208409 & 0.050616 \\
\hline $\mathrm{H}$ & -2.286676 & 3.103841 & -0.334272 \\
\hline $\mathrm{H}$ & -3.731313 & 1.116975 & -2.268546 \\
\hline $\mathrm{H}$ & -2.214084 & 2.079835 & -2.627702 \\
\hline $\mathrm{H}$ & 2.110473 & 1.055032 & 2.046226 \\
\hline $\mathrm{H}$ & 4.354151 & 0.192429 & 1.640008 \\
\hline $\mathrm{O}$ & 5.179189 & -0.924548 & -0.726369 \\
\hline $\mathrm{C}$ & 6.178498 & -0.882410 & 0.257848 \\
\hline $\mathrm{H}$ & 7.069083 & -1.327555 & -0.186808 \\
\hline $\mathrm{H}$ & 6.410722 & 0.145889 & 0.560524 \\
\hline $\mathrm{H}$ & 5.900905 & -1.460946 & 1.147349 \\
\hline $\mathrm{H}$ & 3.228526 & -0.877249 & -2.355108 \\
\hline $\mathrm{H}$ & 0.971319 & -0.003756 & -1.954220 \\
\hline
\end{tabular}

Frequencies (Top 10 out of 102)

1. $\quad 34.3003 \mathrm{~cm}-1$ (Symmetry: A)

2. $\quad 58.9754 \mathrm{~cm}-1$ (Symmetry: A)

3. $\quad 70.1488 \mathrm{~cm}-1$ (Symmetry: A)

4. $\quad 77.8943 \mathrm{~cm}-1$ (Symmetry: A)

5. $\quad 101.4368 \mathrm{~cm}-1$ (Symmetry: A)

6. $\quad 116.9462 \mathrm{~cm}-1$ (Symmetry: A)

7. $\quad 130.3683 \mathrm{~cm}-1$ (Symmetry: A)

8. $\quad 160.7246 \mathrm{~cm}-1$ (Symmetry: A)

9. $\quad 201.1257 \mathrm{~cm}-1$ (Symmetry: A)

10. $211.0946 \mathrm{~cm}-1$ (Symmetry: A)

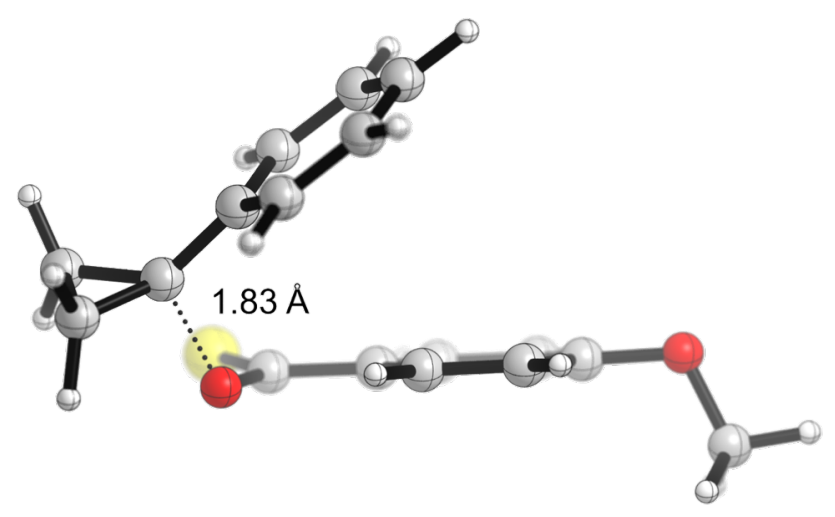

Charge -1

Multiplicity 2

Stoichiometry C17H16O2S(-1)

Electronic Energy (Eh) -1206.03945823 
Number of Imaginary Frequencies 1

Mean of alpha and beta Electrons $\quad 75.5$

Molecular Geometry in Cartesian Coordinates

\begin{tabular}{lrrr}
\hline C & -1.011543 & -0.001316 & -0.931645 \\
C & -2.265544 & 0.581054 & -0.881135 \\
C & -3.241340 & 0.051644 & -0.047896 \\
C & -2.951335 & -1.057001 & 0.734542 \\
C & -1.690357 & -1.628622 & 0.688549 \\
C & -0.689178 & -1.115081 & -0.144643 \\
C & 0.674076 & -1.697286 & -0.215569 \\
S & 1.190910 & -2.926878 & 0.869039 \\
O & 1.444976 & -1.203978 & -1.120618 \\
C & 2.712591 & 0.001212 & -0.577390 \\
C & 2.051096 & 1.104782 & 0.052763 \\
C & 1.771269 & 2.291306 & -0.646318 \\
C & 1.006049 & 3.286694 & -0.070318 \\
$\mathrm{C}$ & 0.486787 & 3.121114 & 1.212918 \\
$\mathrm{C}$ & 0.764554 & 1.957420 & 1.918957 \\
$\mathrm{C}$ & 1.540656 & 0.959502 & 1.352968 \\
$\mathrm{H}$ & 1.738271 & 0.040591 & 1.893720 \\
$\mathrm{H}$ & 0.357556 & 1.818502 & 2.914574 \\
$\mathrm{H}$ & -0.130317 & 3.894743 & 1.655709 \\
$\mathrm{H}$ & 0.797384 & 4.195678 & -0.624852 \\
$\mathrm{H}$ & 2.151513 & 2.409508 & -1.655819 \\
$\mathrm{C}$ & 3.792528 & -0.047412 & -1.569833 \\
$\mathrm{C}$ & 3.919418 & -0.752780 & -0.219741 \\
$\mathrm{H}$ & 4.585866 & -0.322445 & 0.522440 \\
$\mathrm{H}$ & 3.848242 & -1.833683 & -0.210388 \\
$\mathrm{H}$ & 4.381341 & 0.848371 & -1.744747 \\
$\mathrm{H}$ & 3.639636 & -0.677382 & -2.440219 \\
$\mathrm{H}$ & -1.454792 & -2.483982 & 1.309278 \\
$\mathrm{H}$ & -3.717680 & -1.459599 & 1.388579 \\
$\mathrm{H}$ & -2.493127 & 1.457346 & -1.478691 \\
$\mathrm{H}$ & -0.249938 & 0.413594 & -1.578005 \\
$\mathrm{O}$ & -4.487112 & 0.642311 & 0.021773 \\
$\mathrm{C}$ & -5.403891 & 0.119687 & -0.919230 \\
$\mathrm{H}$ & -6.345247 & 0.654130 & -0.782095 \\
$\mathrm{H}$ & -5.050115 & 0.270583 & -1.946073 \\
$\mathrm{H}$ & -5.571087 & -0.952069 & -0.759137
\end{tabular}

Frequencies (Top 10 out of 102)

1. $-782.4990 \mathrm{~cm}-1$ (Symmetry: A) *

2. $24.2976 \mathrm{~cm}-1$ (Symmetry: A)

3. $\quad 30.3713 \mathrm{~cm}-1$ (Symmetry: A)

4. $\quad 50.0140 \mathrm{~cm}-1$ (Symmetry: A)

5. $\quad 70.5248 \mathrm{~cm}-1$ (Symmetry: A)

6. $\quad 75.8368 \mathrm{~cm}-1$ (Symmetry: A)

7. $\quad 95.2858 \mathrm{~cm}-1$ (Symmetry: A)

8. $\quad 98.0492 \mathrm{~cm}-1$ (Symmetry: A)

9. $\quad 145.0029 \mathrm{~cm}-1$ (Symmetry: A)

10. $\quad 163.1225 \mathrm{~cm}-1$ (Symmetry: A) 


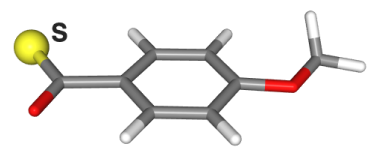

Charge -1

Multiplicity 1

Stoichiometry C8H7O2S(-1)

Electronic Energy (Eh) -857.798235944

Number of Imaginary Frequencies 0

Mean of alpha and beta Electrons 44

Molecular Geometry in Cartesian Coordinates

\begin{tabular}{lrrr}
\hline $\mathrm{C}$ & 0.076357 & 1.455565 & 0.000004 \\
$\mathrm{C}$ & -1.304405 & 1.452348 & 0.000058 \\
$\mathrm{C}$ & -1.998247 & 0.243733 & 0.000047 \\
$\mathrm{C}$ & -1.289569 & -0.952330 & 0.000001 \\
$\mathrm{C}$ & 0.100885 & -0.926189 & -0.000030 \\
$\mathrm{C}$ & 0.808944 & 0.266641 & -0.000022 \\
$\mathrm{C}$ & 2.330551 & 0.345641 & -0.000034 \\
$\mathrm{~S}$ & 3.237904 & -1.128751 & 0.000048 \\
$\mathrm{O}$ & 2.821458 & 1.471859 & -0.000091 \\
$\mathrm{H}$ & 0.660915 & -1.853468 & -0.000049 \\
$\mathrm{H}$ & -1.798712 & -1.907165 & -0.000010 \\
$\mathrm{O}$ & -3.356833 & 0.333892 & 0.000083 \\
$\mathrm{C}$ & -4.093836 & -0.866314 & -0.000084 \\
$\mathrm{H}$ & -5.144628 & -0.577965 & -0.000093 \\
$\mathrm{H}$ & -3.889333 & -1.466260 & -0.893543 \\
$\mathrm{H}$ & -3.889409 & -1.466472 & 0.893250 \\
$\mathrm{H}$ & -1.867354 & 2.378937 & 0.000093 \\
$\mathrm{H}$ & 0.620974 & 2.391840 & -0.000002
\end{tabular}

Frequencies (Top 10 out of 48)

1. $\quad 78.4831 \mathrm{~cm}-1$ (Symmetry: A)

2. $\quad 99.2623 \mathrm{~cm}-1$ (Symmetry: A)

3. $\quad 137.5786 \mathrm{~cm}-1$ (Symmetry: A)

4. $\quad 163.4692 \mathrm{~cm}-1$ (Symmetry: A)

5. $246.2173 \mathrm{~cm}-1$ (Symmetry: A)

6. $280.8262 \mathrm{~cm}-1$ (Symmetry: A)

7. $297.8859 \mathrm{~cm}-1$ (Symmetry: A)

8. $\quad 311.4702 \mathrm{~cm}-1$ (Symmetry: A)

9. $\quad 416.6012 \mathrm{~cm}-1$ (Symmetry: A)

10. $442.7687 \mathrm{~cm}-1$ (Symmetry: A)

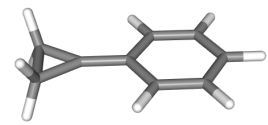

Charge 0

Multiplicity 2

Stoichiometry C9H9

Electronic Energy (Eh) -348.26598436

Number of Imaginary Frequencies 0

Mean of alpha and beta Electrons $\quad 31.5$

Molecular Geometry in Cartesian Coordinates

\begin{tabular}{llll}
\hline C & 1.533260 & 0.000003 & -0.000049
\end{tabular}




$\begin{array}{lrrr}\text { C } & 0.142958 & 0.000002 & -0.000022 \\ \text { C } & -0.588349 & -1.214638 & -0.000009 \\ \text { C } & -1.966880 & -1.205614 & 0.000003 \\ \text { C } & -2.667872 & -0.000001 & 0.000012 \\ \text { C } & -1.966883 & 1.205612 & 0.000003 \\ \text { C } & -0.588352 & 1.214640 & -0.000009 \\ \text { H } & -0.044323 & 2.152506 & -0.000018 \\ \text { H } & -2.509826 & 2.144093 & 0.000013 \\ \text { H } & -3.751329 & -0.000003 & 0.000029 \\ \text { H } & -2.509821 & -2.144096 & 0.000013 \\ \text { H } & -0.044318 & -2.152503 & -0.000019 \\ \text { C } & 2.764221 & -0.770782 & 0.000022 \\ \text { C } & 2.764226 & 0.770780 & 0.000021 \\ \text { H } & 3.075503 & 1.273568 & -0.912578 \\ \text { H } & 3.075326 & 1.273626 & 0.912649 \\ \text { H } & 3.075496 & -1.273572 & -0.912576 \\ \text { H } & 3.075318 & -1.273628 & 0.912651\end{array}$

Frequencies (Top 10 out of 48 )

1. $\quad 48.8021 \mathrm{~cm}-1$ (Symmetry: A)

2. $\quad 137.4509 \mathrm{~cm}-1$ (Symmetry: A)

3. $\quad 140.1076 \mathrm{~cm}-1$ (Symmetry: A)

4. $206.9422 \mathrm{~cm}-1$ (Symmetry: A)

5. $392.4851 \mathrm{~cm}-1$ (Symmetry: A)

6. $\quad 416.8840 \mathrm{~cm}-1$ (Symmetry: A)

7. $\quad 483.0980 \mathrm{~cm}-1$ (Symmetry: A)

8. $484.9088 \mathrm{~cm}-1$ (Symmetry: A)

9. $\quad 629.9552 \mathrm{~cm}-1$ (Symmetry: A)

10. $694.3463 \mathrm{~cm}-1$ (Symmetry: A)

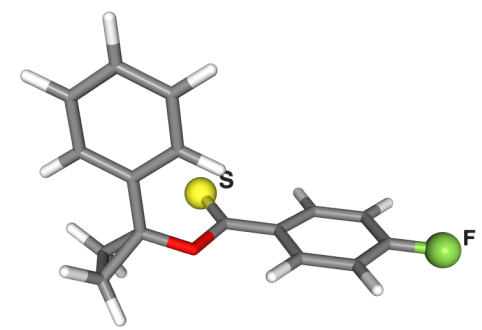

Charge -1

Multiplicity 2

Stoichiometry C16H13FOS(-1)

Electronic Energy (Eh) -1190.7957172

Number of Imaginary Frequencies 0

Mean of alpha and beta Electrons $\quad 71.5$

Molecular Geometry in Cartesian Coordinates

\begin{tabular}{llll}
\hline $\mathrm{C}$ & 2.113758 & -0.105316 & -1.112571 \\
$\mathrm{C}$ & 3.368299 & -0.645405 & -1.328613 \\
$\mathrm{C}$ & 4.280090 & -0.671925 & -0.290195 \\
$\mathrm{C}$ & 3.965820 & -0.171467 & 0.963261 \\
$\mathrm{C}$ & 2.714704 & 0.365806 & 1.181873 \\
$\mathrm{C}$ & 1.734410 & 0.422765 & 0.153672 \\
$\mathrm{C}$ & 0.443075 & 0.971467 & 0.384041 \\
$\mathrm{~S}$ & -0.145580 & 1.585922 & 1.860654 \\
$\mathrm{O}$ & -0.307581 & 1.000430 & -0.793879 \\
$\mathrm{C}$ & -1.697142 & 1.014852 & -0.733736
\end{tabular}




$\begin{array}{lrrr}\text { C } & -2.331915 & -0.256315 & -0.263258 \\ \text { C } & -1.560808 & -1.407482 & -0.114721 \\ \text { C } & -2.140919 & -2.605147 & 0.276708 \\ \text { C } & -3.503646 & -2.681106 & 0.523571 \\ \text { C } & -4.281431 & -1.541326 & 0.375449 \\ \text { C } & -3.700828 & -0.343294 & -0.011628 \\ \text { H } & -4.330731 & 0.533262 & -0.113001 \\ \text { H } & -5.347953 & -1.581091 & 0.568824 \\ \text { H } & -3.954861 & -3.616998 & 0.833458 \\ \text { H } & -1.516839 & -3.484147 & 0.394167 \\ \text { H } & -0.495117 & -1.363729 & -0.298384 \\ \text { C } & -2.320117 & 1.729430 & -1.909685 \\ \text { C } & -2.355329 & 2.347435 & -0.540005 \\ \text { H } & -3.289238 & 2.389561 & 0.005991 \\ \text { H } & -1.677472 & 3.164874 & -0.340104 \\ \text { H } & -3.230028 & 1.305107 & -2.316891 \\ \text { H } & -1.632634 & 2.144840 & -2.636148 \\ \text { H } & 2.457754 & 0.756301 & 2.158069 \\ \text { H } & 4.702521 & -0.207953 & 1.757813 \\ \text { F } & 5.521081 & -1.203007 & -0.503661 \\ \text { H } & 3.643625 & -1.045530 & -2.298063 \\ \text { H } & 1.403067 & -0.083461 & -1.927181\end{array}$

Frequencies (Top 10 out of 90 )

1. $24.8157 \mathrm{~cm}-1$ (Symmetry: A)

2. $\quad 56.3199 \mathrm{~cm}-1$ (Symmetry: A)

3. $\quad 73.1143 \mathrm{~cm}-1$ (Symmetry: A)

4. $\quad 92.9923 \mathrm{~cm}-1$ (Symmetry: A)

5. $\quad 103.1879 \mathrm{~cm}-1$ (Symmetry: A)

6. $\quad 118.9110 \mathrm{~cm}-1$ (Symmetry: A)

7. $\quad 156.9054 \mathrm{~cm}-1$ (Symmetry: A)

8. $\quad 172.5633 \mathrm{~cm}-1$ (Symmetry: A)

9. $213.1404 \mathrm{~cm}-1$ (Symmetry: A)

10. $245.5052 \mathrm{~cm}-1$ (Symmetry: A)

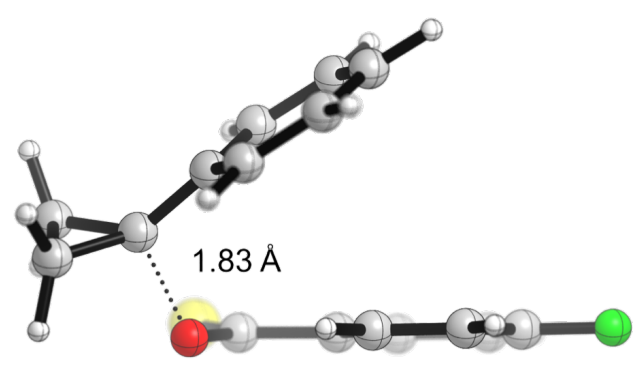

Charge -1

Multiplicity 2

Stoichiometry C16H13FOS(-1)

Electronic Energy (Eh) -1190.76689813

Number of Imaginary Frequencies 1

Mean of alpha and beta Electrons $\quad 71.5$

Molecular Geometry in Cartesian Coordinates

\begin{tabular}{llll}
\hline C & -1.219313 & -0.000260 & -1.096274 \\
C & -2.480851 & 0.567998 & -1.130814 \\
C & -3.483215 & -0.006755 & -0.375733 \\
C & -3.263829 & -1.121764 & 0.406423
\end{tabular}




$\begin{array}{lccc}\text { C } & -1.992478 & -1.672477 & 0.437481 \\ \text { C } & -0.940590 & -1.124184 & -0.306479 \\ \text { C } & 0.433027 & -1.684915 & -0.276759 \\ \text { S } & 0.867995 & -2.944069 & 0.808901 \\ \text { O } & 1.274063 & -1.149278 & -1.090668 \\ \text { C } & 2.452712 & 0.065114 & -0.393406 \\ \text { C } & 1.694523 & 1.148523 & 0.155815 \\ \text { C } & 1.439027 & 2.312706 & -0.588902 \\ \text { C } & 0.561033 & 3.271338 & -0.122706 \\ \text { C } & -0.096090 & 3.090085 & 1.093678 \\ \text { C } & 0.162330 & 1.953032 & 1.848971 \\ \text { C } & 1.052460 & 0.994186 & 1.395734 \\ \text { H } & 1.236305 & 0.096218 & 1.974407 \\ \text { H } & -0.347769 & 1.805225 & 2.794521 \\ \text { H } & -0.802953 & 3.832087 & 1.447461 \\ \text { H } & 0.370252 & 4.161285 & -0.713356 \\ \text { H } & 1.924531 & 2.437898 & -1.551277 \\ \text { C } & 3.638778 & 0.035804 & -1.256380 \\ \text { C } & 3.620128 & -0.675336 & 0.097081 \\ \text { H } & 4.192261 & -0.240589 & 0.911876 \\ \text { H } & 3.558884 & -1.757088 & 0.095446 \\ \text { H } & 4.231608 & 0.940018 & -1.359335 \\ \text { H } & 3.593259 & -0.591346 & -2.140971 \\ \text { H } & -1.789183 & -2.540341 & 1.052057 \\ \text { H } & -4.076387 & -1.545622 & 0.984552 \\ \text { F } & -4.725713 & 0.544415 & -0.403691 \\ \text { H } & -2.687560 & 1.447191 & -1.729117 \\ \text { H } & -0.418170 & 0.436079 & -1.676509 \\ & & & \end{array}$

Frequencies (Top 10 out of 90 )

1. $-790.9853 \mathrm{~cm}-1$ (Symmetry: A) *

2. $\quad 63.4610 \mathrm{~cm}-1$ (Symmetry: A)

3. $\quad 72.1982 \mathrm{~cm}-1$ (Symmetry: A)

4. $92.0594 \mathrm{~cm}-1$ (Symmetry: A)

5. $\quad 105.4938 \mathrm{~cm}-1$ (Symmetry: A)

6. $\quad 111.9516 \mathrm{~cm}-1$ (Symmetry: A)

7. $\quad 152.9136 \mathrm{~cm}-1$ (Symmetry: A)

8. $\quad 180.1198 \mathrm{~cm}-1$ (Symmetry: A)

9. $\quad 183.4795 \mathrm{~cm}-1$ (Symmetry: A)

10. $213.4082 \mathrm{~cm}-1$ (Symmetry: A)

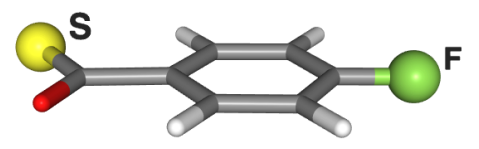

Charge -1

Multiplicity 1

Stoichiometry C7H4FOS(-1)

Electronic Energy (Eh) -842.521121919

Number of Imaginary Frequencies 0

Mean of alpha and beta Electrons $\quad 40$

Molecular Geometry in Cartesian Coordinates

\begin{tabular}{llll}
\hline C & -0.513182 & 1.300177 & -0.000004 \\
C & -1.890926 & 1.141735 & -0.000003
\end{tabular}




$\begin{array}{lrrr}\text { C } & -2.398373 & -0.140803 & 0.000000 \\ \text { C } & -1.587508 & -1.256633 & 0.000002 \\ \text { C } & -0.212359 & -1.072838 & 0.000002 \\ \text { C } & 0.344762 & 0.202628 & -0.000002 \\ \text { C } & 1.850793 & 0.463065 & 0.000001 \\ \text { S } & 2.922470 & -0.891758 & -0.000002 \\ \text { O } & 2.198017 & 1.641190 & 0.000006 \\ \text { H } & 0.457216 & -1.924119 & 0.000002 \\ \text { H } & -2.028482 & -2.246109 & 0.000004 \\ \text { F } & -3.743843 & -0.310696 & 0.000001 \\ \text { H } & -2.563211 & 1.991227 & -0.000004 \\ \text { H } & -0.073828 & 2.289895 & -0.000003\end{array}$

Frequencies (Top 10 out of 36 )

1. $\quad 75.9492 \mathrm{~cm}-1$ (Symmetry: A)

2. $\quad 120.8453 \mathrm{~cm}-1$ (Symmetry: A)

3. $\quad 191.7927 \mathrm{~cm}-1$ (Symmetry: A)

4. $\quad 303.4773 \mathrm{~cm}-1$ (Symmetry: A)

5. $310.9199 \mathrm{~cm}-1$ (Symmetry: A)

6. $\quad 398.9774 \mathrm{~cm}-1$ (Symmetry: A)

7. $441.8967 \mathrm{~cm}-1$ (Symmetry: A)

8. $\quad 463.3094 \mathrm{~cm}-1$ (Symmetry: A)

9. $\quad 510.2775 \mathrm{~cm}-1$ (Symmetry: A)

10. $529.2753 \mathrm{~cm}-1$ (Symmetry: A) 


\section{H. References}

${ }^{1}$ Côté, A.; Charette, A. B. J. Am. Chem. Soc. 2008, 130, 2771-2773.

${ }^{2}$ Hixson, S. S.; Franke, L. A. J. Org. Chem. 1988, 53, 2706-2711.

${ }^{3}$ Krasovskiy, A.; Knochel, P. Synthesis 2006, 890-891.

${ }^{4}$ Mills, L. R.; Graham, J. M.; Patel, P.; Rousseaux, S. A. L. J. Am. Chem. Soc. 2019, 141, 19257-19262.

${ }^{5}$ Keylor, M. H.; Niemeyer, Z. L.; Sigman, M. S.; Tan, Kian. L. J. Am. Chem. Soc. 2017, 139, 10613-10616.

${ }^{6}$ Salaun, J. J. Org. Chem. 1978, 43, 2809-2815.

${ }^{7}$ Lackner, G. L.; Quasdorf, K. W.; Overman, L. E. J. Am. Chem. Soc. 2013, 41, 15342-15345.

${ }^{8}$ Mills, L. R.; Zhou, C.; Fung, E.; Rousseaux, S. A. L. Org. Lett. 2019, 21, 8805-8809.

${ }^{9}$ Nomura, K.; Matsubara, S. Chem. Lett. 2007, 36, 164-165.

${ }^{10}$ Jia, K.; Zhang, F.; Huang, H.; Chen, Y. J. Am. Chem. Soc. 2016, 138, 1514-1517.

${ }^{11}$ Nomura, K.; Matsubara, S. Chem. Asian J. 2010, 5, 147-152.

12 Jiao, J.; Nguyen, L. X.; Patterson, D. R.; Floewrs, R. A. Org. Lett. 2007, 9, 1323-1326.

${ }^{13}$ Pramanik, S.; Rej, S.; Kando, S.; Tsurugi, H.; Mashima, K. J. Org. Chem. 2018, 83, 24092417.

${ }^{14}$ Vita, M. V.; Waser, J. Org. Lett. 2013, 15, 3246-3249.

${ }^{15}$ Fujita, K.; Yorimitsu, H.; Shinokubo, H.; Matsubara, S.; Oshima, K. J. Am. Chem. Soc. 2001, $123,12115-12116$.

${ }^{16}$ Wei, Y.; Xue, D.; Lei, Q.; Wang, C.; Xiao, J. Green Chem. 2013, 15, 629-634.

${ }^{17}$ Li, H.; Breen, C. P.; Seo, H.; Jamison, T. F.; Fang, Y.-Q.; Bio, M. M. Org. Lett. 2018, 20, 1338-1341.

${ }^{18}$ Puthiaraj, P.; Suresh, P.; Pitchumani, K. Green Chem. 2014, 16, 2865-2875.

${ }^{19}$ Noji, M.; Ohno, T.; Fuji, K.; Futaba, N.; Tajima, H.; Ishii, K. J. Org. Chem. 2003, 68, 93409347.

${ }^{20}$ Gaussian '16, Revision C.01, Frisch, M. J.; Trucks, G. W.; Schlegel, H. B.; Scuseria, G. E.; Robb, M. A.; Cheeseman, J. R.; Scalmani, G.; Barone, V.; Petersson, G. A.; Nakatsuji, H.; Li, X.; Caricato, M.; Marenich, A. V.; Bloino, J.; Janesko, B. G.; Gomperts, R.; Mennucci, B.; Hratchian, H. P.; Ortiz, J. V.; Izmaylov, A. F.; Sonnenberg, J. L.; Williams-Young, D.; Ding, F.; Lipparini, F.; Egidi, F.; Goings, J.; Peng, B.; Petrone, A.; Henderson, T.; Ranasinghe, D.; Zakrzewski, V. G.; Gao, J.; Rega, N.; Zheng, G.; Liang, W.; Hada, M.; Ehara, M.; Toyota, K.; Fukuda, R.; Hasegawa, J.; Ishida, M.; Nakajima, T.; Honda, Y.; Kitao, O.; Nakai, H.; Vreven, T.; Throssell, K.; Montgomery, J. A., Jr.; Peralta, J. E.; Ogliaro, F.; Bearpark, M. J.; Heyd, J. J.; Brothers, E. N.; Kudin, K. N.; Staroverov, V. N.; Keith, T. A.; Kobayashi, R.; Normand, J.; Raghavachari, K.; Rendell, A. P.; Burant, J. C.; Iyengar, S. S.; Tomasi, J.; Cossi, M.; Millam, J. M.; Klene, M.; Adamo, C.; Cammi, R.; Ochterski, J. W.; Martin, R. L.; Morokuma, K.; Farkas, O.; Foresman, J. B.; Fox, D. J. Gaussian, Inc., Wallingford CT, 2016.

${ }^{21}$ (a) O'Boyle, N. M.; Banck, M.; James, C. A.; Morley, C.; Vandermeersch, T.; Hutchison, G. R. "Open Babel: An open chemical toolbox." J. Cheminf., 2011, 3, 33. (b) The Open Babel Package, version 2.3.1 http://openbabel.org (accessed Feb 2019).

${ }^{22} x t b$ can be freely obtained at: https://github.com/grimme-lab/xtb. Bannwarth, C.; Ehlert, S.; Grimme, S. GFN2-xTB - An Accurate and Broadly Parametrized Self-Consistent Tight-Binding Quantum Chemical Method with Multipole Electrostatics and Density-Dependent Dispersion Contributions. J. Chem. Theory Comput. 2019, 15, 1652. 
${ }^{23}$ Grimme, S. Exploration of Chemical Compound, Conformer, and Reaction Space with MetaDynamics Simulations Based on Tight-Binding Quantum Chemical Calculations. J. Chem. Theory Comput. 2019, 15 (5), 2847.

${ }^{24}$ Marenich, A. V.; Cramer, C. J.; Truhlar, D. G. "Universal solvation model based on solute electron density and a continuum model of the solvent defined by the bulk dielectric constant and atomic surface tensions," J. Phys. Chem. B, 2009, 113, 6378.

${ }^{25}$ Chai, J.-D.; Head-Gordon, M. "Long-range corrected hybrid density functionals with damped atom-atom dispersion corrections," Phys. Chem. Chem. Phys., 2008, 10, 6615.

${ }^{26}$ (a) Grimme, S.; Antony, J.; Ehrlich, S.; Krieg, H. J. Chem. Phys., 2010, 132, 154104. (b) Grimme, S.; Ehrlich, S.; Goerigk, L. J. Comp. Chem., 2011, 32, 1456.

${ }^{27}$ GoodVibes can be freely obtained at: https://github.com/bobbypaton/GoodVibes. Luchini, G.; Alegre-Requena, J. V.; Funes-Ardoiz, I.; Paton, R. S. GoodVibes: Automated Thermochemistry for Heterogeneous Computational Chemistry Data. F1000Research, 2020, 9, 291.

${ }^{28}$ (a) Weinhold, F.; Landis, C. R.; Glendening, E. D. International Reviews in Physical Chemistry 2016, 35, 1. Reed, A. E.; Weinhold, F. J. Chem. Phys., 1985, 83, 1736. (b) Reed, A. E.; Weinhold, F. Isr. J. Chem., 1991, 31, 277. (c) Reed, A. E.; Curtiss, L. A.; Weinhold, F. Chem. Rev., 1988, 88, 899. (d) Weinhold F. in Schleyer P.v.R. Encyclopedia of Computational Chemistry: Wiley: NewYork, 1998, 3, 1792. Selected recent applications of NBO analysis towards analysis of organic structure and reactivity: a) Podlech, J. J. Phys. Chem. A 2010, 114, 8480. b) Freitas, M. P. J. Org. Chem. 2012, 77, 7607. c) Greenway, K. T.; Bischoff, A. G.; Pinto, B. M. J. Org. Chem. 2012, 77, 9221. d) Juaristi, E.; Notario, R. J. Org. Chem. 2015, 80, 2879. e) Gomes, G. P.; Vil', V.; Terent'ev, A.; Alabugin, I. V. Chem. Sci. 2015, 6, 6783. f) Vidhani, D.; Krafft, M.; Alabugin, I. V. J. Am. Chem. Soc., 2016, 138, 2769. g) V. A. Vil', G. dos Passos Gomes, O. V. Bityukov, K. A. Lyssenko, G. I. Nikishin, I. V. Alabugin, A. O. Terent'ev, Angew. Chem. Int. Ed., 2018, 57, 3372.

${ }^{29}$ CYLview, 1.0b; Legault, C. Y., Université de Sherbrooke, 2009 (http://www.cylview.org) 30 ESIgen can be freely obtained at: https://github.com/insilichem/esigen. J. Chem. Inf. Model., 2018, 58 (3), 561. 